Palladium-Catalyzed Carboannulation of Propargylic Carbonates and Nucleophiles to 2-Substituted Indenes

Li-Na Guo, ${ }^{a}$ Xin-Hua Duan, ${ }^{a}$ Hai-Peng Bi, ${ }^{a}$ Xue-Yuan Liu,,${ }^{a}$ Yong-Min Liang ${ }^{*}$ ab

${ }^{a}$ State Key Laboratory of Applied Organic Chemistry, Lanzhou University.

${ }^{\mathrm{b}}$ State Key Laboratory of Solid Lubrication, Lanzhou Institute of Chemical Physics, Chinese Academy of Science

Lanzhou 730000, P.R. China. Fax: +86-931-8912582; Tel: +86-931-8912593

liangym@1zu.edu.cn

Table of Contents

1. General Remarks

S2

2. Typical experimental procedure for starting materials synthesis $\mathbf{S 2 - S 3}$

3. References

S2

4. Characterization data of compounds 1,11 and 4

S2-S3

5. Typical experimental procedure for indenes 3 synthesis

S3

6. Experimental procedure for the cyclization reaction of 4

S3

7. Characterization data of compounds 3a-3o

S4-S8

8. Typical experimental procedure for indenes 6 synthesis

S8

9. Characterization data of compounds 6a-6g

S8-S10

10. ${ }^{1} \mathrm{HNMR}$ and ${ }^{13} \mathrm{CNMR}$ spectra for compounds 1,11 and $4 \quad$ S11-S16

11. ${ }^{1}$ HNMR and ${ }^{13}$ CNMR spectra for compounds 3a-3o S17-S46

12. ${ }^{1} \mathrm{HNMR}$ and ${ }^{13}$ CNMR spectra for compounds $6 \mathrm{a}-6 \mathrm{~g} \quad \mathrm{S47-S60}$ 


\section{General Remarks:}

Column chromatography was carried out on silica gel. ${ }^{1} \mathrm{H}$ NMR spectra were recorded on $300 \mathrm{MHz}$ or $400 \mathrm{MHz}$ in $\mathrm{CDCl}_{3}$ and ${ }^{13} \mathrm{C}$ NMR spectra were recorded on $75 \mathrm{MHz}$ or $100 \mathrm{MHz}$ in $\mathrm{CDCl}_{3}$ using TMS as internal standard. IR spectra were recorded on a FT-IR spectrometer and only major peaks are reported in $\mathrm{cm}^{-1}$. Melting points were determined on a microscopic apparatus and were uncorrected. All new compounds were further characterized by element analysis; copies of their ${ }^{1} \mathrm{H}$ NMR and ${ }^{13} \mathrm{C}$ NMR spectra are provided. Unless otherwise stated, all nucleophiles were purchased from commercial suppliers and used without further purification.

\section{Starting Materials:}

Diethyl (2-iodophenyl)malonate and ethyl (2-iodophenyl)(phenylsulfonyl)acetate were prepared according to the literature. ${ }^{1}$

\section{References}

(1) Zhang, D.; Yum, E. K.; Liu, Z.; Larock, R. C. Org. Lett. 2005, 7, 4963.

\section{Typical procedure for the preparation of propargylic alcohol}

To a solution of diethyl (2-iodophenyl)malonate $(0.72 \mathrm{~g}, 2.0 \mathrm{mmol})$ and prop-2-yn-1-ol $(0.13 \mathrm{~g}, 2.4 \mathrm{mmol})$ in $\mathrm{Et}_{3} \mathrm{~N}(8.0 \mathrm{~mL})$ was added $\mathrm{PdCl}_{2}\left(\mathrm{PPh}_{3}\right)_{2}(70 \mathrm{mg}, 5 \mathrm{~mol} \%)$. The mixture was stirred for $5 \mathrm{~min}$ and $\mathrm{CuI} \mathrm{(38} \mathrm{mg}, 10 \mathrm{~mol} \%$ ) was added. The resulting mixture was then stirring under an argon atmosphere at room temperature for $12 \mathrm{~h}$. The ammonium salt was removed by filtration. The solvent was removed under reduced pressure and the residue was purified by column chromatography on silica gel to afford diethyl 2-(2-(3-hydroxyprop-1-ynyl) phenyl)malonate 0.41g (70 \%) as an oil; ${ }^{1} \mathrm{H}$ NMR $\left(300 \mathrm{MHz}, \mathrm{CDCl}_{3}\right) \delta$ 7.47-7.44 (m, 2H), 7.38-7.24 (m, 2H), $5.25(\mathrm{~s}, 1 \mathrm{H}), 4.48(\mathrm{~s}, 2 \mathrm{H})$, 4.27-4.18 (m, 4H), $2.75(\mathrm{~s}, 1 \mathrm{H}), 1.28-1.22(\mathrm{~m}, 6 \mathrm{H}) ;{ }^{13} \mathrm{C} \mathrm{NMR}\left(75 \mathrm{MHz}, \mathrm{CDCl}_{3}\right) \delta$ $168.0,134.8,132.1,128.7,128.6,127.8,123.0,92.7,82.9,61.9,55.9,51.3,13.9$; IR $\left(\mathrm{KBr}, \mathrm{cm}^{-1}\right)$ 3452, 2984, 1733, 1486, 1306, 1219, 1151, 1031.

Typical procedure for the preparation of propargylic carbonates 1, 11 and 4. To a solution of propargylic alcohol $(2.0 \mathrm{mmol})$, pyridine $(0.63 \mathrm{~g}, 8.0 \mathrm{mmol})$, and DMAP (44.8 mg, $0.4 \mathrm{mmol})$ in $\mathrm{CH}_{2} \mathrm{Cl}_{2}(10 \mathrm{~mL})$ was added at $0{ }^{\circ} \mathrm{C}$ methyl chloroformate $(0.75$ $\mathrm{g}, 8.0 \mathrm{mmol}$ ). After stirring for $2 \mathrm{~h}$ at room temperature, the reaction mixture was diluted with $\mathrm{CH}_{2} \mathrm{Cl}_{2}$. The $\mathrm{CH}_{2} \mathrm{Cl}_{2}$ solution was washed with a saturated aqueous copper sulfate solution, water, dried over anhydrous sodium sulfate, and concentrated. The residue was purified by column chromatography on silica gel to afford the corresponding propargylic carbonates.

3-(2-(Di(ethoxycarbonyl)methyl)phenyl)prop-2-ynyl methyl carbonate (1): The 1 was prepared by the above method, But employing diethyl 2-(2-(3-hydroxyprop-1-ynyl)phenyl)malonate $(0.58 \mathrm{~g}, 2.0 \mathrm{mmol})$ and methyl chloroformate afforded $10.61 \mathrm{~g}(87 \%)$ as a solid: mp 59-61 ${ }^{\circ} \mathrm{C} ;{ }^{1} \mathrm{H}$ NMR $(300 \mathrm{MHz}$, $\left.\mathrm{CDCl}_{3}\right) \delta 7.50-7.48(\mathrm{~d}, J=7.5 \mathrm{~Hz}, 2 \mathrm{H}), 7.41-7.35(\mathrm{~m}, 1 \mathrm{H}), 7.32-7.26(\mathrm{~m}, 1 \mathrm{H}), 5.24(\mathrm{~s}$, $1 \mathrm{H}), 4.99(\mathrm{~s}, 2 \mathrm{H}), 4.29-4.18(\mathrm{~m}, 4 \mathrm{H}), 3.84(\mathrm{~s}, 3 \mathrm{H}), 1.30-1.25(\mathrm{t}, J=6.9 \mathrm{~Hz}, 6 \mathrm{H}) ;{ }^{13} \mathrm{C}$ NMR $\left(75 \mathrm{MHz}, \mathrm{CDCl}_{3}\right) \delta 167.9,155.1,135.1,132.5,129.2,128.6,127.9,122.4,87.2$, 84.6, 61.8, 56.0, 55.5, 55.1, 13.9; IR (KBr, cm $\left.{ }^{-1}\right)$ 3478, 2987, 1753, 1445, 1267, 1027; Anal.Calcd for $\mathrm{C}_{18} \mathrm{H}_{20} \mathrm{O}_{7}$ : C 62.06; H 5.79. Found: C 61.82; H 6.01. 
carbonate (11): The 11 was prepared by the same method, But employing ethyl 2-(2-(3-hydroxyprop-1-ynyl)phenyl)-2-(phenylsulfonyl)acetate $(0.72 \mathrm{~g}, 2.0 \mathrm{mmol})$ and methyl chloroformate afforded $110.69 \mathrm{~g}(80 \%)$ as a solid: $\mathrm{mp} 84-86{ }^{\circ} \mathrm{C} ;{ }^{1} \mathrm{H}$ NMR $(300$ $\left.\mathrm{MHz}, \mathrm{CDCl}_{3}\right) \delta$ 7.98-7.96 (d, J=7.5 Hz, $\left.1 \mathrm{H}\right), 7.70-7.68(\mathrm{~m}, 2 \mathrm{H}), 7.63-7.58(\mathrm{~m}, 1 \mathrm{H})$, 7.47-7.28 (m, 5H), $5.85(\mathrm{~s}, 1 \mathrm{H}), 4.87(\mathrm{~s}, 2 \mathrm{H}), 4.30-4.17(\mathrm{~m}, 2 \mathrm{H}), 3.87(\mathrm{~s}, 3 \mathrm{H})$, $1.27-1.22(\mathrm{t}, J=7.2 \mathrm{~Hz}, 3 \mathrm{H}) ;{ }^{13} \mathrm{C}$ NMR $\left(75 \mathrm{MHz}, \mathrm{CDCl}_{3}\right) \delta 164.1,155.1,137.4,134.0$, 132.3, 130.0, 129.7, 129.4, 129.2, 129.1, 128.6, 123.7, 87.3, 83.8, 71.1, 62.6, 55.7, 55.2, 13.8; IR (KBr, $\left.\mathrm{cm}^{-1}\right)$ 3466, 2959, 1746, 1446, 1264, 1151, 1084, 1022; Anal.Calcd for $\mathrm{C}_{21} \mathrm{H}_{20} \mathrm{O}_{7} \mathrm{~S}: \mathrm{C} 60.57 ; \mathrm{H} 4.84$. Found: $\mathrm{C} 60.55 ; \mathrm{H} 4.69$.

2-[2-(3-Phenoxycarbonyloxy-prop-1-ynyl)-phenyl]-malonic acid diethyl ester (4): The 4 was prepared by the above method, But employing diethyl 2-(2-(3-hydroxyprop-1-ynyl)phenyl)malonate $(0.58 \mathrm{~g}, 2.0 \mathrm{mmol})$ and phenyl chloroformate afforded $40.70 \mathrm{~g}(85 \%)$ as an oil: ${ }^{1} \mathrm{H}$ NMR $\left(300 \mathrm{MHz}, \mathrm{CDCl}_{3}\right) \delta$ 7.52-7.50 (d, $J=7.8 \mathrm{~Hz}, 2 \mathrm{H}), 7.42-7.36(\mathrm{~m}, 3 \mathrm{H}), 7.33-7.20$ (m, 4H), 5.26 (s, 1H), 5.09 (s, 2H), 4.27-4.19 (m, 4H), 1.28-1.23 (m, 6H); ${ }^{13} \mathrm{C} \mathrm{NMR}\left(75 \mathrm{MHz}, \mathrm{CDCl}_{3}\right) \delta 167.9$, 153.1, 151.0, 135.2, 132.5, 129.5, 129.3, 128.7, 127.9, 126.2, 122.3, 120.9, 86.8, 85.1, 61.9, 56.6, 55.6, 14.0; IR (neat, $\mathrm{cm}^{-1}$ ) 3454, 2984, 1763, 1734, 1490, 1239, 1213, 1029; Anal.Calcd for $\mathrm{C}_{23} \mathrm{H}_{22} \mathrm{O}_{7}$ : C 67.31; H 5.40. Found: C 67.13; H 5.27.

General Procedure for the Preparation of 2-Substituted Indenes 3.

A mixture of 3-(2-(di(ethoxycarbonyl)methyl)phenyl)prop-2-ynyl methyl carbonate (1; $69.6 \mathrm{mg}, 0.20 \mathrm{mmol})$, phenols $(0.24 \mathrm{mmol}), \mathrm{Pd}\left(\mathrm{PPh}_{3}\right)_{4}(11.5 \mathrm{mg}, 5 \mathrm{~mol} \%)$, and THF (2.0 mL) was placed under argon atmosphere in a $25 \mathrm{~mL}$ flask. The resulting mixture was then heated under an argon atmosphere at $80{ }^{\circ} \mathrm{C}$. When the reaction was considered complete as determined by TLC analysis, the reaction mixture was allowed to cool to room temperature. The reaction mixture was concentrated under reduced pressure and the residue was purified by chromatography on silica gel to afford the corresponding 2-substituted indenes 3 .

The cyclization of 2-[2-(3-Phenoxycarbonyloxy-prop-1-ynyl)-phenyl]-malonic acid diethyl ester (4) to 2-substituted indene 3a.

A mixture of $4(82.0 \mathrm{mg}, 0.20 \mathrm{mmol}), \mathrm{Pd}\left(\mathrm{PPh}_{3}\right)_{4}(11.5 \mathrm{mg}, 5 \mathrm{~mol} \%)$, and THF $(2.0$ $\mathrm{mL}$ ) was placed under argon atmosphere in a $25 \mathrm{~mL}$ flask. The resulting mixture was then heated under an argon atmosphere at $80{ }^{\circ} \mathrm{C}$. When the reaction was considered complete as determined by TLC analysis, the reaction mixture was allowed to cool to room temperature. The reaction mixture was concentrated under reduced pressure and the residue was purified by chromatography on silica gel to afford the product $\mathbf{3 a} 60.8$ $\mathrm{mg}(83 \%)$.

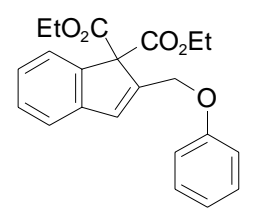

3a: The reaction mixture was chromatographed using 10:1 hexanes/EtOAc to afford $70.0 \mathrm{mg}(96 \%)$ of the indicated compound as an oil: ${ }^{1} \mathrm{H}$ NMR $\left(400 \mathrm{MHz}, \mathrm{CDCl}_{3}\right) \delta$ 7.67-7.65 (d, $J=7.2 \mathrm{~Hz}, 1 \mathrm{H}), 7.34-7.22(\mathrm{~m}, 5 \mathrm{H}), 7.02-7.01(\mathrm{~d}, J=7.6 \mathrm{~Hz}, 2 \mathrm{H})$, 
6.97-6.94 (m, 2H), $5.10(\mathrm{~s}, 2 \mathrm{H}), 4.26-4.21(\mathrm{q}, J=7.2 \mathrm{~Hz}, 4 \mathrm{H}), 1.27-1.23(\mathrm{~m}, 6 \mathrm{H}) ;{ }^{13} \mathrm{C}$ NMR $\left(100 \mathrm{MHz}, \mathrm{CDCl}_{3}\right) \delta 167.6,158.7,143.3,141.7,140.6,132.6,129.4,128.8$, 126.0, 125.2, 121.4, 120.9, 114.8, 70.7, 65.4, 62.3, 13.9; IR (neat, $\mathrm{cm}^{-1}$ ) 3447, 2982, 1732, 1495, 1242, 1047; Anal.Calcd for $\mathrm{C}_{22} \mathrm{H}_{22} \mathrm{O}_{5}$ : C 72.12; H 6.05. Found: C 72.02; H 5.94 .

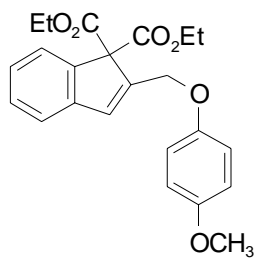

3b: The reaction mixture was chromatographed using 10:1 hexanes/EtOAc to afford $73.6 \mathrm{mg}(93 \%)$ of the indicated compound as an oil: ${ }^{1} \mathrm{H}$ NMR $\left(400 \mathrm{MHz}, \mathrm{CDCl}_{3}\right) \delta$ 7.67-7.65 (dd, $J=7.6,0.8 \mathrm{~Hz}, 1 \mathrm{H}), 7.33-7.22(\mathrm{~m}, 3 \mathrm{H}), 6.96-6.93(\mathrm{~m}, 3 \mathrm{H}), 6.84-6.82$ $(\mathrm{m}, 2 \mathrm{H}), 5.05(\mathrm{~s}, 2 \mathrm{H}), 4.25-4.20(\mathrm{~m}, 4 \mathrm{H}), 3.75(\mathrm{~s}, 3 \mathrm{H}), 1.26-1.23(\mathrm{~m}, 6 \mathrm{H}) ;{ }^{13} \mathrm{C} \mathrm{NMR}$ $\left(100 \mathrm{MHz}, \mathrm{CDCl}_{3}\right) \delta 167.6,153.9,152.8,143.2,141.9,140.5,132.5,128.7,126.0$, 125.1, 121.4, 115.6, 114.6, 70.5, 66.0, 62.2, 55.6, 13.9; IR (neat, $\mathrm{cm}^{-1}$ ) 3440, 2983, 1732, 1507, 1231, 1043; Anal.Calcd for $\mathrm{C}_{23} \mathrm{H}_{24} \mathrm{O}_{6}$ : C 69.68; H 6.10. Found: C 69.96; H 5.95 .

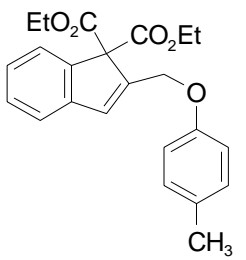

3c: The reaction mixture was chromatographed using 10:1 hexanes/EtOAc to afford $70.1 \mathrm{mg}(92 \%)$ of the indicated compound as an oil: ${ }^{1} \mathrm{H}$ NMR $\left(400 \mathrm{MHz}, \mathrm{CDCl}_{3}\right) \delta$ 7.67-7.65 (dd, $J=7.6,0.8 \mathrm{~Hz}, 1 \mathrm{H}), 7.32-7.21(\mathrm{~m}, 3 \mathrm{H}), 7.09-7.06(\mathrm{~m}, 2 \mathrm{H}), 6.94-6.90$ $(\mathrm{m}, 3 \mathrm{H}), 5.08(\mathrm{~s}, 2 \mathrm{H}), 4.25-4.20(\mathrm{~m}, 4 \mathrm{H}), 2.28(\mathrm{~s}, 3 \mathrm{H}), 1.26-1.23(\mathrm{~m}, 6 \mathrm{H}) ;{ }^{13} \mathrm{C} \mathrm{NMR}$ $\left(100 \mathrm{MHz}, \mathrm{CDCl}_{3}\right) \delta 167.6,156.5,143.3,141.8,140.5,132.5,130.0,129.8,128.7$, 125.9, 125.1, 121.4, 114.5, 70.6, 65.4, 62.2, 20.4, 13.9; IR (neat, $\mathrm{cm}^{-1}$ ) 3463, 2982, 1730, 1511, 1242, 1050; Anal.Calcd for $\mathrm{C}_{23} \mathrm{H}_{24} \mathrm{O}_{5}$ : C 72.61; H 6.36. Found: C 72.48; H 6.18 .

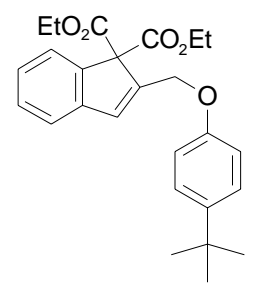

3d: The reaction mixture was chromatographed using 10:1 hexanes/EtOAc to afford $76 \mathrm{mg}(90 \%)$ of the indicated compound as an oil: ${ }^{1} \mathrm{H}$ NMR $\left(400 \mathrm{MHz}, \mathrm{CDCl}_{3}\right) \delta$ 7.67-7.65 (d, $J=8.0 \mathrm{~Hz}, 1 \mathrm{H}), 7.33-7.21(\mathrm{~m}, 5 \mathrm{H}), 6.97-6.94(\mathrm{~m}, 3 \mathrm{H}), 5.09(\mathrm{~s}, 2 \mathrm{H})$, 4.25-4.20 (q, $J=7.2 \mathrm{~Hz}, 4 \mathrm{H}), 1.30(\mathrm{~s}, 9 \mathrm{H}), 1.28-1.23(\mathrm{~m}, 6 \mathrm{H}) ;{ }^{13} \mathrm{C}$ NMR $(100 \mathrm{MHz}$, $\left.\mathrm{CDCl}_{3}\right) \delta 167.6,156.4,143.5,143.3,141.9,140.6,132.5,128.7,126.2,125.9,125.1$, 121.4, 114.2, 70.6, 65.4, 62.2, 34.0, 31.5, 13.9; IR (neat, $\mathrm{cm}^{-1}$ ) 3466, 2962, 1734, 1512, 1245, 1049; Anal.Calcd for $\mathrm{C}_{26} \mathrm{H}_{30} \mathrm{O}_{5}$ : C 73.91; H 7.16. Found: C 73.88; H 7.04. 


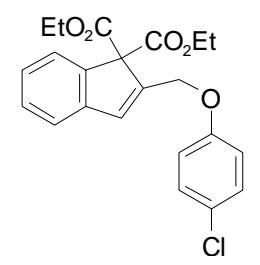

3e: The reaction mixture was chromatographed using 10:1 hexanes/EtOAc to afford $72.8 \mathrm{mg}(91 \%)$ of the indicated compound as an oil: ${ }^{1} \mathrm{H}$ NMR $\left(400 \mathrm{MHz}, \mathrm{CDCl}_{3}\right) \delta$ 7.68-7.66 (d, $J=7.6 \mathrm{~Hz}, 1 \mathrm{H}), 7.33-7.22(\mathrm{~m}, 5 \mathrm{H}), 6.97-6.91(\mathrm{~m}, 3 \mathrm{H}), 5.08(\mathrm{~s}, 2 \mathrm{H})$, 4.27-4.20 (m, 4H), 1.28-1.21 (m, 6H); $\left.{ }^{13} \mathrm{C} \mathrm{NMR} \mathrm{(100} \mathrm{MHz,} \mathrm{CDCl}_{3}\right) \delta 167.5,157.3$, 143.1, 141.1, 140.5, 132.7, 129.3, 128.8, 126.1, 125.8, 125.2, 121.5, 116.1, 70.6, 65.8, 62.3, 13.9; IR (neat, $\mathrm{cm}^{-1}$ ) 2982, 1732, 1491, 1243, 1049; Anal.Calcd for $\mathrm{C}_{22} \mathrm{H}_{21} \mathrm{ClO}_{5}$ : C 65.92; H 5.28. Found: C 65.68; H 5.34.

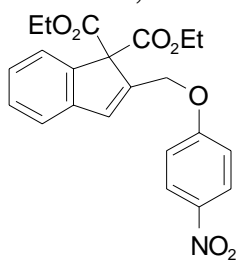

3f: The reaction mixture was chromatographed using 10:1 hexanes/EtOAc to afford $62.5 \mathrm{mg}(76 \%)$ of the indicated compound as an oil: ${ }^{1} \mathrm{H}$ NMR $\left(400 \mathrm{MHz}, \mathrm{CDCl}_{3}\right) \delta$ 8.21-8.19 (m, 2H), 7.69-7.67 (m, 1H), 7.35-7.32 (m, 1H), 7.29-7.25 (m, 2H), 7.12-7.10 $(\mathrm{dd}, J=7.2,1.6 \mathrm{~Hz}, 2 \mathrm{H}), 6.90(\mathrm{~s}, 1 \mathrm{H}), 5.22(\mathrm{~s}, 2 \mathrm{H}), 4.28-4.23(\mathrm{~m}, 4 \mathrm{H}), 1.29-1.26(\mathrm{~m}$, $6 \mathrm{H}) ;{ }^{13} \mathrm{C}$ NMR $\left(100 \mathrm{MHz}, \mathrm{CDCl}_{3}\right) \delta 167.3,163.6,142.7,141.7,140.3,139.9,133.1$, 128.9, 126.3, 125.9, 125.3, 121.6, 114.8, 70.5, 66.2, 62.5, 13.9; IR (neat, $\mathrm{cm}^{-1}$ ) 3445, 2983, 1731, 1593, 1515, 1258, 1049; Anal.Calcd for $\mathrm{C}_{22} \mathrm{H}_{21} \mathrm{NO}_{7}$ : C 64.23; H 5.14; N 3.40. Found: C 63.99; H 5.08; N 3.21.

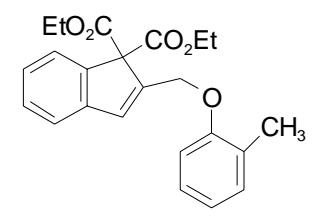

3g: The reaction mixture was chromatographed using 10:1 hexanes/EtOAc to afford $60.8 \mathrm{mg}(80 \%)$ of the indicated compound as a solid: $\mathrm{mp}: 38-40{ }^{\circ} \mathrm{C} ;{ }^{1} \mathrm{H}$ NMR (400 $\left.\mathrm{MHz}, \mathrm{CDCl}_{3}\right) \delta$ 7.68-7.66 (d, $\left.J=7.2 \mathrm{~Hz}, 1 \mathrm{H}\right), 7.34-7.22(\mathrm{~m}, 3 \mathrm{H}), 7.17-7.12(\mathrm{~m}, 2 \mathrm{H})$, 6.96-6.94 (m, 2H), 6.89-6.85 (t, J=7.2 Hz, 1H), 5.10 (s, 2H), 4.26-4.20 (m, 4H), 2.32 (s, 3H), 1.27-1.23 (m, 6H); ${ }^{13} \mathrm{C}$ NMR $\left(100 \mathrm{MHz}, \mathrm{CDCl}_{3}\right) \delta 167.6,156.7,143.3,141.9$, 140.5, 132.1, 130.6, 128.8, 126.8, 126.7, 125.9, 125.1, 121.4, 120.5, 111.3, 70.6, 65.3, 62.3, 16.3, 13.9; IR (KBr, cm $\left.{ }^{-1}\right) 3427,2982,1733,1495$, 1243, 1051; Anal.Calcd for $\mathrm{C}_{23} \mathrm{H}_{24} \mathrm{O}_{5}$ : C 72.61; H 6.36. Found: C 72.85; H 6.19.

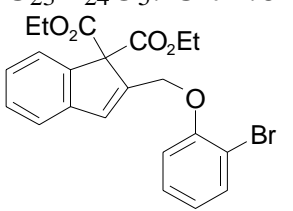

3h: The reaction mixture was chromatographed using 10:1 hexanes/EtOAc to afford $74.6 \mathrm{mg}(84 \%)$ of the indicated compound as an oil: ${ }^{1} \mathrm{H}$ NMR $\left(400 \mathrm{MHz}, \mathrm{CDCl}_{3}\right) \delta$ 7.68-7.66 (d, $J=7.6 \mathrm{~Hz}, 1 \mathrm{H}), 7.57-7.55(\mathrm{dd}, J=8.0,1.6 \mathrm{~Hz}, 1 \mathrm{H}), 7.34-7.21(\mathrm{~m}, 4 \mathrm{H})$, 
7.08-7.02 (m, 2H), 6.86-6.82 (m, 1H), $5.18(\mathrm{~s}, 2 \mathrm{H}), 4.27-4.22(\mathrm{~m}, 4 \mathrm{H}), 1.28-1.24(\mathrm{t}, J=$ $7.2 \mathrm{~Hz}, 6 \mathrm{H}) ;{ }^{13} \mathrm{C}$ NMR $\left(100 \mathrm{MHz}, \mathrm{CDCl}_{3}\right) \delta 167.5,154.9,143.2,140.6,140.4,133.3$, $132.7,128.8,128.5,126.0,125.2,122.0,121.5,113.5,112.0,70.6,66.3,62.4,13.9$; IR (neat, $\mathrm{cm}^{-1}$ ) 3447, 2982, 1732, 1478, 1248, 1051; Anal.Calcd for $\mathrm{C}_{22} \mathrm{H}_{21} \mathrm{BrO}_{5}$ : C 59.34; H 4.75. Found: C 59.23; H 4.69.

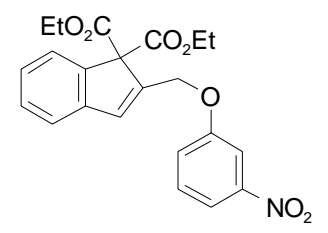

3i: The reaction mixture was chromatographed using 10:1 hexanes/EtOAc to afford $63.3 \mathrm{mg}(77 \%)$ of the indicated compound as an oil: ${ }^{1} \mathrm{H}$ NMR $\left(400 \mathrm{MHz}, \mathrm{CDCl}_{3}\right) \delta$ 7.87-7.81 (m, 2H), 7.70-7.68 (m, 1H), 7.45-7.25 (m, 5H), $6.93(\mathrm{~s}, 1 \mathrm{H}), 5.20(\mathrm{~s}, 2 \mathrm{H})$, 4.30-4.24 (m, 4H), 1.29-1.26 (m, 6H); $\left.{ }^{13} \mathrm{C} \mathrm{NMR} \mathrm{(100} \mathrm{MHz,} \mathrm{CDCl}_{3}\right) \delta$ 167.3, 159.1, 149.2, 142.8, 140.4, 140.2, 133.0, 130.0, 128.8, 126.3, 125.3, 121.5, 115.9, 109.4, 70.6, 66.1, 62.4, 13.9; IR (neat, $\mathrm{cm}^{-1}$ ) 3445, 2983, 1732, 1531, 1248, 1046; Anal.Calcd for $\mathrm{C}_{22} \mathrm{H}_{21} \mathrm{NO}_{7}$ : C 64.23; H 5.14; N 3.40. Found: C 64.29; H 5.12; N 3.24.

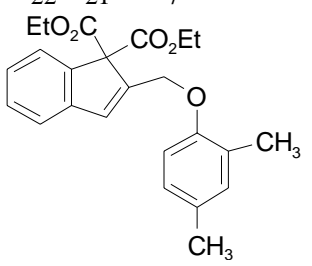

3j: The reaction mixture was chromatographed using 10:1 hexanes/EtOAc to afford $63.0 \mathrm{mg}(80 \%)$ of the indicated compound as a solid: $\mathrm{mp}: 63-65{ }^{\circ} \mathrm{C} ;{ }^{1} \mathrm{H}$ NMR (400 $\left.\mathrm{MHz}, \mathrm{CDCl}_{3}\right) \delta$ 7.67-7.65 (d, $\left.J=7.6 \mathrm{~Hz}, 1 \mathrm{H}\right), 7.33-7.21(\mathrm{~m}, 3 \mathrm{H}), 6.98-6.91(\mathrm{~m}, 3 \mathrm{H})$, 6.84-6.82 (d, $J=8.4 \mathrm{~Hz}, 1 \mathrm{H}), 5.07(\mathrm{~s}, 2 \mathrm{H}), 4.25-4.20(\mathrm{~m}, 4 \mathrm{H}), 2.28(\mathrm{~s}, 3 \mathrm{H}), 2.25(\mathrm{~s}$, $3 \mathrm{H}), 1.26-1.23(\mathrm{~m}, 6 \mathrm{H}) ;{ }^{13} \mathrm{C} \mathrm{NMR}\left(100 \mathrm{MHz}, \mathrm{CDCl}_{3}\right) \delta 167.6,154.6,143.3,142.1$, $140.5,132.0,131.5,129.6,128.7,126.9,126.4,125.9,125.1,121.3,111.3,70.6,65.5$, 62.2, 20.4, 16.2, 13.9; IR $\left(\mathrm{KBr}, \mathrm{cm}^{-1}\right)$ 3472, 2982, 1733, 1504, 1251, 1224, 1048; Anal.Calcd for $\mathrm{C}_{24} \mathrm{H}_{26} \mathrm{O}_{5}$ : C 73.08; H 6.64. Found: C 72.96; H 6.54.

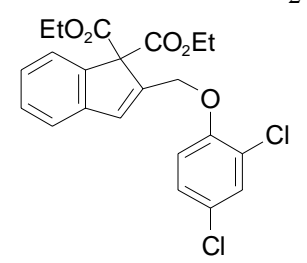

3k: The reaction mixture was chromatographed using 10:1 hexanes/EtOAc to afford $70.9 \mathrm{mg}(81 \%)$ of the indicated compound as an oil: ${ }^{1} \mathrm{H}$ NMR $\left(400 \mathrm{MHz}, \mathrm{CDCl}_{3}\right) \delta$ 7.68-7.66 (dd, $J=6.8,0.8 \mathrm{~Hz}, 1 \mathrm{H}), 7.39-7.38(\mathrm{~d}, J=2.8 \mathrm{~Hz}, 1 \mathrm{H}), 7.34-7.23(\mathrm{~m}, 3 \mathrm{H})$, 7.17-7.14 (m, 1H), 7.05-7.03 (d, $J=9.2 \mathrm{~Hz}, 1 \mathrm{H}), 6.95(\mathrm{~s}, 1 \mathrm{H}), 5.17(\mathrm{~s}, 2 \mathrm{H}), 4.27-4.21$ (m, 4H), 1.28-1.24 (m, 6H); ${ }^{13} \mathrm{C}$ NMR $\left(100 \mathrm{MHz}, \mathrm{CDCl}_{3}\right) \delta 167.4,152.9,142.9,140.3$, 140.2, 132.8, 129.9, 128.8, 127.6, 126.1, 125.8, 125.2, 123.5, 121.6, 114.5, 70.6, 66.6, 62.4, 13.9; IR (neat, $\mathrm{cm}^{-1}$ ) 3465, 2983, 1731, 1482, 1251, 1052; Anal.Calcd for $\mathrm{C}_{22} \mathrm{H}_{20} \mathrm{Cl}_{2} \mathrm{O}_{5}$ : C 60.70; $\mathrm{H} 4$ 4.63. Found: $\mathrm{C} 60.47 ; \mathrm{H} 4.54$. 


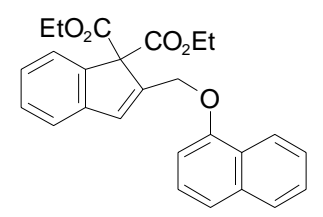

31: The reaction mixture was chromatographed using 10:1 hexanes/EtOAc to afford $37.4 \mathrm{mg}(45 \%)$ of the indicated compound as a solid: mp: 92-94 ${ }^{\circ} \mathrm{C} ;{ }^{1} \mathrm{H}$ NMR (400 $\left.\mathrm{MHz}, \mathrm{CDCl}_{3}\right) \delta 8.37-8.34(\mathrm{~m}, 1 \mathrm{H}), 7.82-7.80(\mathrm{~m}, 1 \mathrm{H}), 7.70-7.68(\mathrm{dd}, J=7.6,1.2 \mathrm{~Hz}$, $1 \mathrm{H}), 7.50-7.43(\mathrm{~m}, 3 \mathrm{H}), 7.38-7.23(\mathrm{~m}, 4 \mathrm{H}), 7.06(\mathrm{~s}, 1 \mathrm{H}), 6.98-6.96(\mathrm{~m}, 1 \mathrm{H}), 5.29(\mathrm{~s}$, 2H), 4.26-4.20 (m, 4H), 1.25-1.21 (m, 6H); $\left.{ }^{13} \mathrm{C} \mathrm{NMR} \mathrm{(100} \mathrm{MHz,} \mathrm{CDCl}_{3}\right) \delta 167.6$, 154.3, 143.3, 141.5, 140.6, 134.6, 132.8, 128.8, 127.5, 126.4, 126.1, 125.9, 125.7, $125.2,122.1,121.5,120.4,105.2,70.8,65.6,62.3,13.9 ; \mathrm{IR}\left(\mathrm{KBr}, \mathrm{cm}^{-1}\right) 3449,2982$, 1731, 1579, 1462, 1396, 1238, 1049; Anal.Calcd for $\mathrm{C}_{26} \mathrm{H}_{24} \mathrm{O}_{5}$ : C 74.98; H 5.81. Found: C 74.74; H 5.72.

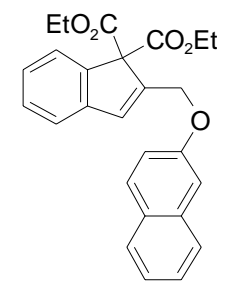

3m: The reaction mixture was chromatographed using 10:1 hexanes/EtOAc to afford $67.4 \mathrm{mg}(81 \%)$ of the indicated compound as a solid: mp: $122-124{ }^{\circ} \mathrm{C} ;{ }^{1} \mathrm{H}$ NMR $(400$ $\left.\mathrm{MHz}, \mathrm{CDCl}_{3}\right) \delta$ 7.76-7.68 (m, 4H), 7.43-7.39 (m, 1H), 7.34-7.21 (m, 6H), 7.00 (s, 1H), 5.22 (s, 2H), 4.28-4.22 (q, $J=7.2 \mathrm{~Hz}, 4 \mathrm{H}), 1.27-1.23(\mathrm{~m}, 6 \mathrm{H}) ;{ }^{13} \mathrm{C}$ NMR (100 MHz, $\left.\mathrm{CDCl}_{3}\right) \delta 167.6,156.5,143.2,141.3,140.5,134.5,132.8,129.4,129.0,128.8,127.6$, 126.8, 126.3, 126.1, 125.2, 123.6, 121.5, 118.7, 107.3, 70.7, 65.4, 62.3, 13.9; IR (KBr, $\mathrm{cm}^{-1}$ ) 3444, 2982, 1732, 1629, 1466, 1391, 1254, 1216 $\square 1049$; Anal.Calcd for $\mathrm{C}_{26} \mathrm{H}_{24} \mathrm{O}_{5}$ : C 74.98; H 5.81. Found: C 74.70; H 5.77.

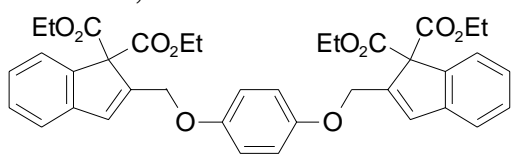

3n: The reaction mixture was chromatographed using 10:1 hexanes/EtOAc to afford $95.5 \mathrm{mg}(73 \%)$ of the indicated compound as a solid: mp: $109-111{ }^{\circ} \mathrm{C} ;{ }^{1} \mathrm{H}$ NMR $(400$ $\left.\mathrm{MHz}, \mathrm{CDCl}_{3}\right) \delta$ 7.67-7.65 (d, J=7.6 Hz, 2H), 7.33-7.22 (m, 6H), $6.94(\mathrm{~s}, 6 \mathrm{H}), 5.05(\mathrm{~s}$, $4 \mathrm{H}), 4.25-4.20$ (q, $J=7.2 \mathrm{~Hz}, 8 \mathrm{H}), 1.26-1.23(\mathrm{t}, J=7.2 \mathrm{~Hz}, 12 \mathrm{H}) ;{ }^{13} \mathrm{C}$ NMR $(100 \mathrm{MHz}$, $\left.\mathrm{CDCl}_{3}\right) \delta 167.6,153.1,143.3,141.9,140.6,132.5,128.7,126.0,125.1,121.4,115.6$, 70.6, 66.0, 62.2, 13.9; IR (KBr, $\left.\mathrm{cm}^{-1}\right)$ 3446, 2982, 1732, 1506, 1229, 1209, 1049; Anal.Calcd for $\mathrm{C}_{38} \mathrm{H}_{38} \mathrm{O}_{10}$ : C 69.71; H 5.85. Found: C 69.50; H 5.77.

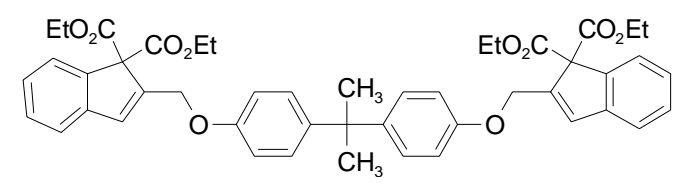

3o: The reaction mixture was chromatographed using 10:1 hexanes/EtOAc to afford $125.0 \mathrm{mg}(81 \%)$ of the indicated compound as an oil: ${ }^{1} \mathrm{H} \mathrm{NMR}\left(400 \mathrm{MHz}, \mathrm{CDCl}_{3}\right) \delta$ 7.67-7.65 (d, $J=7.2 \mathrm{~Hz}, 2 \mathrm{H}), 7.33-7.21(\mathrm{~m}, 6 \mathrm{H}), 7.15-7.13(\mathrm{dd}, J=7.2,1.6 \mathrm{~Hz}, 4 \mathrm{H})$, 
6.95-6.90 (m, 6H), $5.08(\mathrm{~s}, 4 \mathrm{H}), 4.25-4.19(\mathrm{~m}, 8 \mathrm{H}), 1.63(\mathrm{~s}, 6 \mathrm{H}), 1.26-1.22(\mathrm{~m}, 12 \mathrm{H})$;

${ }^{13} \mathrm{C}$ NMR $\left(100 \mathrm{MHz}, \mathrm{CDCl}_{3}\right) \delta 167.5,156.5,143.3,141.8,140.6,132.5,128.7,127.7$, 125.9, 125.1, 121.4, 114.1, 70.6, 65.4, 62.2, 41.6, 31.0, 13.9; IR (KBr, cm $\left.{ }^{-1}\right) 3449$, 2977, 1733, 1509, 1247, 1049; Anal.Calcd for $\mathrm{C}_{47} \mathrm{H}_{48} \mathrm{O}_{10}$ : C 73.04; H 6.26. Found: $\mathrm{C}$ 73.01; H 6.10.

\section{General Procedure for the Preparation of 2-Substituted Indenes 6.}

To a solution of 3-(2-(di(ethoxycarbonyl)methyl)phenyl)prop-2-ynyl methyl carbonate $(1 ; 69.6 \mathrm{mg}, 0.20 \mathrm{mmol})$ in DMF $(2.0 \mathrm{~mL})$ was added $\mathrm{K}_{2} \mathrm{CO}_{3}(55.2 \mathrm{mg}, 0.40 \mathrm{mmol})$, amines $(0.24 \mathrm{mmol})$, and $\mathrm{Pd}\left(\mathrm{PPh}_{3}\right)_{4}(11.5 \mathrm{mg}, 5 \mathrm{~mol} \%)$. The resulting mixture was then heated under an argon atmosphere at $80^{\circ} \mathrm{C}$. When the reaction was considered complete as determined by TLC analysis, the reaction mixture was cooled to room temperature, quenched with a saturated aqueous solution of ammonium chloride, and the mixture was extracted with EtOAc. The combined organic extracts were washed with water and saturated brine. The organic layers were dried over $\mathrm{Na}_{2} \mathrm{SO}_{4}$ and filtered. Solvents were evaporated under reduced pressure. The residue was purified by chromatography on silica gel to afford corresponding 2-substitued indenes 6.

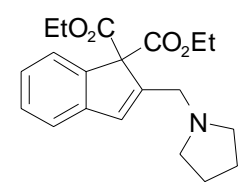

6a: The reaction mixture was chromatographed using 10:1 hexanes/EtOAc to afford $42.5 \mathrm{mg}(62 \%)$ of the indicated compound as an oil: ${ }^{1} \mathrm{H}$ NMR $\left(400 \mathrm{MHz}, \mathrm{CDCl}_{3}\right) \delta$ 7.61-7.59 (d, $J=8.0 \mathrm{~Hz}, 1 \mathrm{H}), 7.32-7.24(\mathrm{~m}, 2 \mathrm{H}), 7.21-7.17(\mathrm{~m}, 1 \mathrm{H}), 6.88(\mathrm{~s}, 1 \mathrm{H})$, 4.23-4.15 (m, 4H), $3.59(\mathrm{~s}, 2 \mathrm{H}), 2.63-2.60(\mathrm{t}, J=6.4 \mathrm{~Hz}, 4 \mathrm{H}), 1.81-1.78(\mathrm{~m}, 4 \mathrm{H})$, 1.30-1.22 (m, 6H); ${ }^{13} \mathrm{C}$ NMR (100 MHz, $\left.\mathrm{CDCl}_{3}\right) \delta 168.1,145.2,144.0,140.9,131.7$, 128.5, 125.5, 124.9, 120.9, 71.0, 61.8, 54.5, 54.4, 23.7, 13.9; IR (neat, $\mathrm{cm}^{-1}$ ) 3402, 2964, 1731, 1463, 1234, 1050; Anal.Calcd for $\mathrm{C}_{20} \mathrm{H}_{25} \mathrm{NO}_{4}$ : C 69.95; H 7.34; N 4.08. Found: C 69.85; H 7.37; N 3.98.

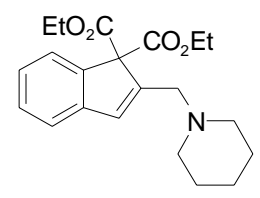

6b: The reaction mixture was chromatographed using 10:1 hexanes/EtOAc to afford $53.6 \mathrm{mg}(75 \%)$ of the indicated compound as an oil: ${ }^{1} \mathrm{H}$ NMR $\left(400 \mathrm{MHz}, \mathrm{CDCl}_{3}\right) \delta$ 7.61-7.59 (dd, $J=7.6,0.4 \mathrm{~Hz}, 1 \mathrm{H}), 7.31-7.24(\mathrm{~m}, 2 \mathrm{H}), 7.20-7.16(\mathrm{~m}, 1 \mathrm{H}), 6.86(\mathrm{~s}, 1 \mathrm{H})$, 4.23-4.16 (m, 4H), 3.39 (s, 2H), $2.46(\mathrm{~s}, 4 \mathrm{H}), 1.60-1.55(\mathrm{~m}, 4 \mathrm{H}), 1.45-1.41(\mathrm{~m}, 2 \mathrm{H})$, 1.28-1.22 (m, 6H); ${ }^{13} \mathrm{C}$ NMR (100 MHz, $\left.\mathrm{CDCl}_{3}\right) \delta 168.1,144.6,144.0,141.2,132.1$, 128.5, 125.4, 124.9, 120.9, 70.9, 61.8, 57.6, 55.0, 26.2, 24.5, 13.9; IR (neat, $\mathrm{cm}^{-1}$ ) 3431, 2934, 1731, 1465, 1236, 1049; Anal.Calcd for $\mathrm{C}_{21} \mathrm{H}_{27} \mathrm{NO}_{4}$ : C 70.56; H 7.61; N 3.92. Found: C 70.36; H 7.67; N 3.78. 


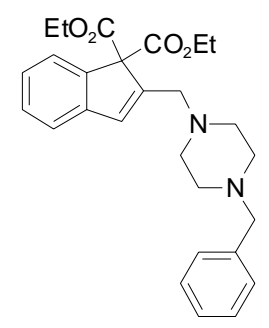

6c: The reaction mixture was chromatographed using 10:1 hexanes/EtOAc to afford $63.6 \mathrm{mg}(71 \%)$ of the indicated compound as an oil: ${ }^{1} \mathrm{H}$ NMR $\left(400 \mathrm{MHz}, \mathrm{CDCl}_{3}\right) \delta$ 7.61-7.59 (d, $J=7.6 \mathrm{~Hz}, 1 \mathrm{H}), 7.33-7.16(\mathrm{~m}, 8 \mathrm{H}), 6.84(\mathrm{~s}, 1 \mathrm{H}), 4.21-4.15(\mathrm{~m}, 4 \mathrm{H}), 3.51$ (s, 2H), 3.44 (s, 2H), 2.56-2.48 (m, 8H), 1.26-1.21(m, 6H); ${ }^{13} \mathrm{C}$ NMR (100 MHz, $\left.\mathrm{CDCl}_{3}\right) \delta 168.0,144.1,143.8,141.1,138.1,132.3,129.2,128.5,128.1,126.9,125.6$, 124.9, 120.9, 70.8, 63.0, 61.9, 56.8, 53.4, 53.2, 13.9; IR (neat, $\mathrm{cm}^{-1}$ ) 3431, 2809, 1730, 1457, 1239, 1051; Anal.Calcd for $\mathrm{C}_{27} \mathrm{H}_{32} \mathrm{~N}_{2} \mathrm{O}_{4}$ : C 72.30; H 7.19; N 6.25. Found: C 72.36; H 7.17; N 6.48 .

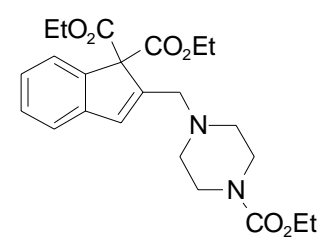

6d: The reaction mixture was chromatographed using 8:1 hexanes/EtOAc to afford $67.1 \mathrm{mg}(78 \%)$ of the indicated compound as an oil: ${ }^{1} \mathrm{H}$ NMR $\left(300 \mathrm{MHz}, \mathrm{CDCl}_{3}\right) \delta$ 7.62-7.59 (d, $J=7.5 \mathrm{~Hz}, 1 \mathrm{H}), 7.33-7.18(\mathrm{~m}, 3 \mathrm{H}), 6.87(\mathrm{~s}, 1 \mathrm{H}), 4.23-4.10(\mathrm{~m}, 6 \mathrm{H})$, $3.48-3.46(\mathrm{~m}, 6 \mathrm{H}), 2.49(\mathrm{~s}, 4 \mathrm{H}), 1.28-1.21(\mathrm{~m}, 9 \mathrm{H}) ;{ }^{13} \mathrm{C} \mathrm{NMR}\left(75 \mathrm{MHz}, \mathrm{CDCl}_{3}\right) \delta$ 167.9, 155.5, 143.6, 141.1, 132.7, 128.6, 125.8, 125.0, 121.0, 70.7, 62.0, 61.3, 56.8, 53.1, 43.8, 14.6, 13.9; IR (neat, $\mathrm{cm}^{-1}$ ) 3463, 2982, 1731, 1700, 1432, 1240, 1049; Anal.Calcd for $\mathrm{C}_{23} \mathrm{H}_{30} \mathrm{~N}_{2} \mathrm{O}_{6}$ : C 64.17; H 7.02; N 6.51. Found: C 64.07; H 7.14; N 6.43.

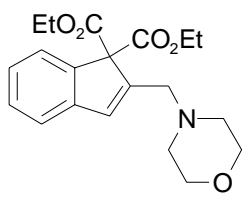

6e: The reaction mixture was chromatographed using 10:1 hexanes/EtOAc to afford $52.4 \mathrm{mg}(73 \%)$ of the indicated compound as an oil: ${ }^{1} \mathrm{H}$ NMR $\left(300 \mathrm{MHz}, \mathrm{CDCl}_{3}\right) \delta$ 7.62-7.60 (d, $J=7.5 \mathrm{~Hz}, 1 \mathrm{H}), 7.33-7.17(\mathrm{~m}, 3 \mathrm{H}), 6.87(\mathrm{~s}, 1 \mathrm{H}), 4.24-4.17$ (q, $J=7.2 \mathrm{~Hz}$, $4 \mathrm{H}), 3.72-3.69(\mathrm{t}, J=4.5 \mathrm{~Hz}, 4 \mathrm{H}), 3.45(\mathrm{~s}, 2 \mathrm{H}), 2.54-2.52(\mathrm{t}, J=4.5 \mathrm{~Hz}, 4 \mathrm{H})$, 1.27-1.23(m, 6H); ${ }^{13} \mathrm{C}$ NMR $\left(75 \mathrm{MHz}, \mathrm{CDCl}_{3}\right) \delta 167.9,143.6,143.4,141.1,132.7$, 128.5, 125.7, 124.9, 120.9, 70.7, 67.1, 61.9, 57.2, 53.9, 13.9; IR (neat, $\mathrm{cm}^{-1}$ ) 3433, 2917, 1730, 1454, 1234, 1116, 1050; Anal.Calcd for $\mathrm{C}_{20} \mathrm{H}_{25} \mathrm{NO}_{5}$ : C 66.83; H 7.01; N 3.90. Found: C 66.57; H 7.13; N 3.87.

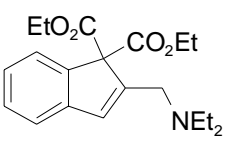

6f: The reaction mixture was chromatographed using 10:1 hexanes/EtOAc to afford $35.9 \mathrm{mg}(52 \%)$ of the indicated compound as an oil: ${ }^{1} \mathrm{H} \mathrm{NMR}\left(300 \mathrm{MHz}, \mathrm{CDCl}_{3}\right) \delta$ 7.60-7.57 (m, 1H), 7.29-7.15 (m, 3H), $6.90(\mathrm{~s}, 1 \mathrm{H}), 4.23-4.15(\mathrm{~m}, 4 \mathrm{H}), 3.49(\mathrm{~s}, 2 \mathrm{H})$, 
2.62-2.55 (m, 4H), 1.27-1.21(m, 6H), 1.06-1.01(m, 6H); $\left.{ }^{13} \mathrm{C} \mathrm{NMR} \mathrm{(75} \mathrm{MHz,} \mathrm{CDCl}_{3}\right) \delta$ 168.2, 146.2, 144.0, 141.1, 131.6, 128.5, 125.3, 124.8, 120.8, 70.7, 61.8, 52.3, 47.2, 13.9. 11.9; IR (neat, $\mathrm{cm}^{-1}$ ) 3424, 2972, 1731, 1464, 1234, 1050; Anal.Calcd for $\mathrm{C}_{20} \mathrm{H}_{27} \mathrm{NO}_{4}$ : C 69.54; H 7.88; N 4.05. Found: C 69.47; H 7.83; N 3.95.

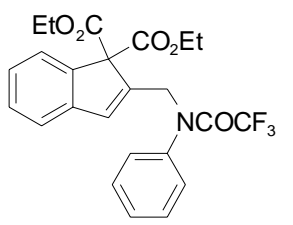

6g: The reaction mixture was chromatographed using 10:1 hexanes/EtOAc to afford $57.2 \mathrm{mg}(62 \%)$ of the indicated compound as a solid: $\mathrm{mp}: 71-73{ }^{\circ} \mathrm{C} ;{ }^{1} \mathrm{H}$ NMR (400 $\left.\mathrm{MHz}, \mathrm{CDCl}_{3}\right) \delta$ 7.63-7.61 (d, $\left.J=7.6 \mathrm{~Hz}, 1 \mathrm{H}\right), 7.39-7.37(\mathrm{~m}, 3 \mathrm{H}), 7.35-7.22(\mathrm{~m}, 5 \mathrm{H})$, $6.80(\mathrm{~s}, 1 \mathrm{H}), 4.92(\mathrm{~s}, 2 \mathrm{H}), 4.10-4.00(\mathrm{~m}, 4 \mathrm{H}), 1.16-1.12(\mathrm{t}, J=7.2 \mathrm{~Hz}, 6 \mathrm{H}) ;{ }^{13} \mathrm{C} \mathrm{NMR}$ $\left(75 \mathrm{MHz}, \mathrm{CDCl}_{3}\right) \delta 167.2,157.1\left(\mathrm{q}, J_{\mathrm{C}-\mathrm{F}}{ }^{2}=33.5 \mathrm{~Hz}\right), 142.8,140.4,139.6,133.1,129.2$, $129.0,128.8,128.1,126.2,125.4,125.2,121.4,116.4\left(\mathrm{q}, J^{1}{ }_{\mathrm{C}-\mathrm{F}}=286.5 \mathrm{~Hz}\right), 71.3,62.2$, 50.8, 13.7; IR (neat, $\mathrm{cm}^{-1}$ ) 3378, 2984, 1732, 1699, 1495, 1208, 1049; Anal.Calcd for $\mathrm{C}_{24} \mathrm{H}_{22} \mathrm{~F}_{3} \mathrm{NO}_{5}$ : C 62.47; H 4.81; N 3.04. Found: C 62.57; H 4.73; N 3.11. 


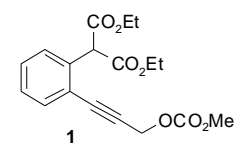

$000 \% 0-$

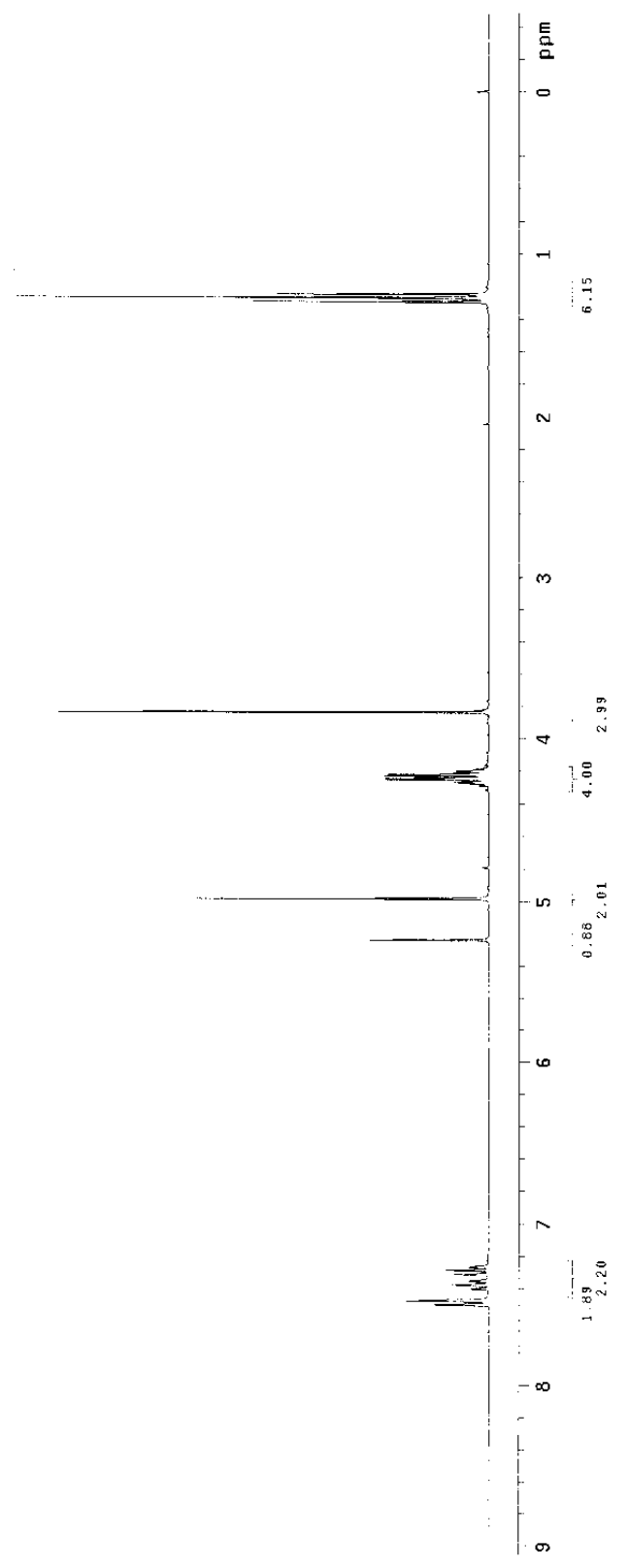

$6 \varepsilon 8^{\circ} \varepsilon-$

$861 \cdot 6$
$\angle 61 \cdot$

$\angle 6 T \cdot 0-$
$502 \cdot 0$
$022 \cdot 0-$

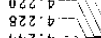

$142 \cdot t-$
$252 \cdot t$

2520

$\angle 92 \cdot 6$

$522 \cdot 0-$
$\angle 82$

$986^{\circ} \mathrm{b}$

obe $\cdot \mathrm{s}^{---}$

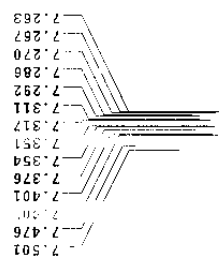




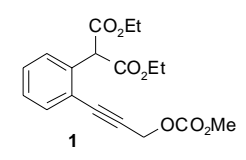

Eบ $6 \cdot \varepsilon 1 \cdots$
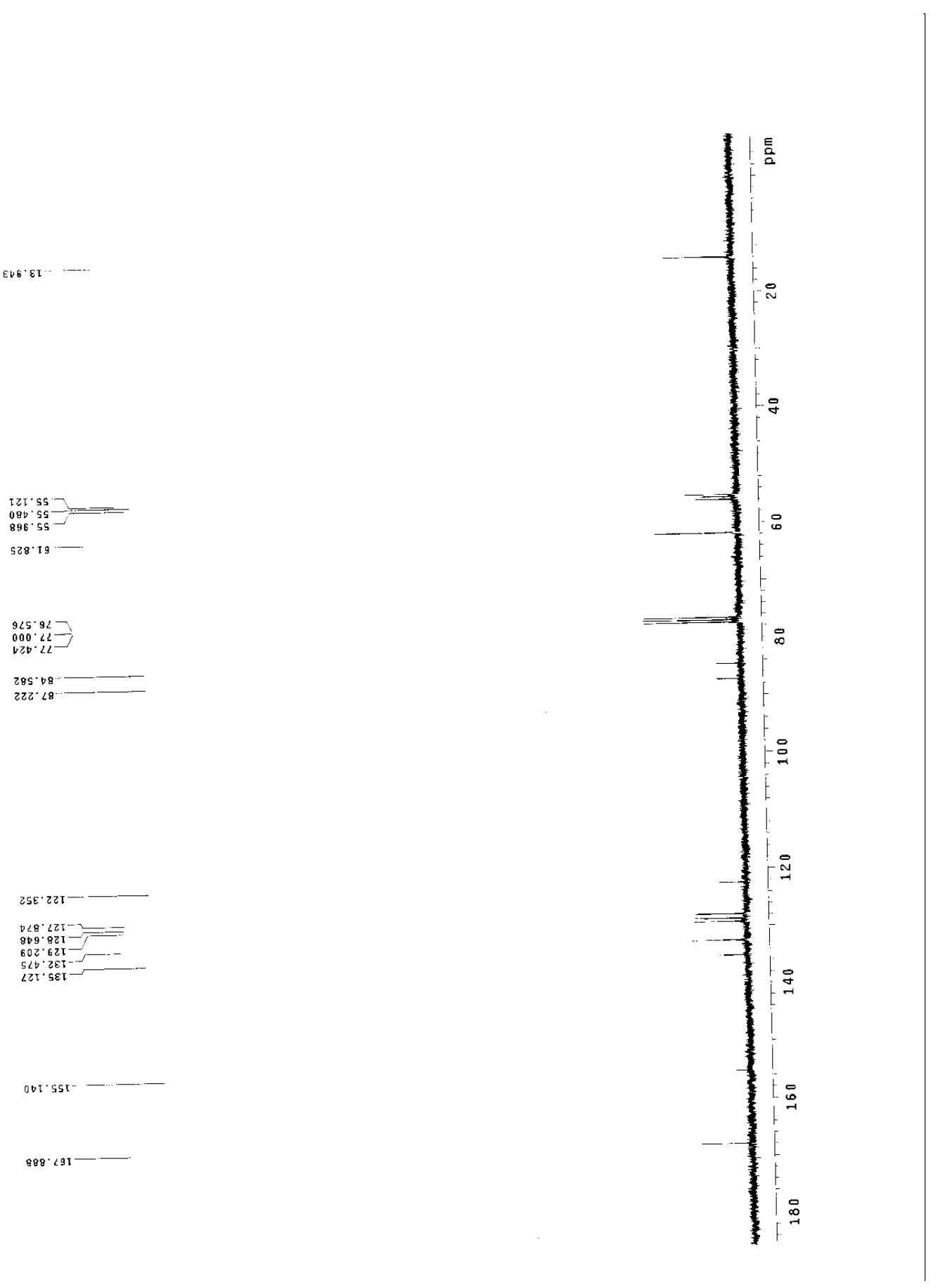

602.625
$5 \angle t-285$

ot.sst.-...-

$899 \cdot 291-$ 

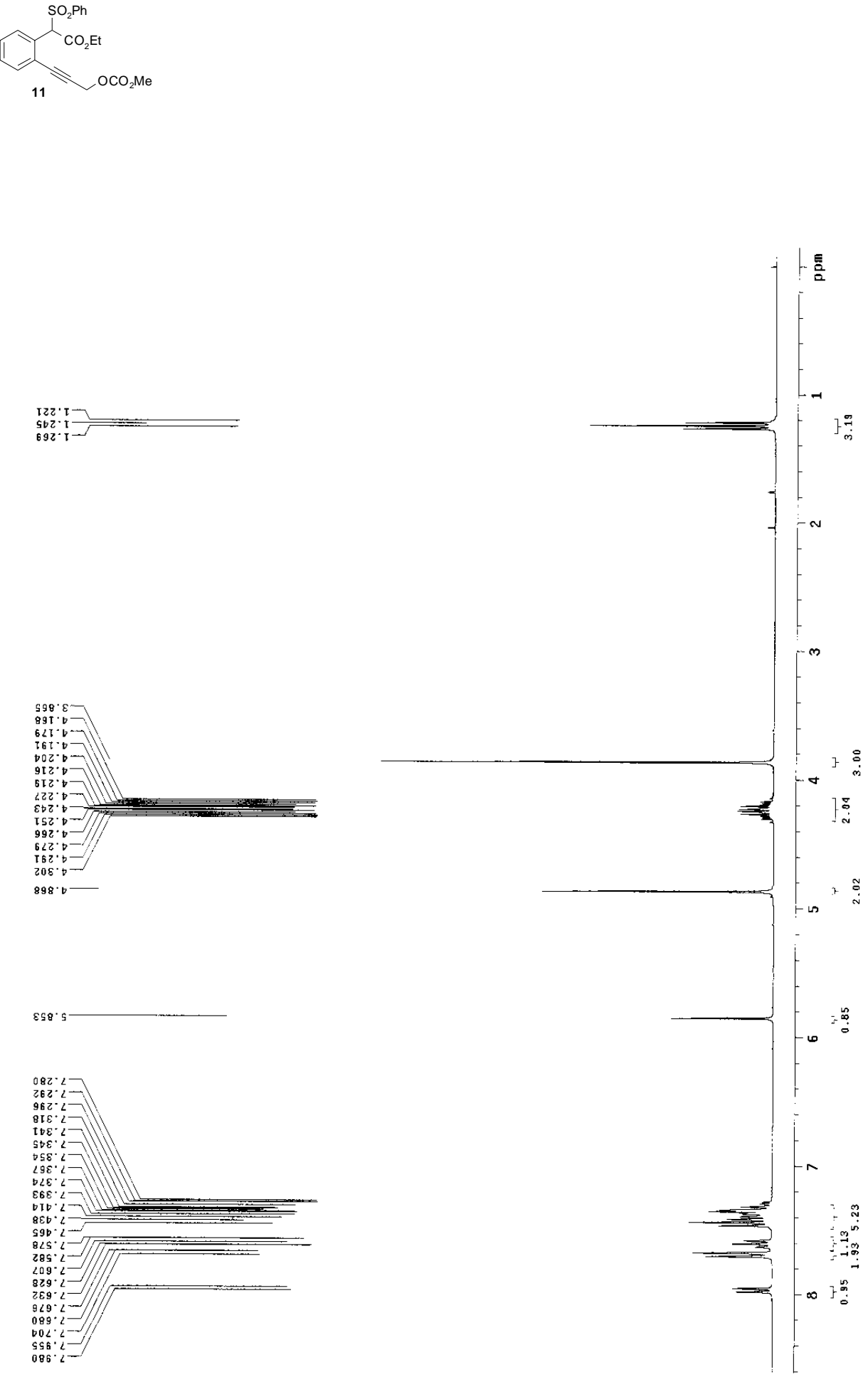

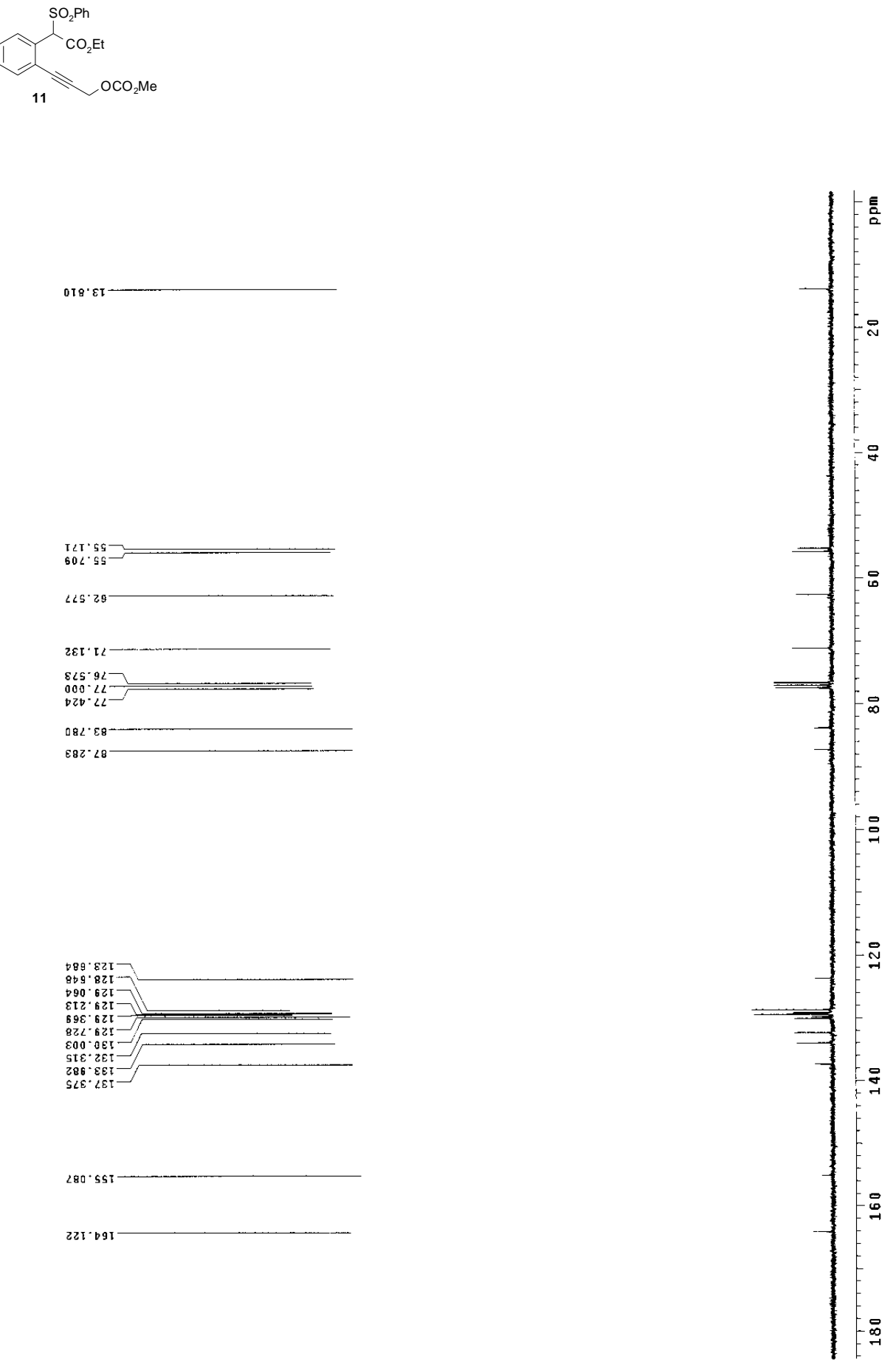

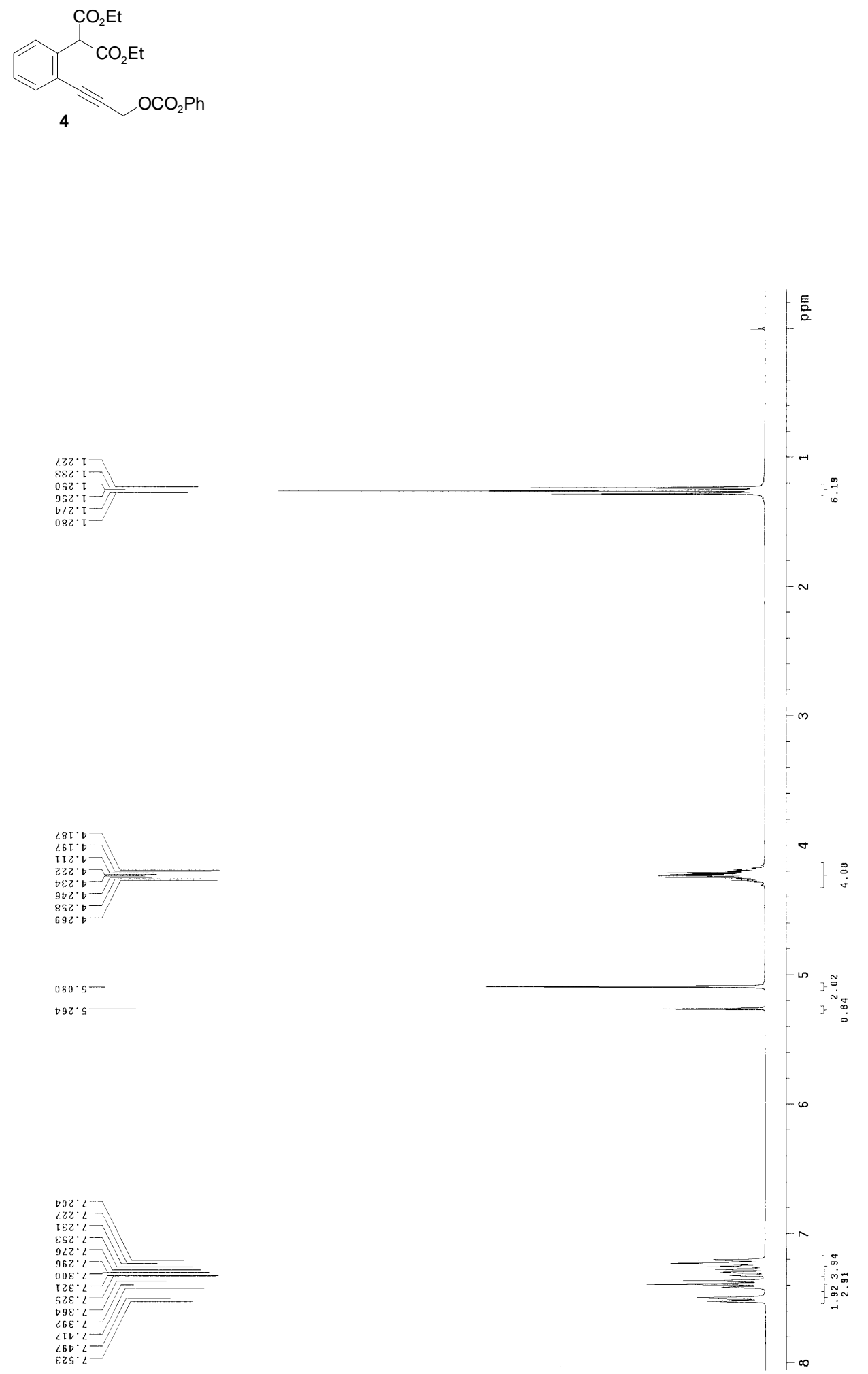

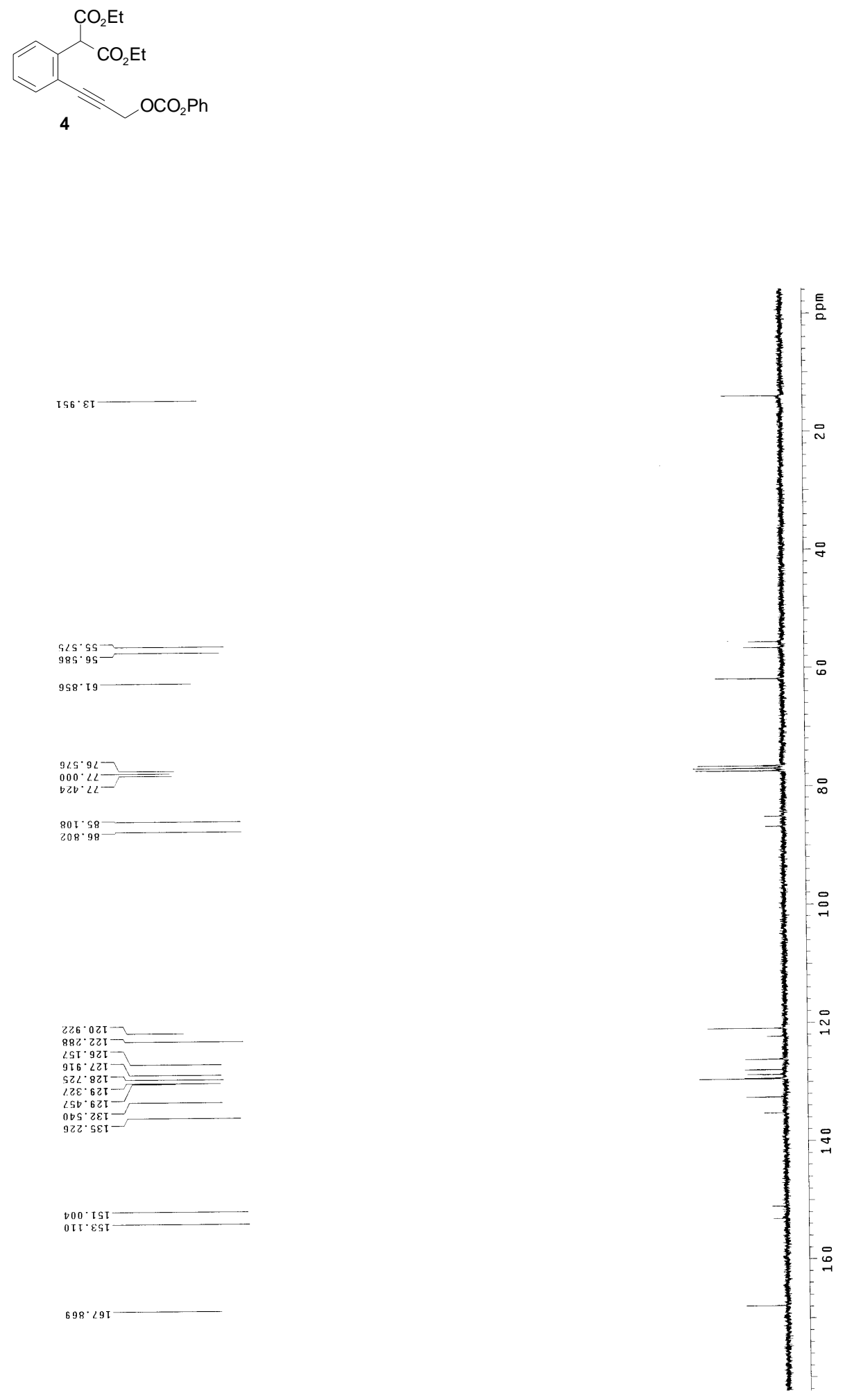


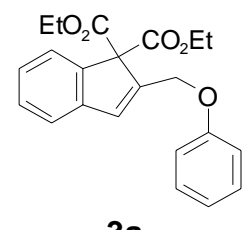

3a
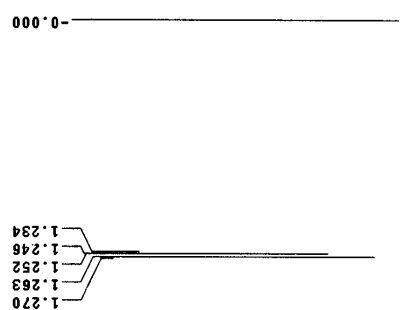

$\cos \cdot b$

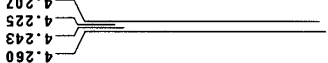

$20 \mathrm{I} \cdot \mathrm{s}$

ob6: $9-$

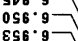

856
$8<6$
260.97

$500: 2$
$020: 2$
122.2

$\left.\begin{array}{l}620 \cdot 2 \\ t z 2 \cdot 2 \\ 622 \cdot 2 \\ 682 \cdot 2 \\ 252 \cdot 2\end{array}\right]$

$6 \varepsilon z \cdot<7$
$2 b 2 \cdot 27$
$8 b 2 \cdot 2]$

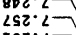

ז92. 27

$<92 \cdot 27$

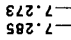

$\mathrm{MIM}_{\mathrm{M}}$

는

$208: 2-$

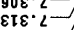

$8 \mathrm{~T} \varepsilon \varepsilon \cdot 2=$

$98 \varepsilon \cdot 2=$

$8 \varepsilon \varepsilon^{\circ}$
$b S g^{\circ}$

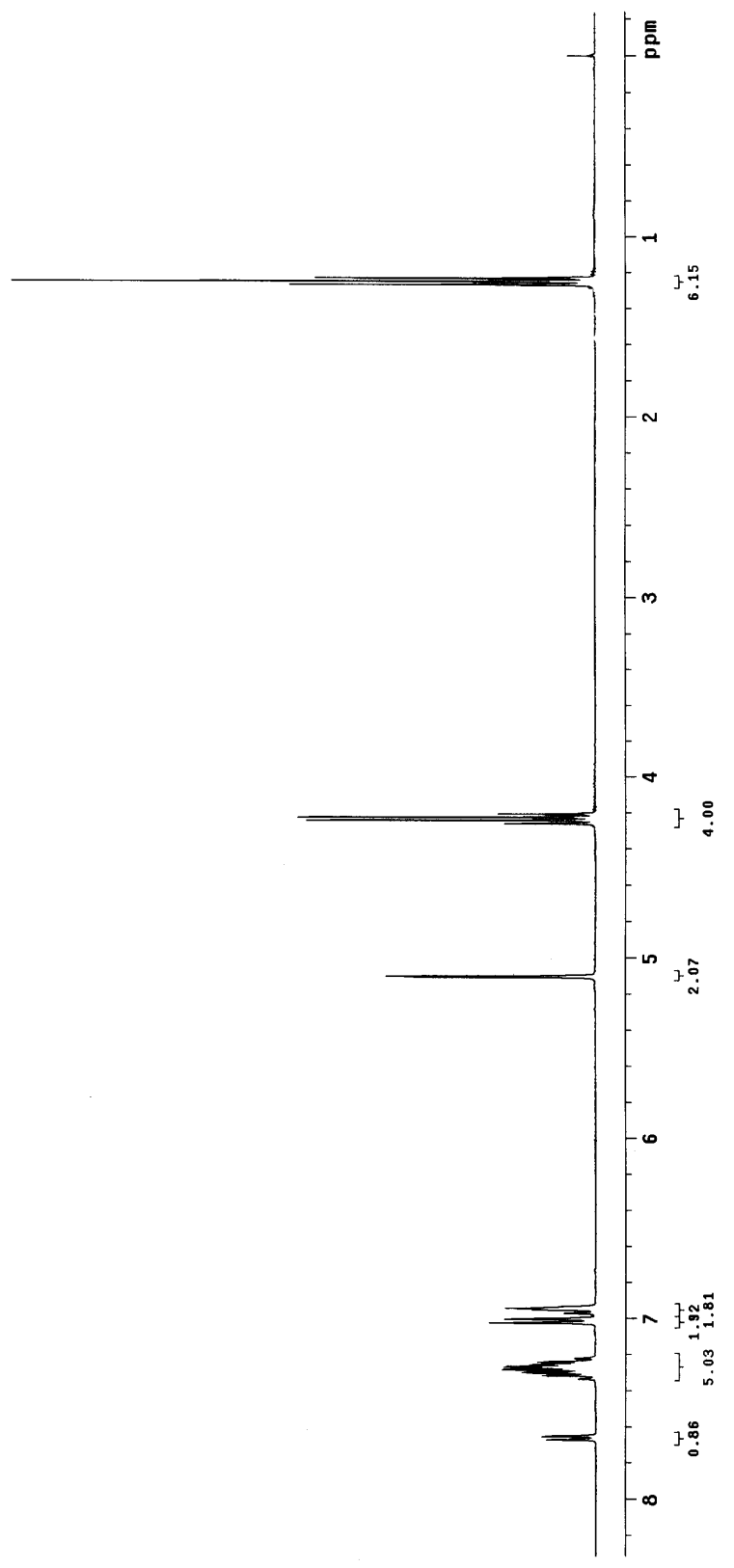




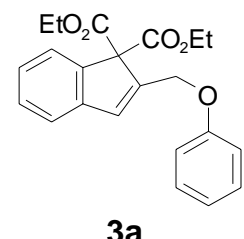

$3 a$

$926 \cdot \varepsilon \tau$

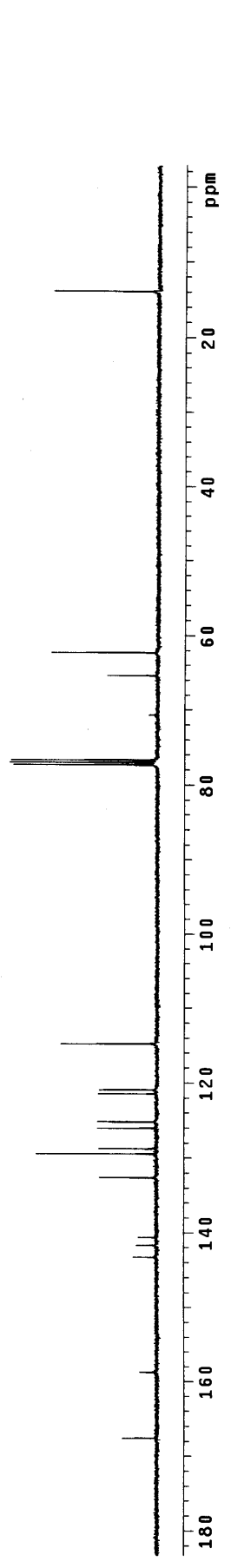

$682 \cdot 29-\square$

906.59

$889 \cdot 0<$

689.927
$000 \cdot 2 L$

$02 \varepsilon \cdot 2<$

$982 \cdot 6 \pi t$

I06 * 021

$08 \mathrm{~T} \cdot \mathrm{szt}$

$620 \cdot 921$

$0 S L \cdot 8 Z I$
$6 E D \cdot 6 Z I$
$\angle T 9 \cdot 2 \varepsilon I$

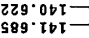

$8 \angle 2 \cdot \varepsilon b t$

$D O L \cdot 8 S T$

$86 S^{\circ} \angle 9 \tau$ 

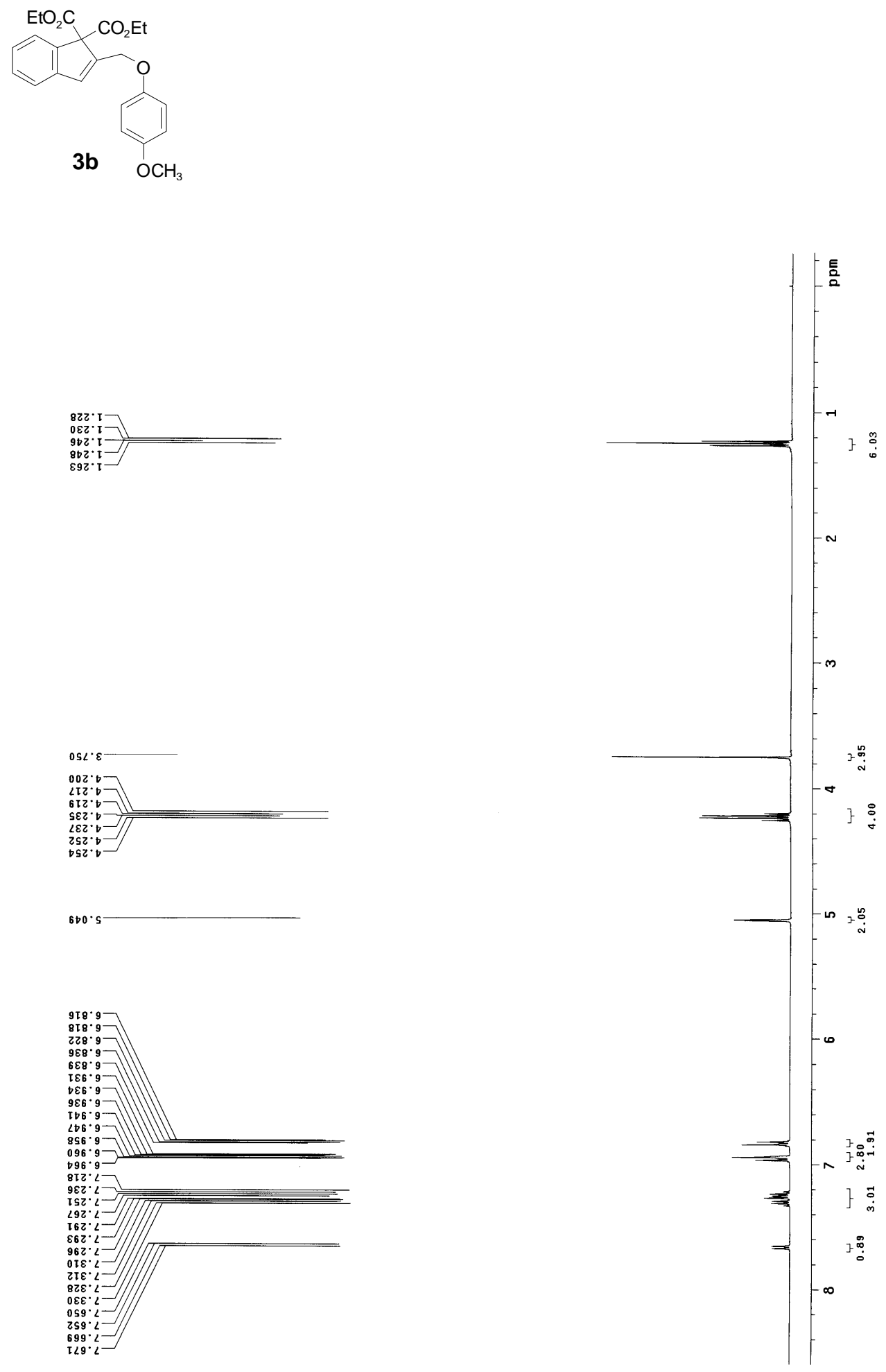


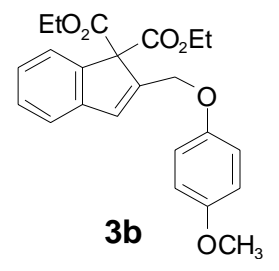

$888 \cdot \varepsilon \tau$

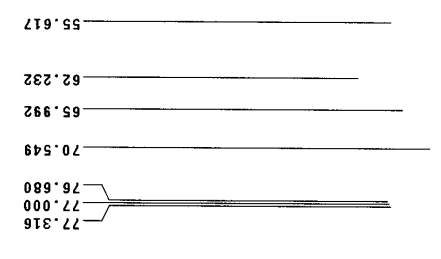

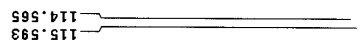

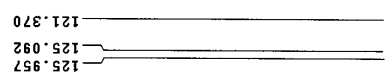

$260.521=$
$256.521=$
$002.821=$

$660 \cdot 2 \varepsilon \tau$

ז $8 \mathcal{S}^{\circ} 0 \mathrm{bt}$

$0 b \tau \cdot \varepsilon+t$

$\angle 08 \cdot 2 S T-$
$Z 88 \cdot \varepsilon S T-$

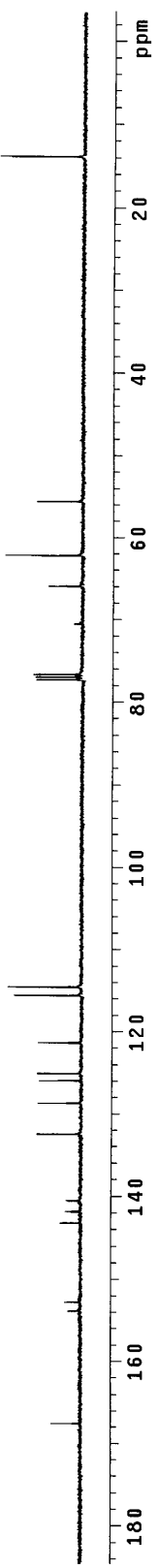



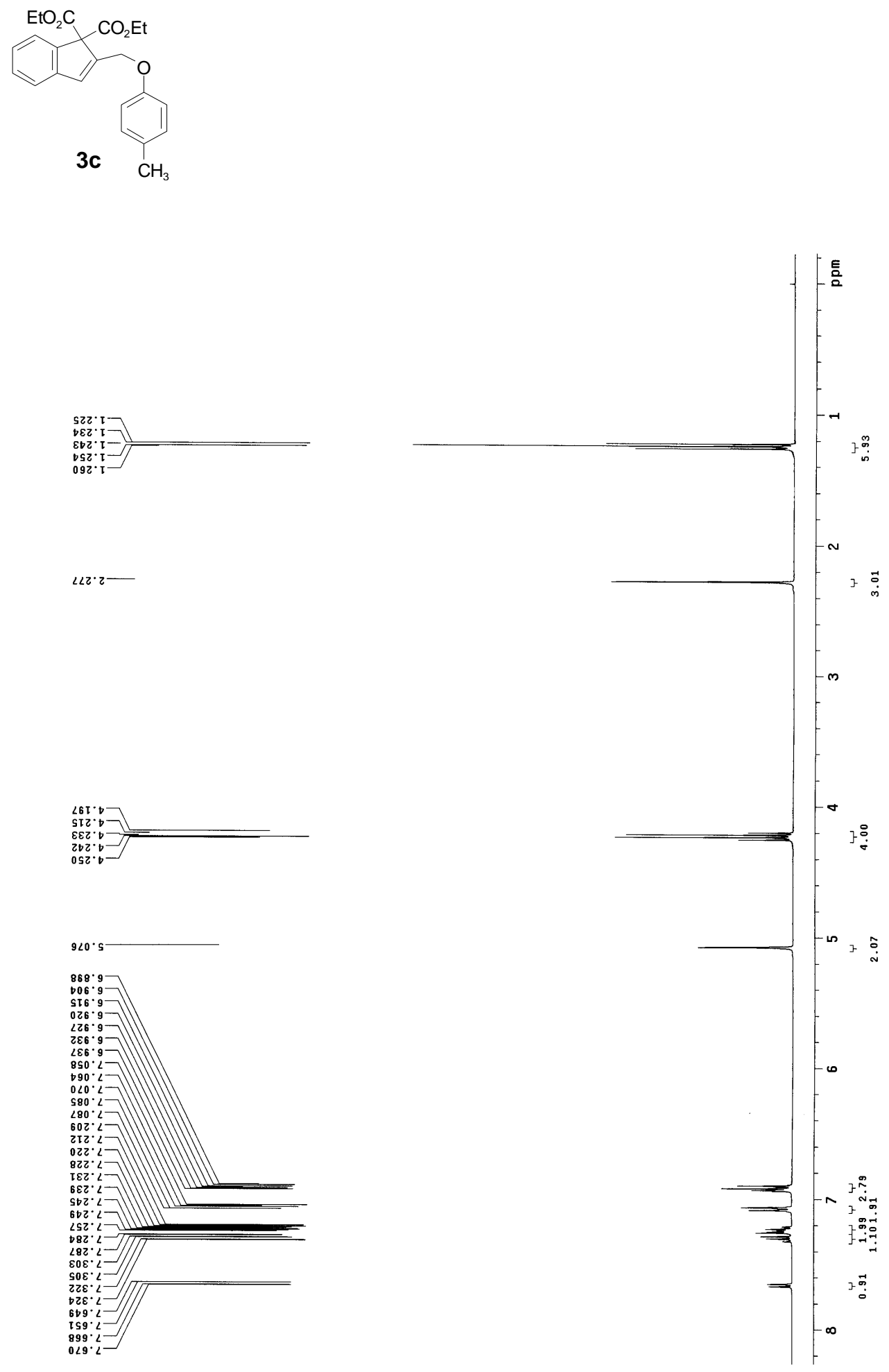

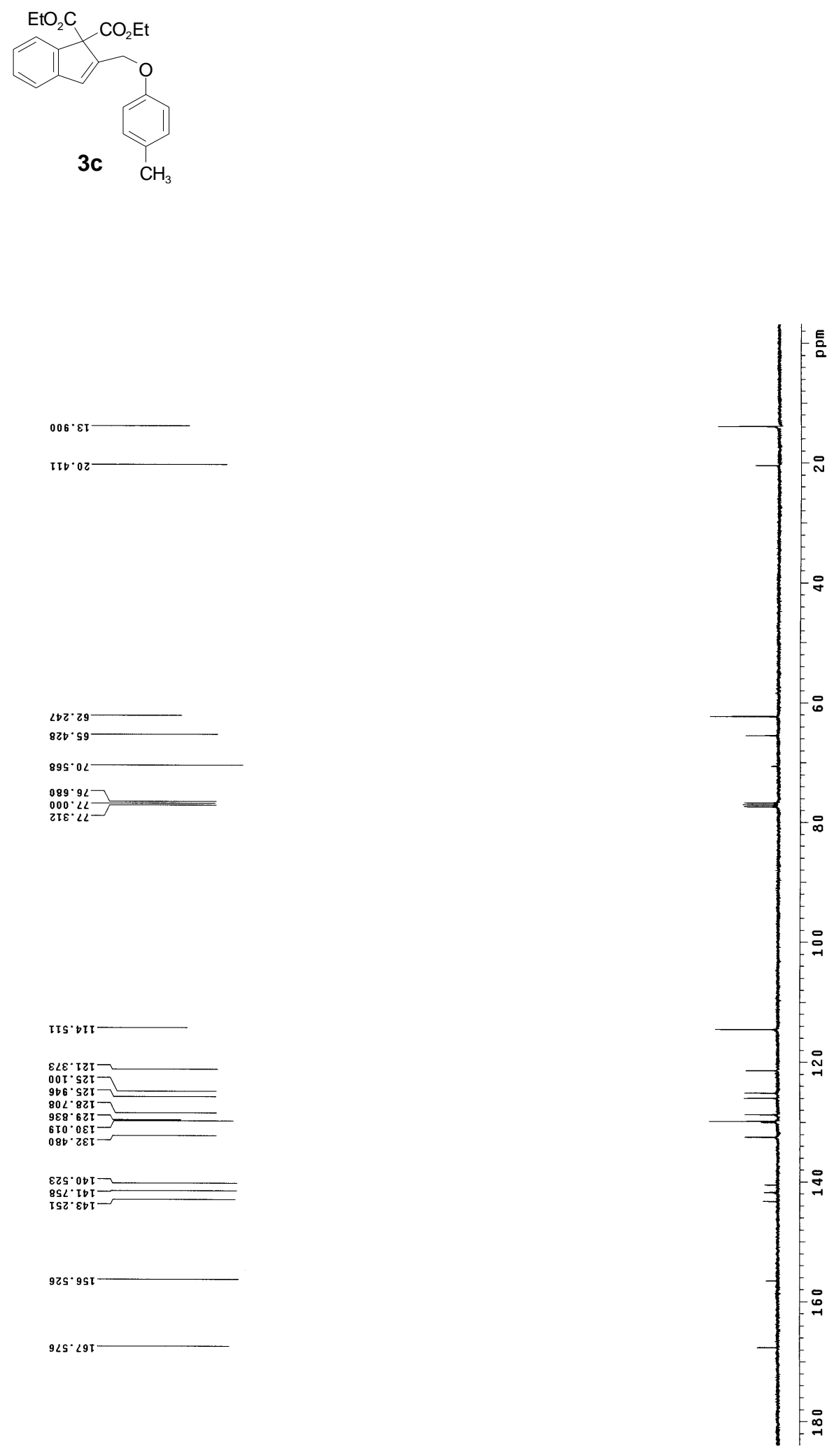

$9 \angle S \cdot \angle 9 T$ 

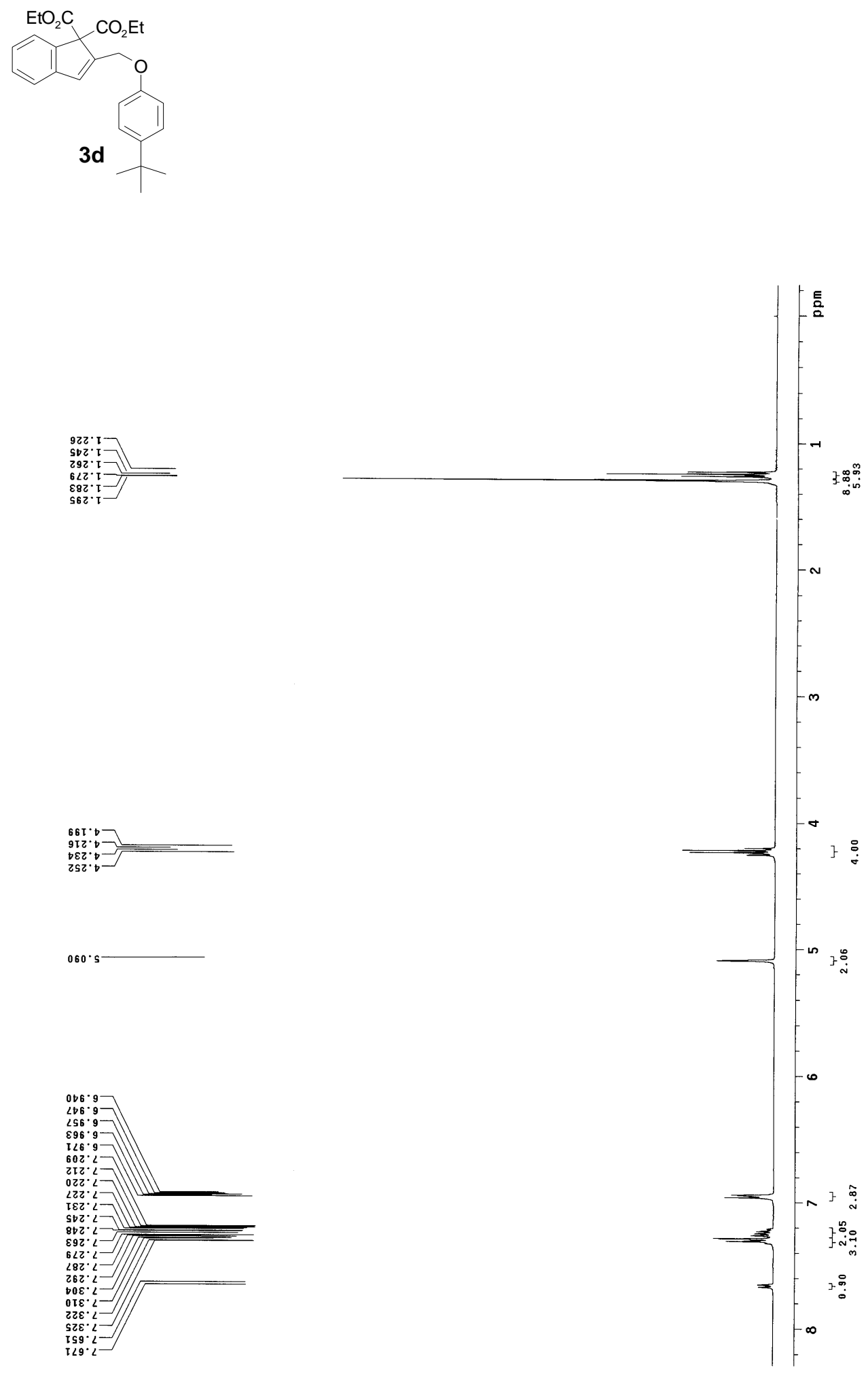


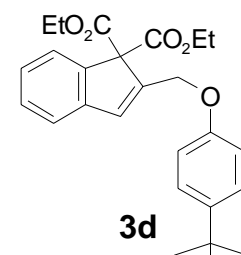

$006^{\circ} \varepsilon \mathrm{I}$

$\$ 80^{\circ} \cdot \tau \varepsilon$
$\angle \tau 0 \cdot เ \varepsilon$

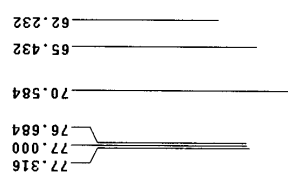

$000^{\circ} 22$

$\varepsilon S T \cdot b I$

$0<\varepsilon \cdot \tau Z \tau$

$\angle 0 T \cdot \mathrm{sZT} \longrightarrow$

686.521
$6 \mathrm{SI} \cdot 9 \mathrm{TI}$

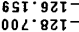

S95: $2 \varepsilon \mathfrak{-}$

$\varepsilon \angle S^{\circ} 0 b 17$

$682 \cdot 8 b 1$

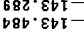

IED.9Sโ

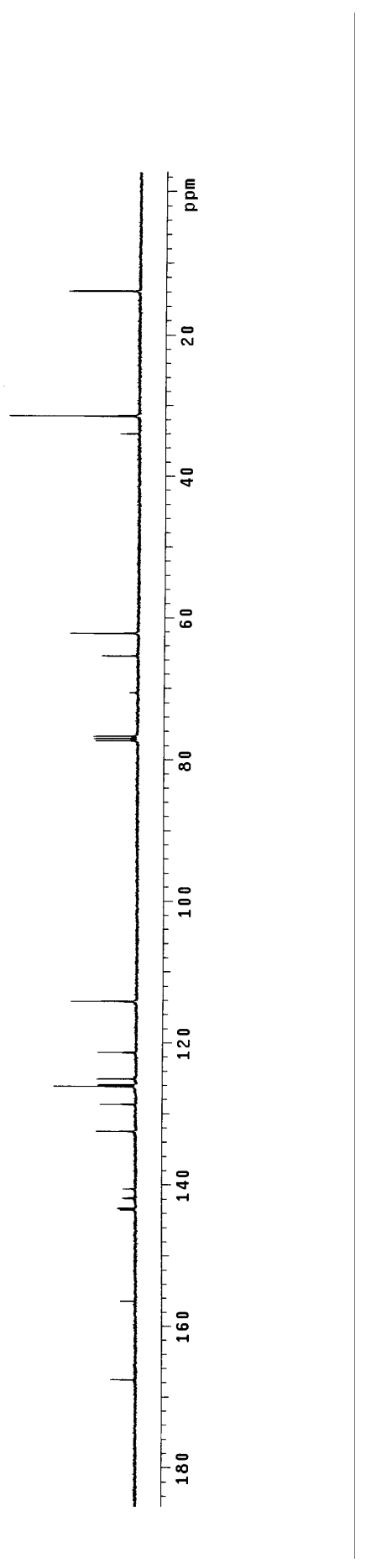

$6 \angle S \cdot \angle 9 \tau$ 

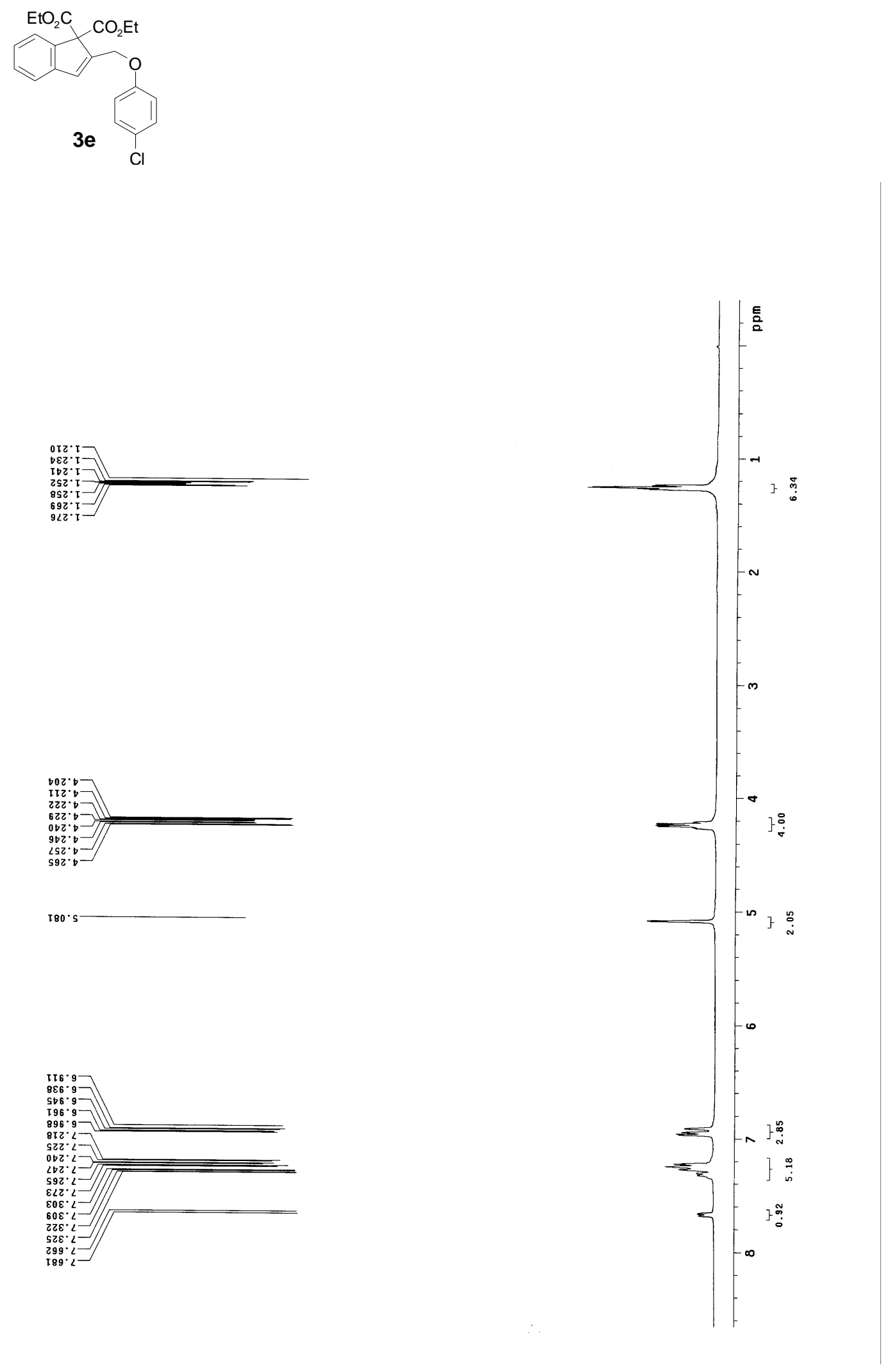


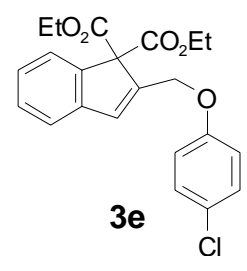

$206 \cdot \varepsilon \mathfrak{-}$

$80 \varepsilon^{\circ} 29$

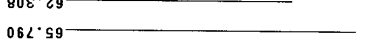

$8 \mathrm{~T} 9 \cdot 0<-$

689.92

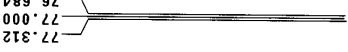

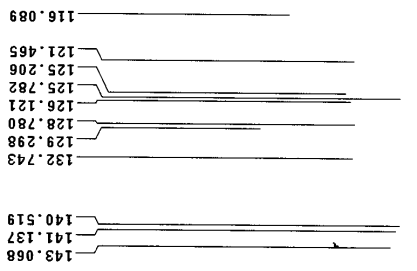

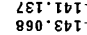

$\varepsilon 0 \varepsilon \cdot \angle S I-$

$9 \angle 0 \cdot \angle 9 \mathrm{I}$

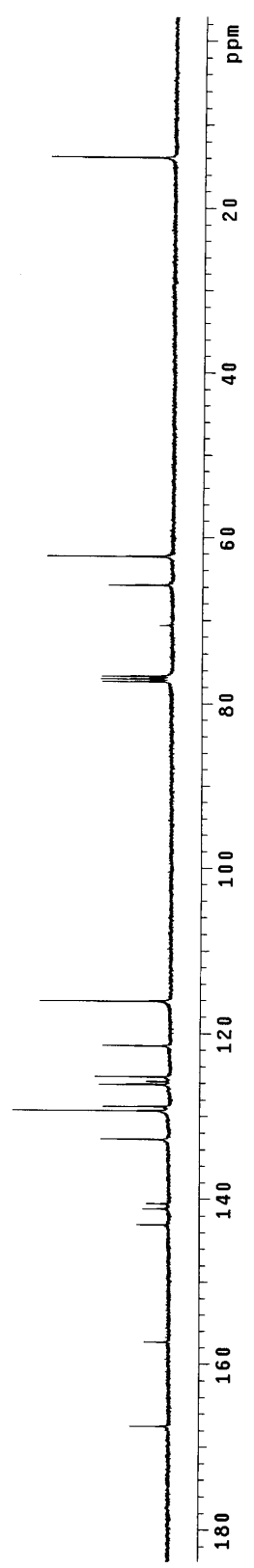



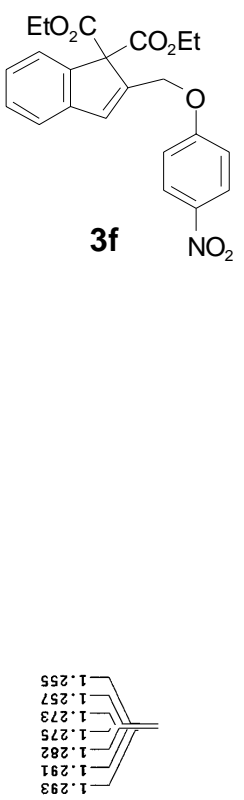

$\left.\begin{array}{l}62 z \cdot b \\ b t z \cdot t \\ 9 b 2 \cdot 0 \\ 20 \\ 0\end{array}\right]$

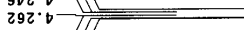

$292^{\circ} b$
$592^{\circ}$

$887^{\circ}$

$972 \cdot 5$
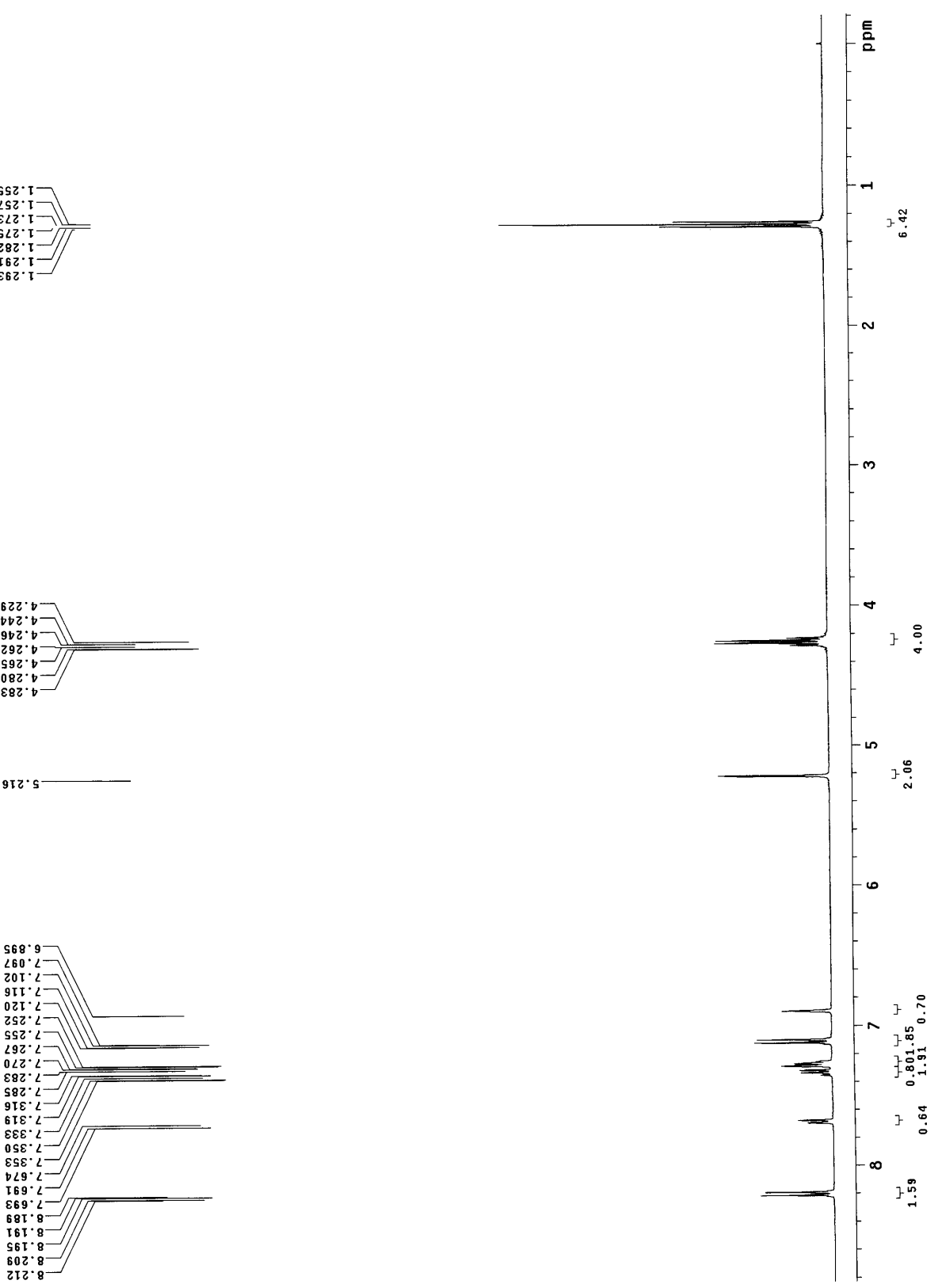


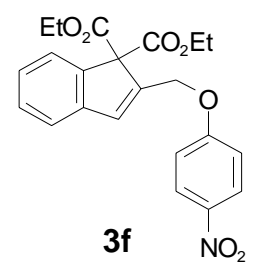

$968 \cdot \varepsilon I$
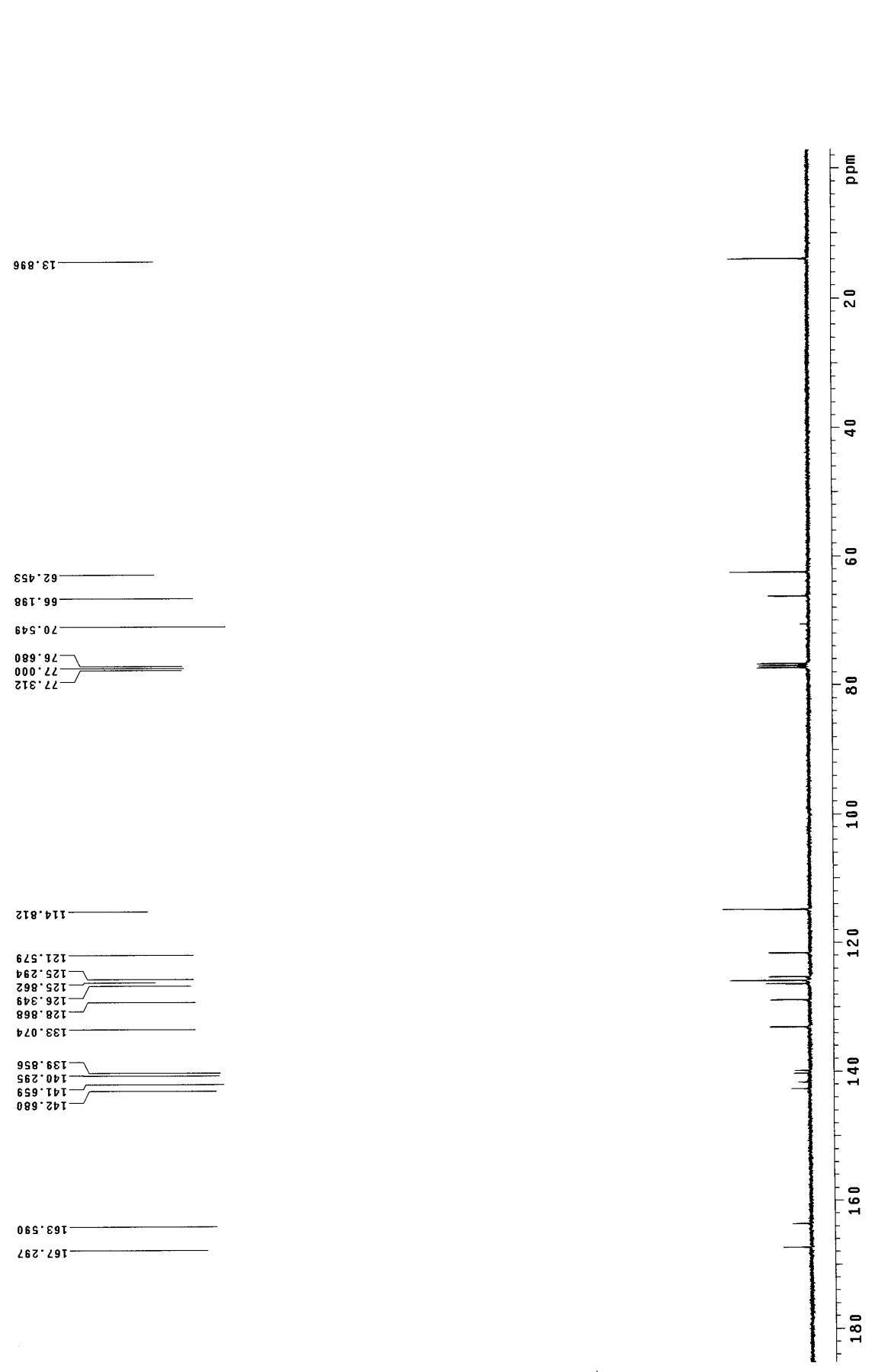

$089.92 \square \square$

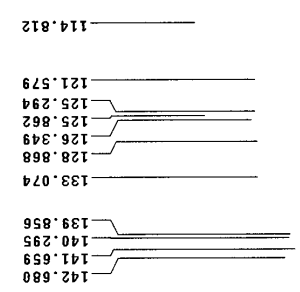

S62.00I

$659^{\circ} \mathrm{TbI}-$
$089^{\circ} \mathrm{ZDI}$

$06 \mathrm{~s} \cdot \varepsilon 9 \tau$ 

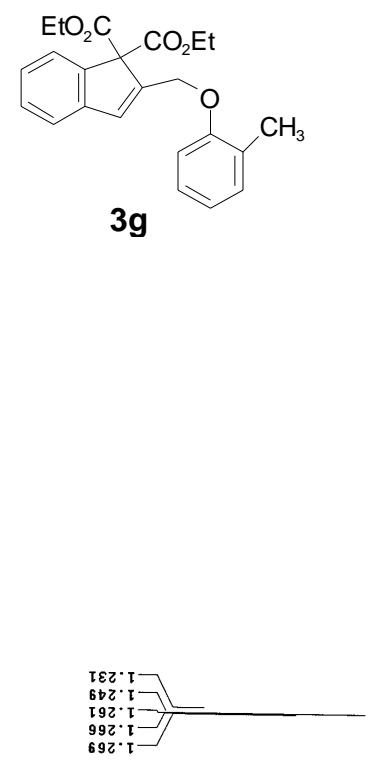

งโร.
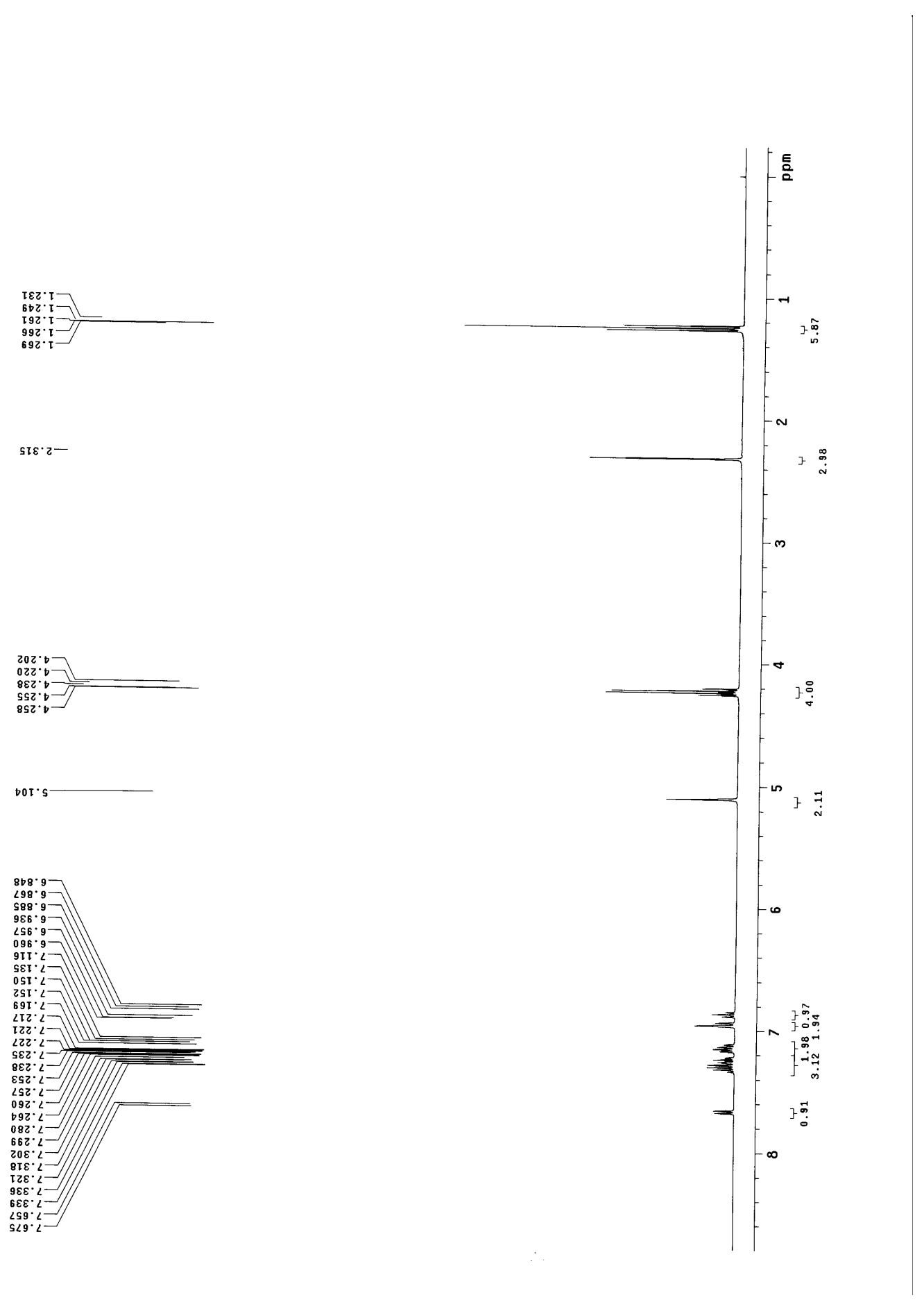

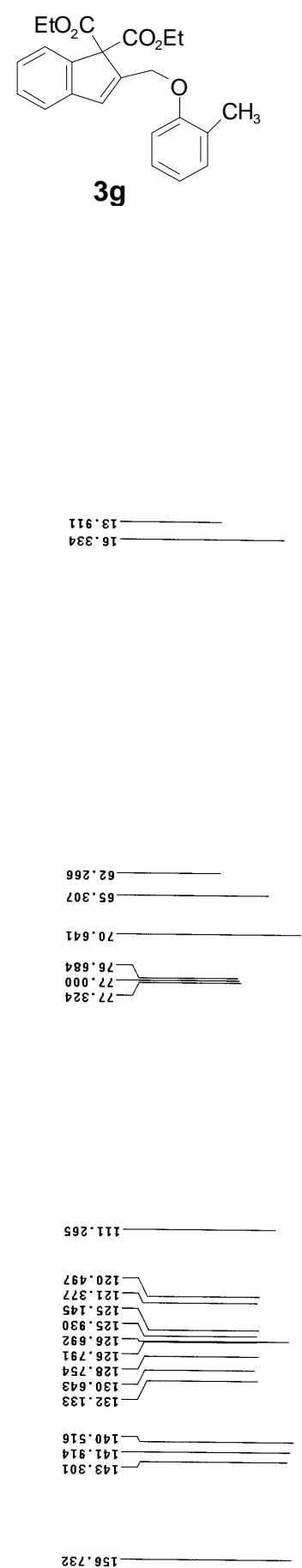

$2 \angle S \cdot \angle 9 t$

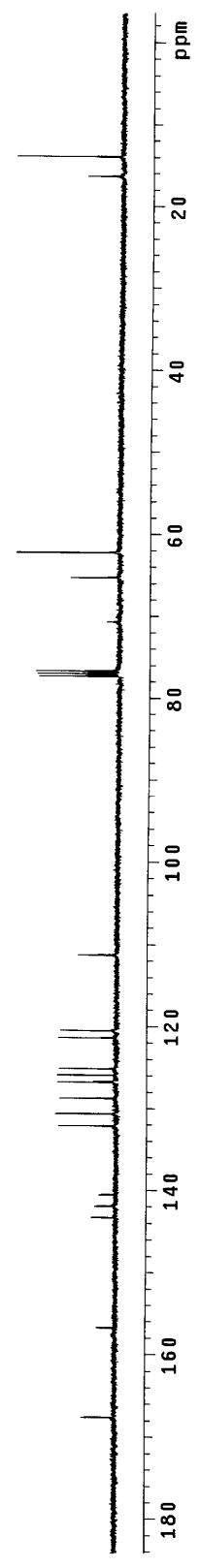




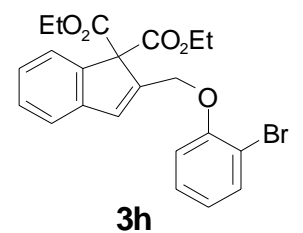

$000 \cdot 0$
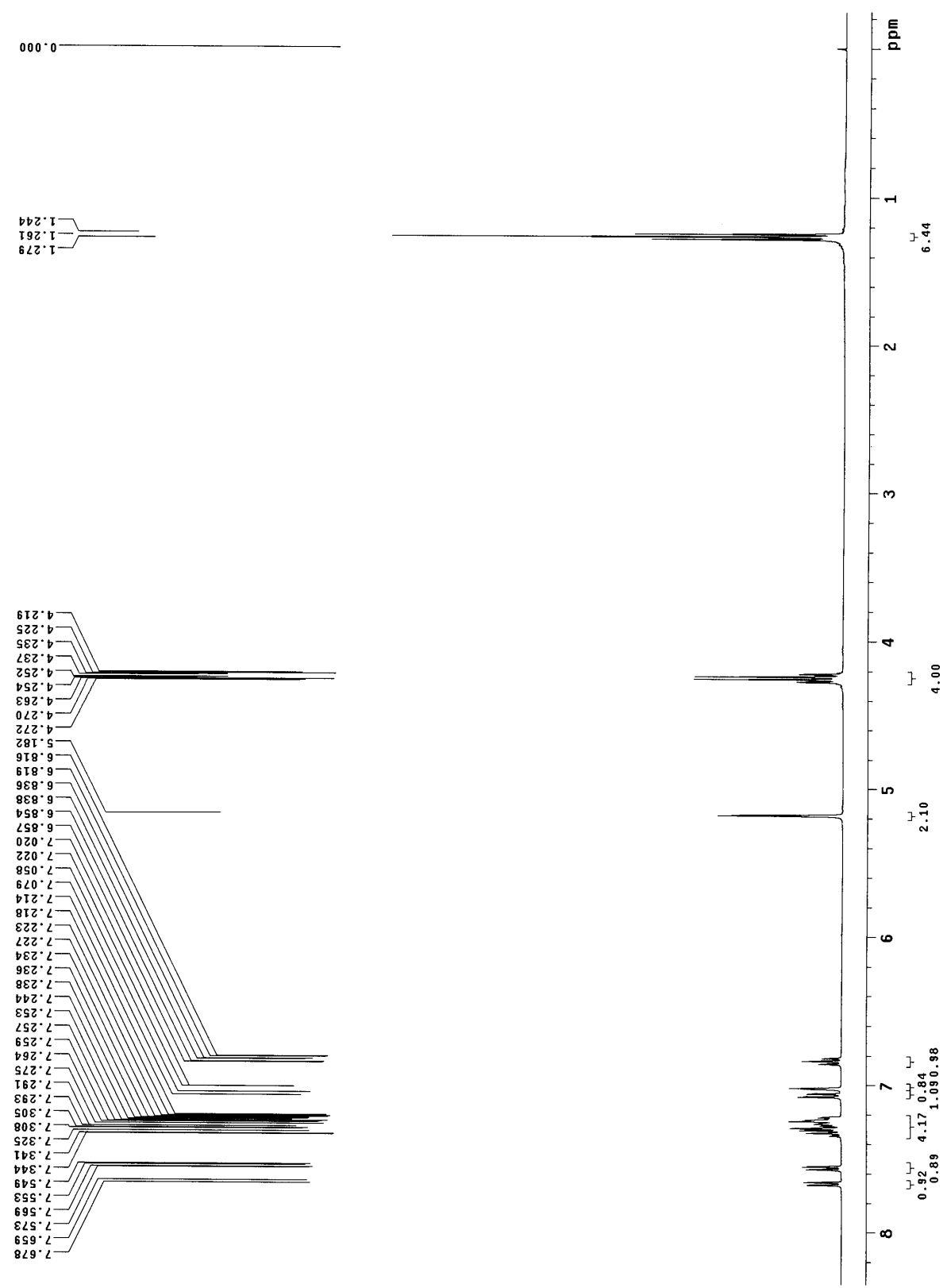


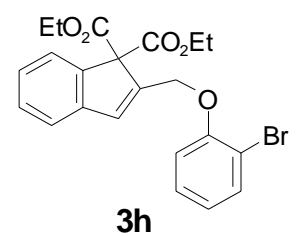

$676 \cdot \varepsilon 1$
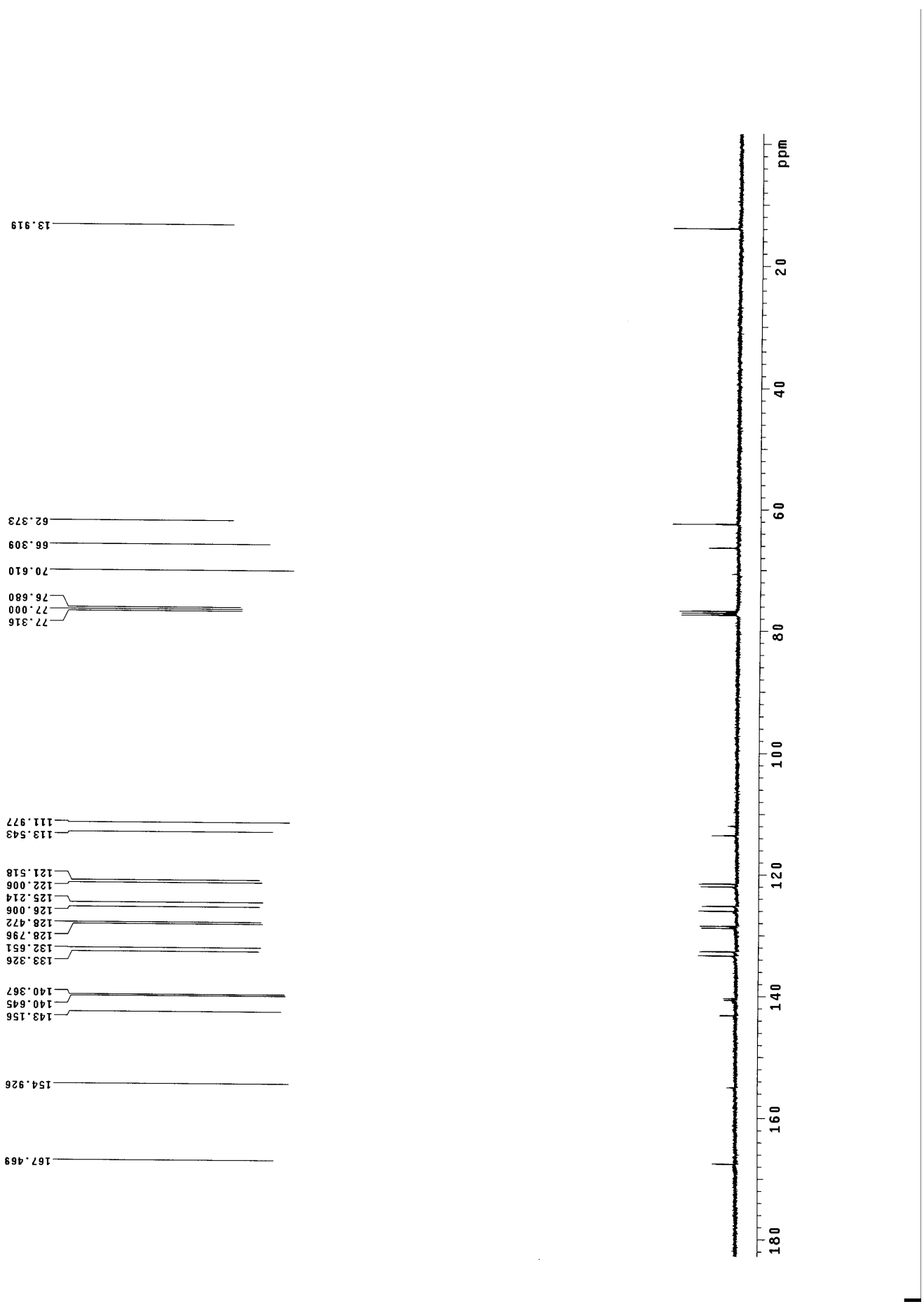

$695 \cdot \angle 9 I$ 

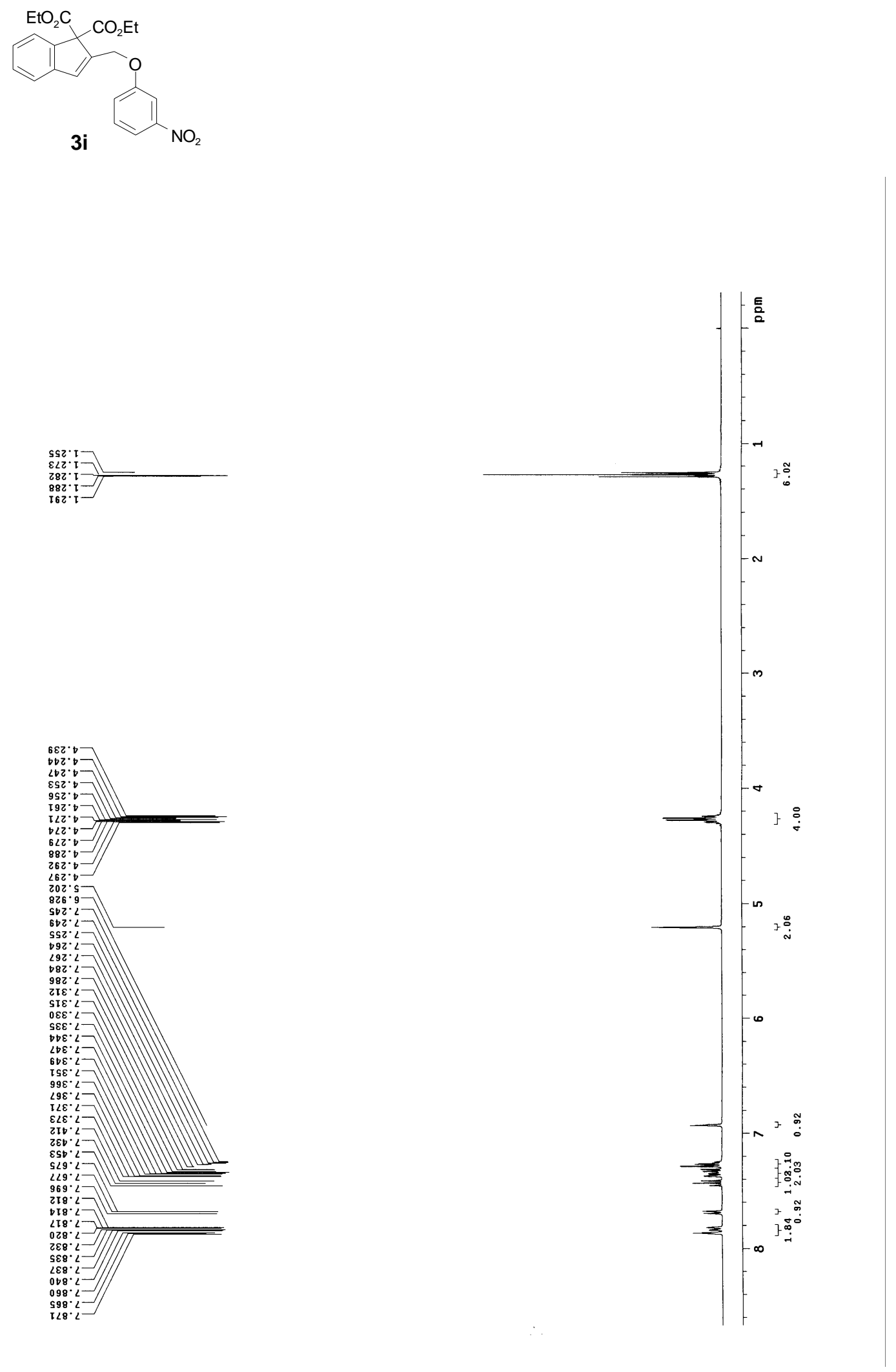


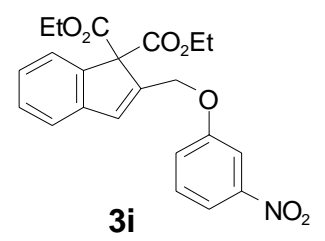

$\angle 06 \cdot \varepsilon I$

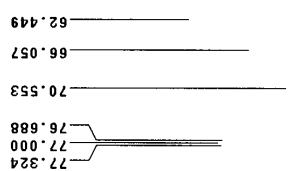

$S \angle \varepsilon^{\circ} 60 \tau$

0I6. SII -

SDS. IZI

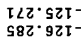

818.821

696.62

เ 9 เ 0 0อเ

$6 \angle L \cdot 2 b t$

$222 \cdot 6 \operatorname{ta}$

$6 \angle 0.65 T$

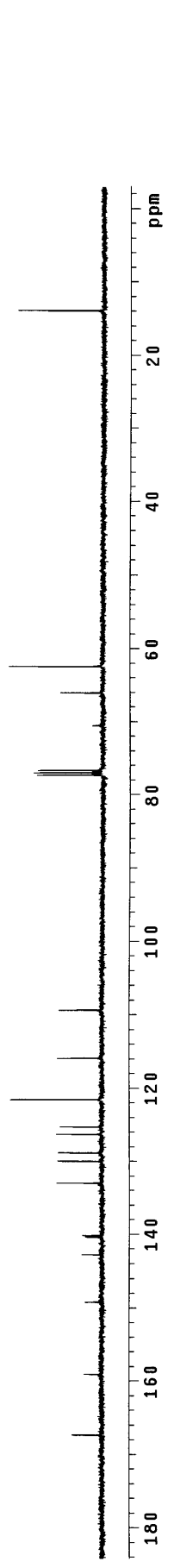

$\varsigma \varepsilon E^{\cdot} \angle 9 \tau$ 

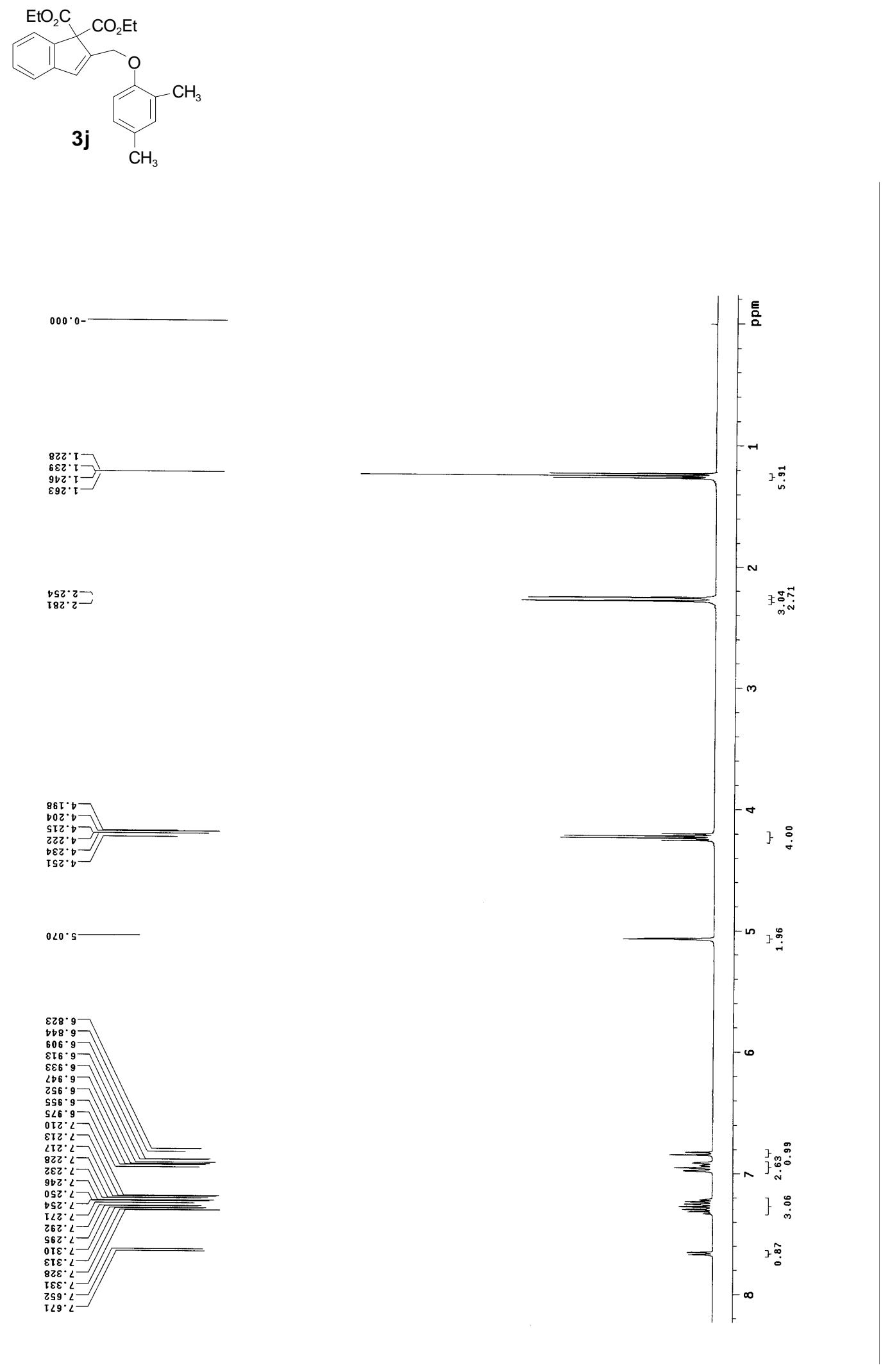

$0<0 \cdot s$

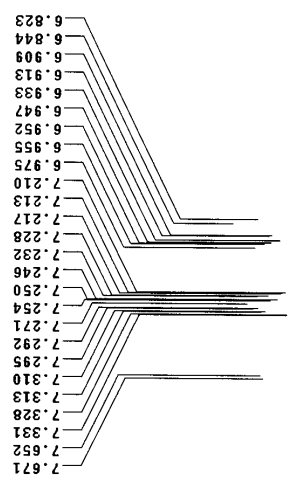



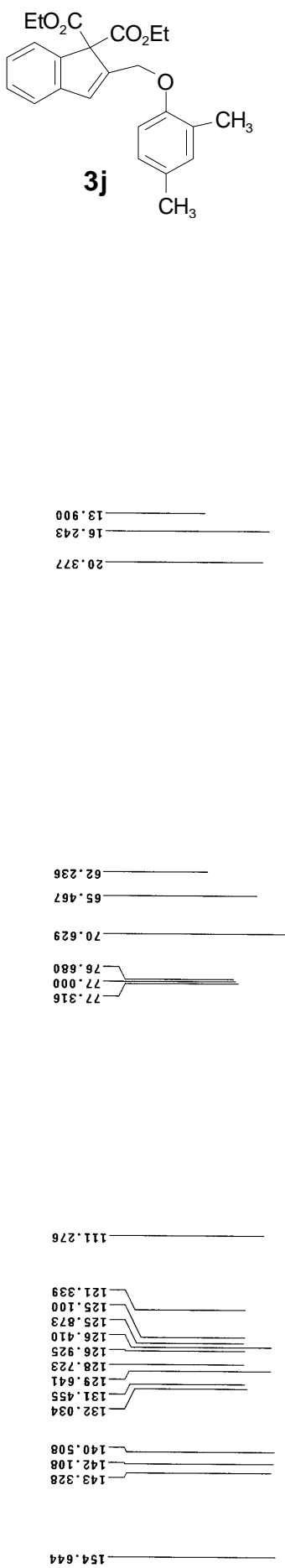

IGS' $29 \tau$

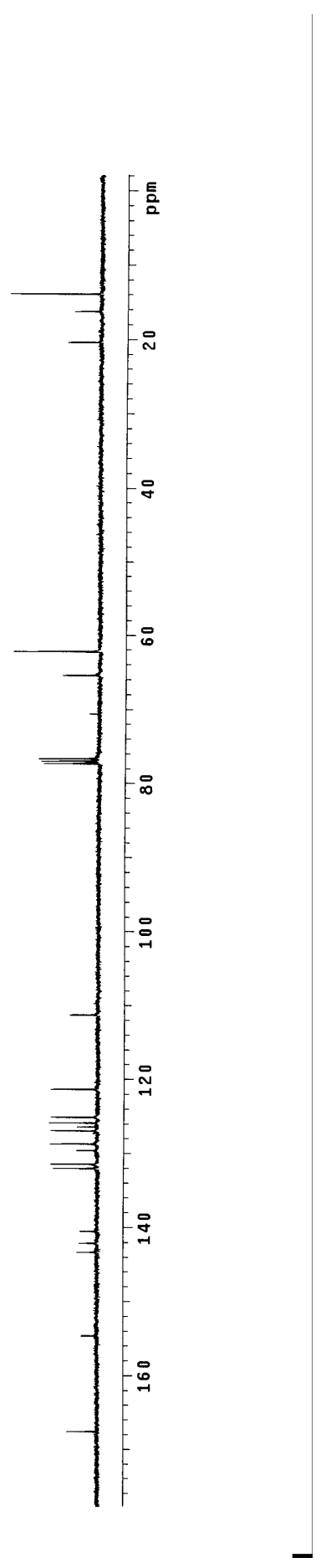



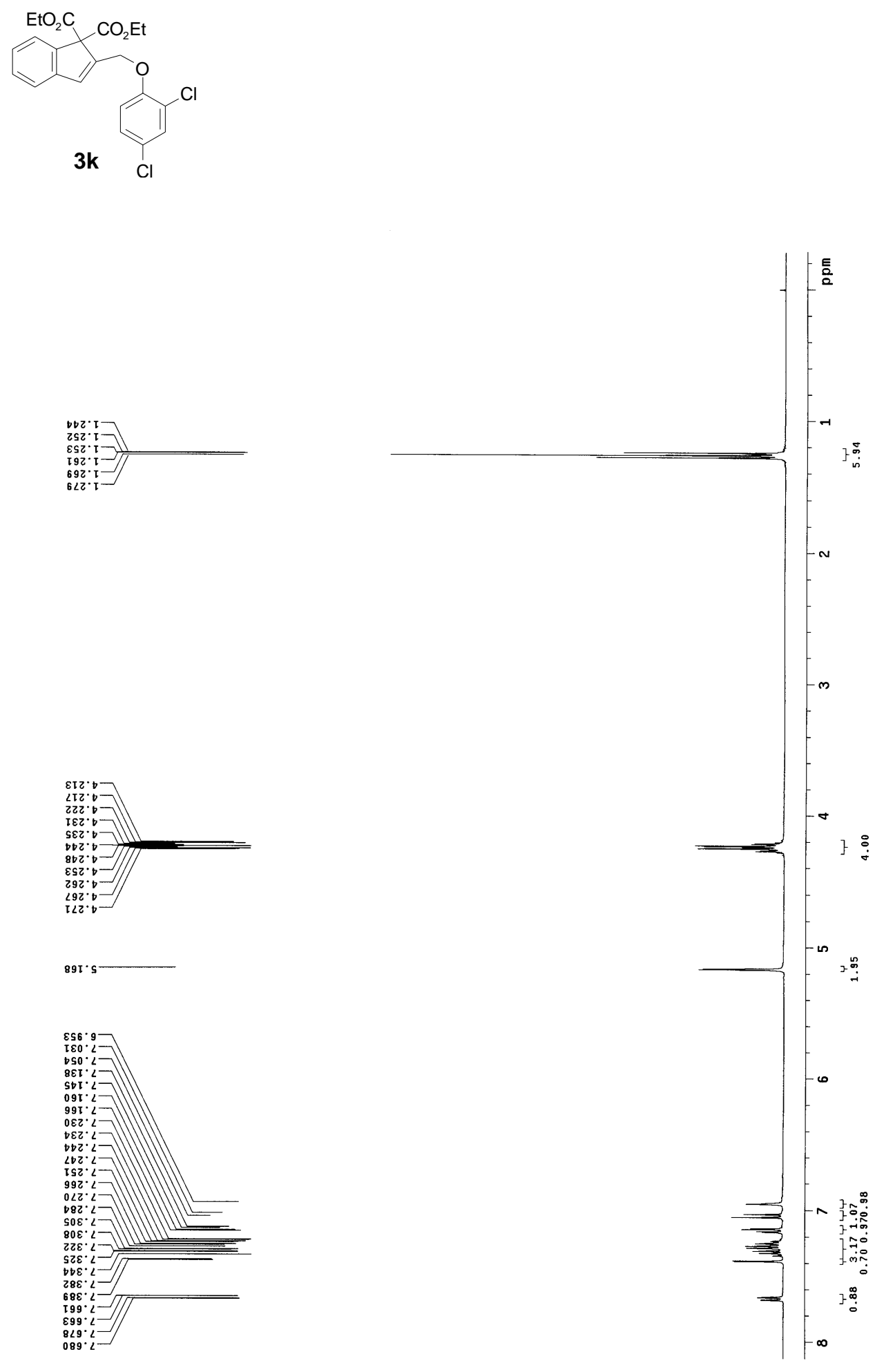

$891 \cdot s$

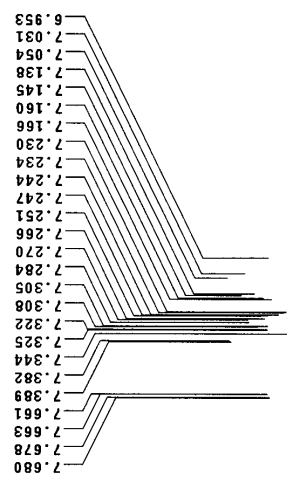



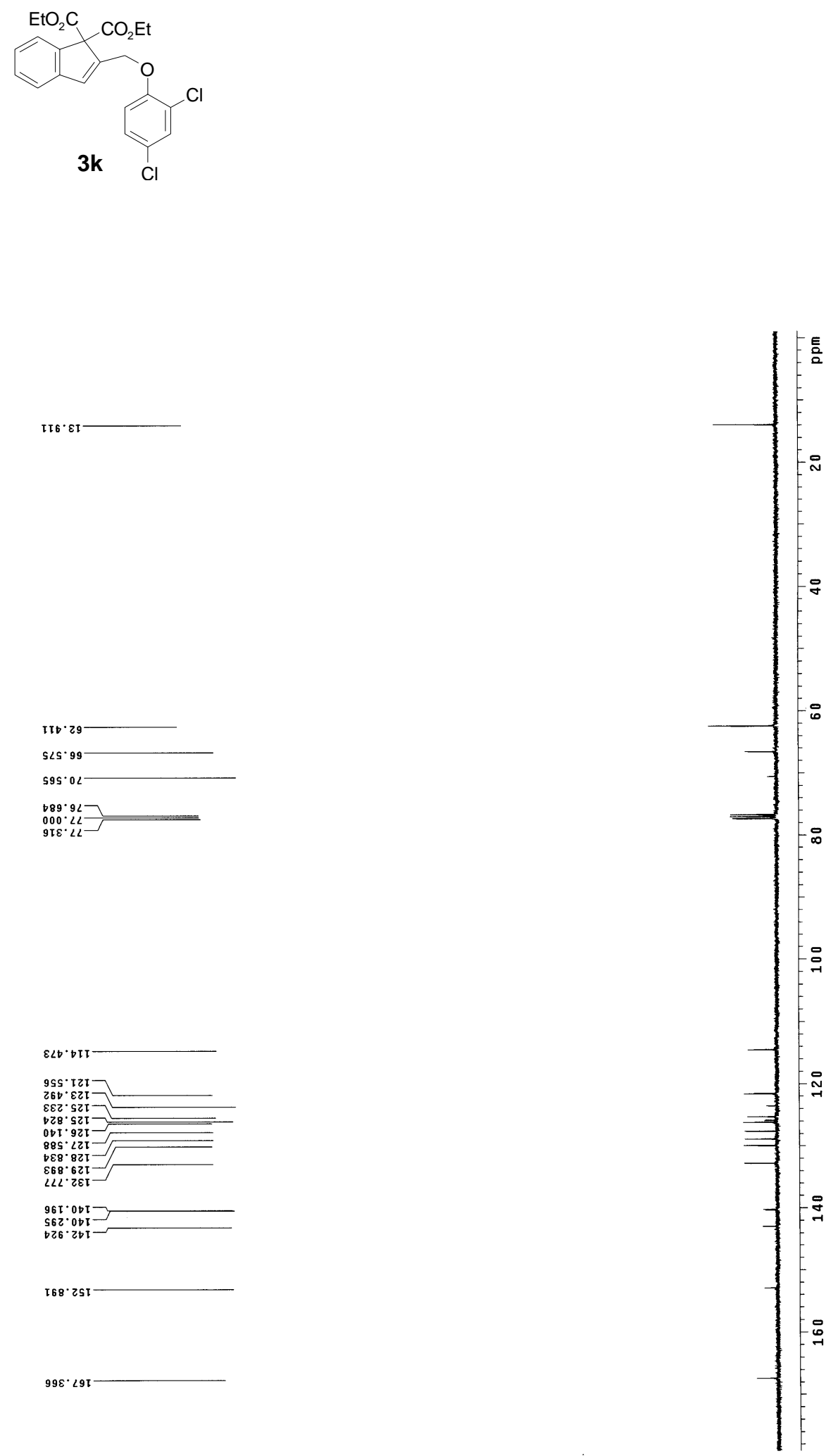

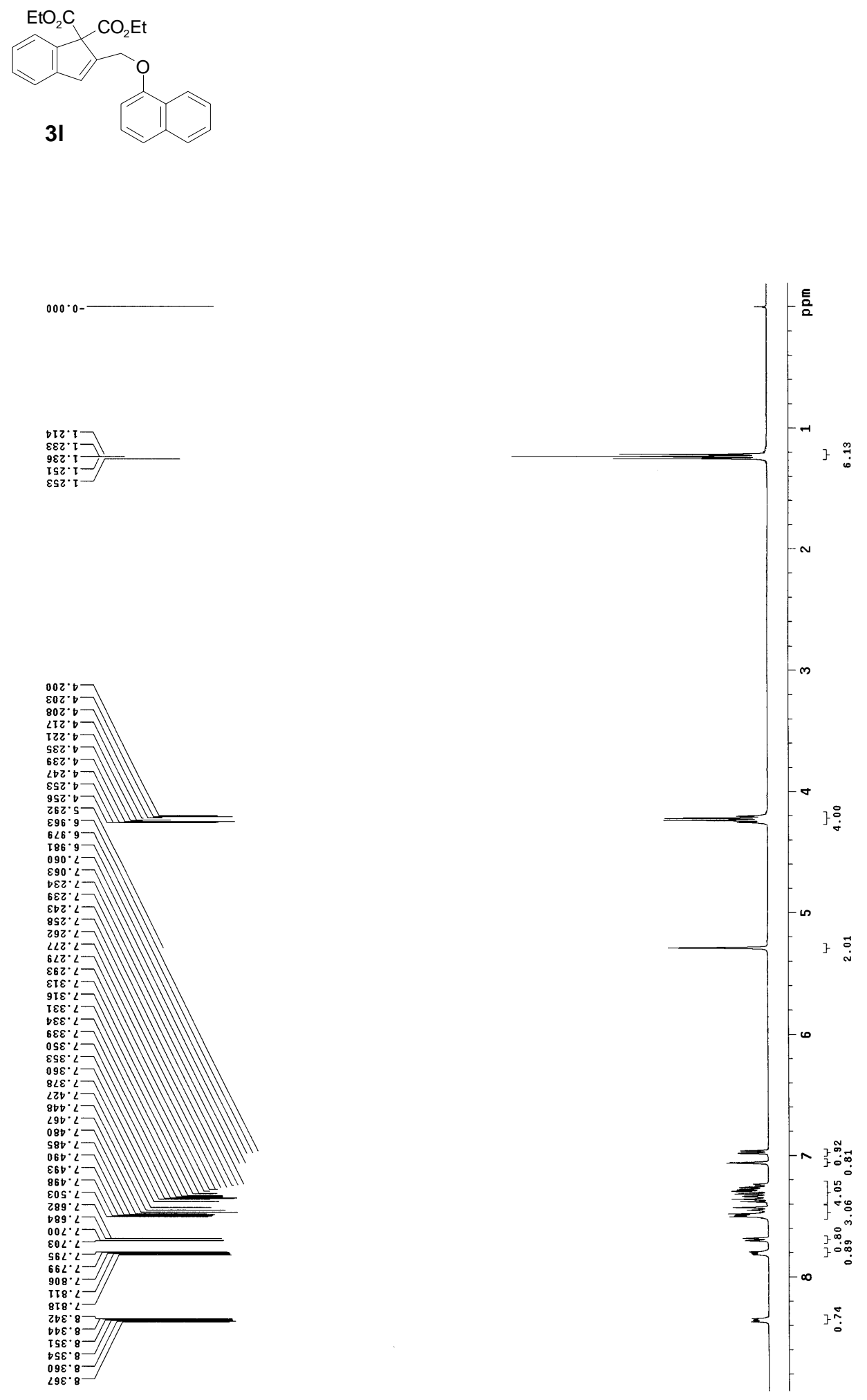


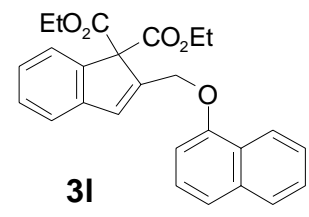

โโ $6 \cdot \varepsilon \tau$

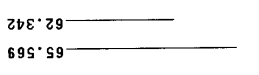

$8<2 \cdot 0<-$

$589^{\circ} 9 L$

${ }_{0} 00 \cdot 2 L=$

$82 \tau \cdot 50 \tau$

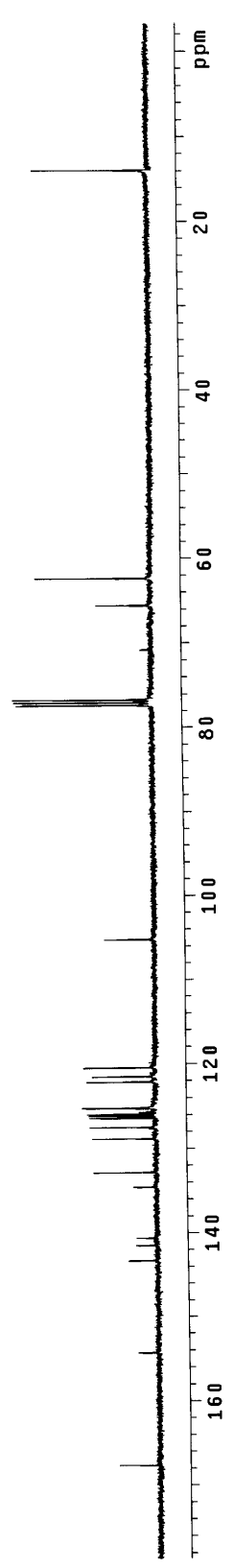

$\angle D O \cdot 02 T$

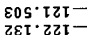

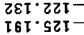

sLZ. sZI

$888 \cdot 521$
901.921

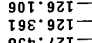

$850^{\circ} \cdot 27 \mathrm{~T}$

SI8. $2 \varepsilon I$

$09 S^{\circ} \forall \varepsilon \tau$

$Z \angle b^{\circ} \tau b \tau$
$b \angle \tau^{\circ} \varepsilon b \tau-$

$80 \varepsilon^{\circ} \sqcup \mathrm{SI}-$

$8 \varepsilon 9^{\circ} \angle 9 \tau$ 

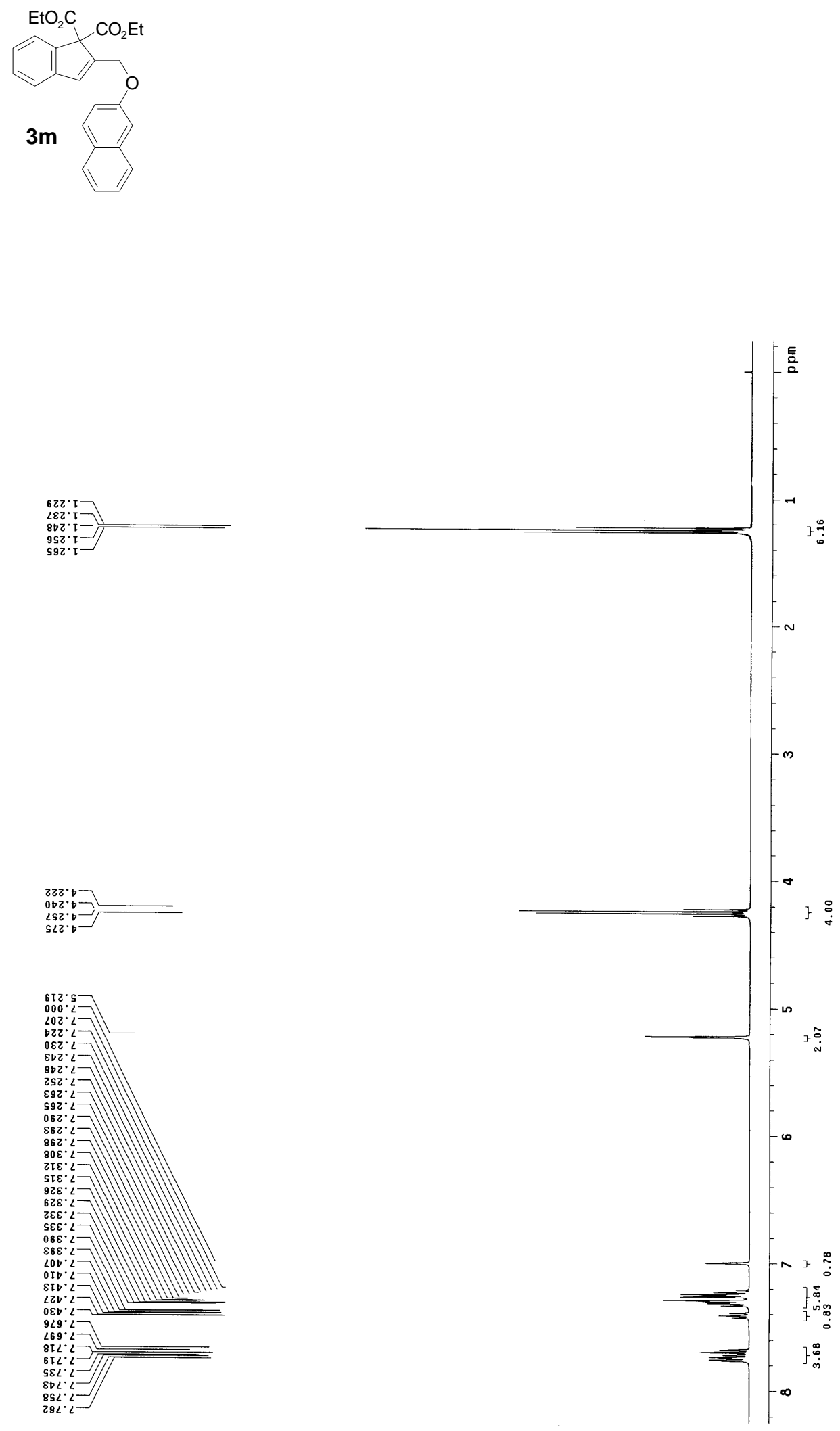


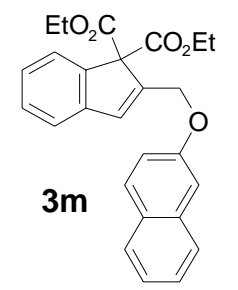

$0 \varepsilon 6 \cdot \varepsilon \tau$
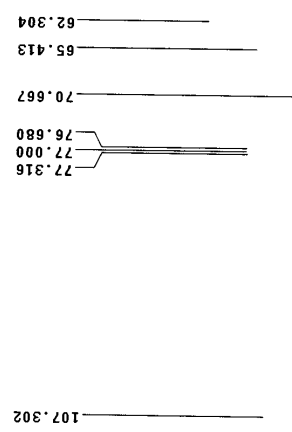

$669.8 \mathrm{II}$

$\mathrm{S9t} \cdot \mathrm{T} / \mathrm{I}$
$\varepsilon \varepsilon 9 \cdot \varepsilon Z \mathrm{I}$

$891 \cdot 5 Z I$
$9 S 0 \cdot 9 Z I-$

$950 \cdot 9 Z I-$
S8Z. $9 Z 1-$

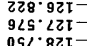

$925 \cdot 22 \mathfrak{r}$

$280.62 \tau-$

$258 \cdot 62 t-$
$808 \cdot 28 I-$

8 IS. $b \varepsilon \mathrm{LI}$

$6 \varepsilon \mathrm{s}^{\circ} \mathrm{0bT}$

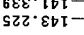

$0 \varepsilon S^{*} \cdot 9 S I-$

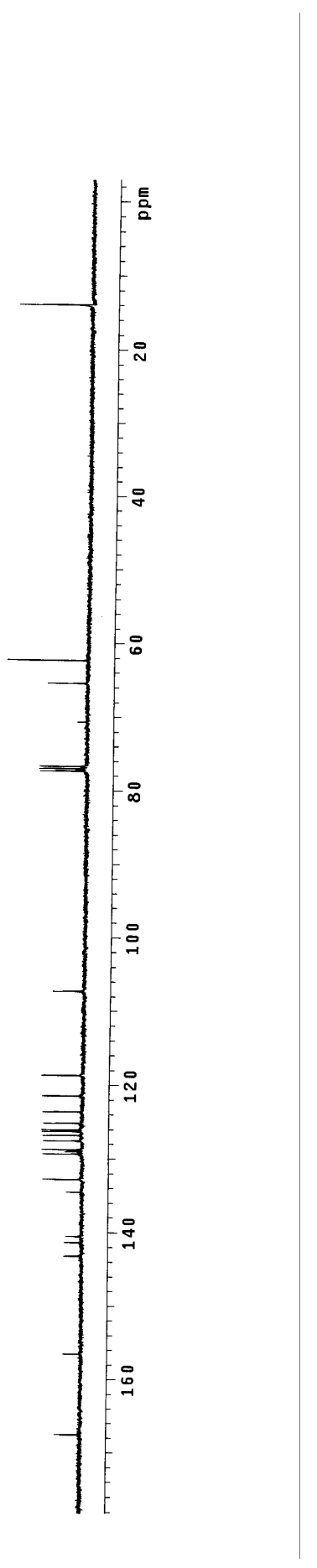

$2 \angle S^{\circ} \angle 9 \tau$ 


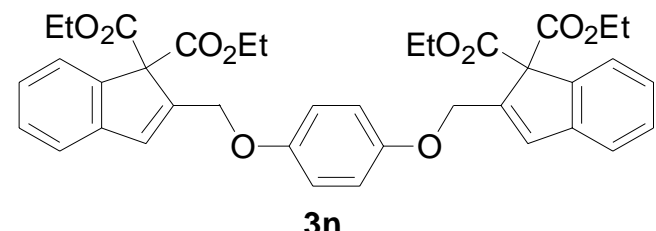

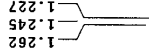

$861 \cdot b-$

原

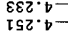

$800 \cdot 5$
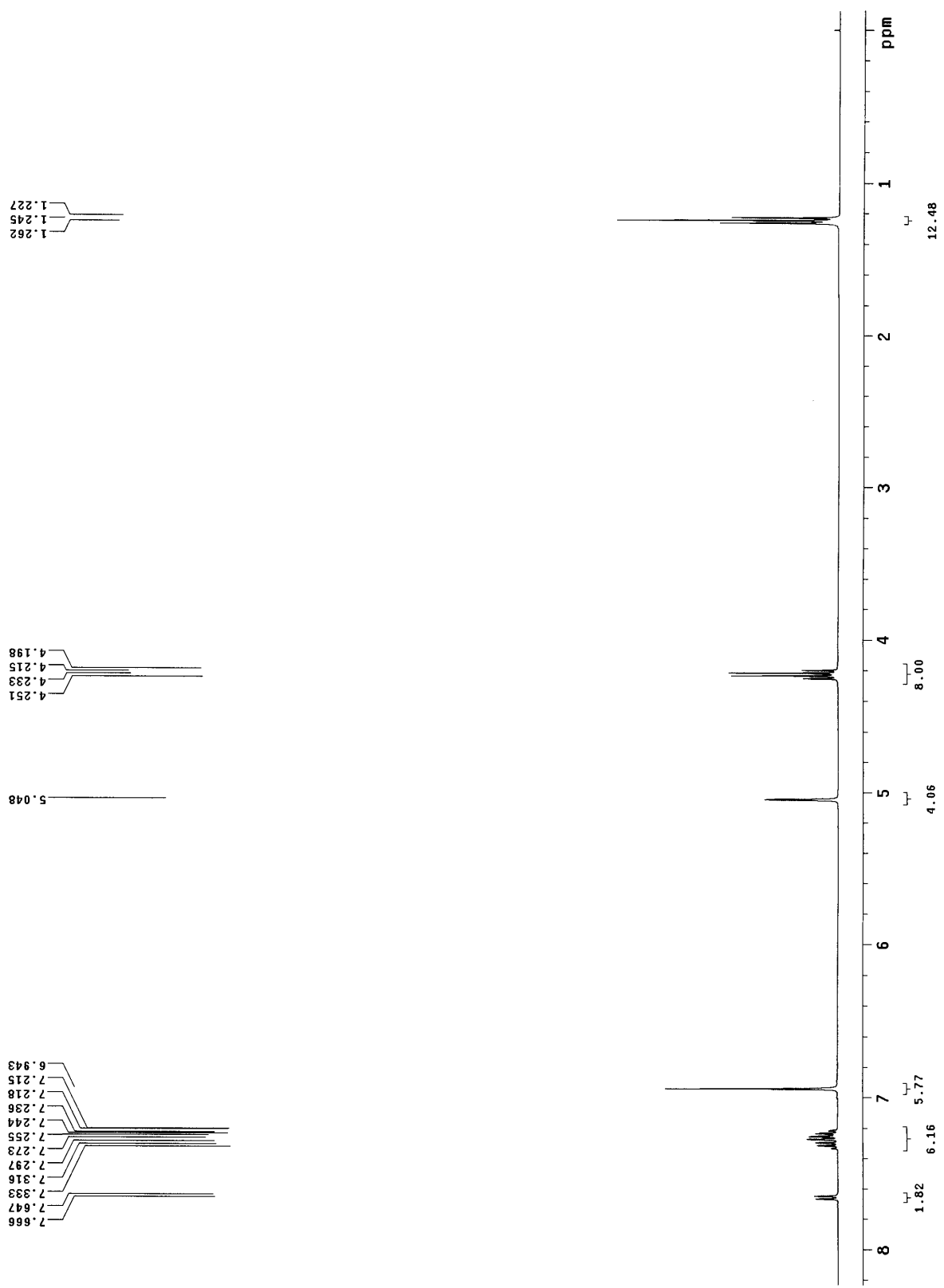

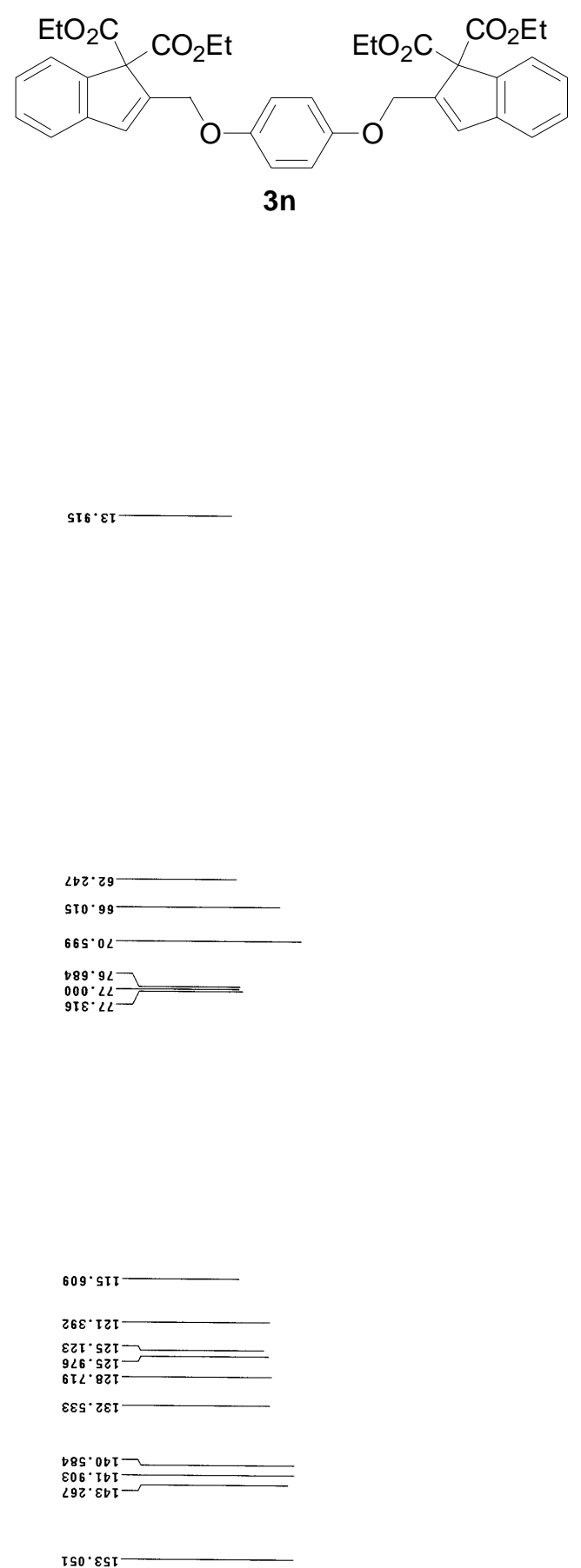

$88 \mathrm{~S}^{\circ} \angle 9 \mathfrak{x}$

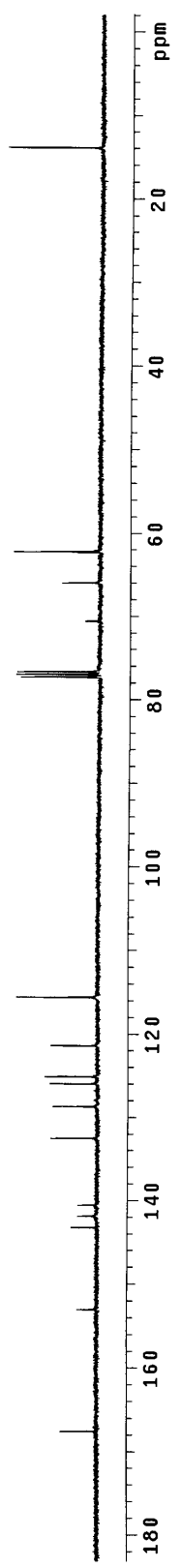




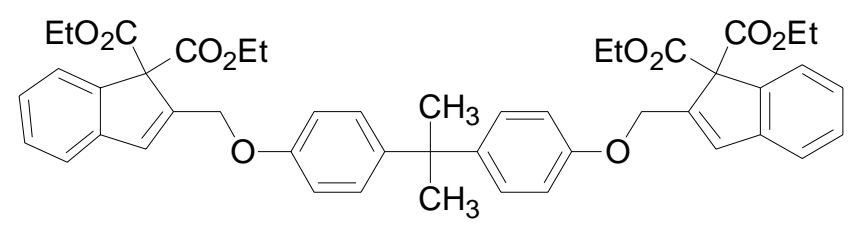

30

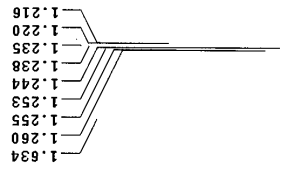

$68 \mathrm{~T} \cdot b$

$86 \tau \cdot b]$

0 iz: $b=$

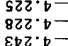

$\varepsilon \rightarrow z$
$s \rightarrow b$

$9 \angle 0^{\circ} \mathrm{s}$
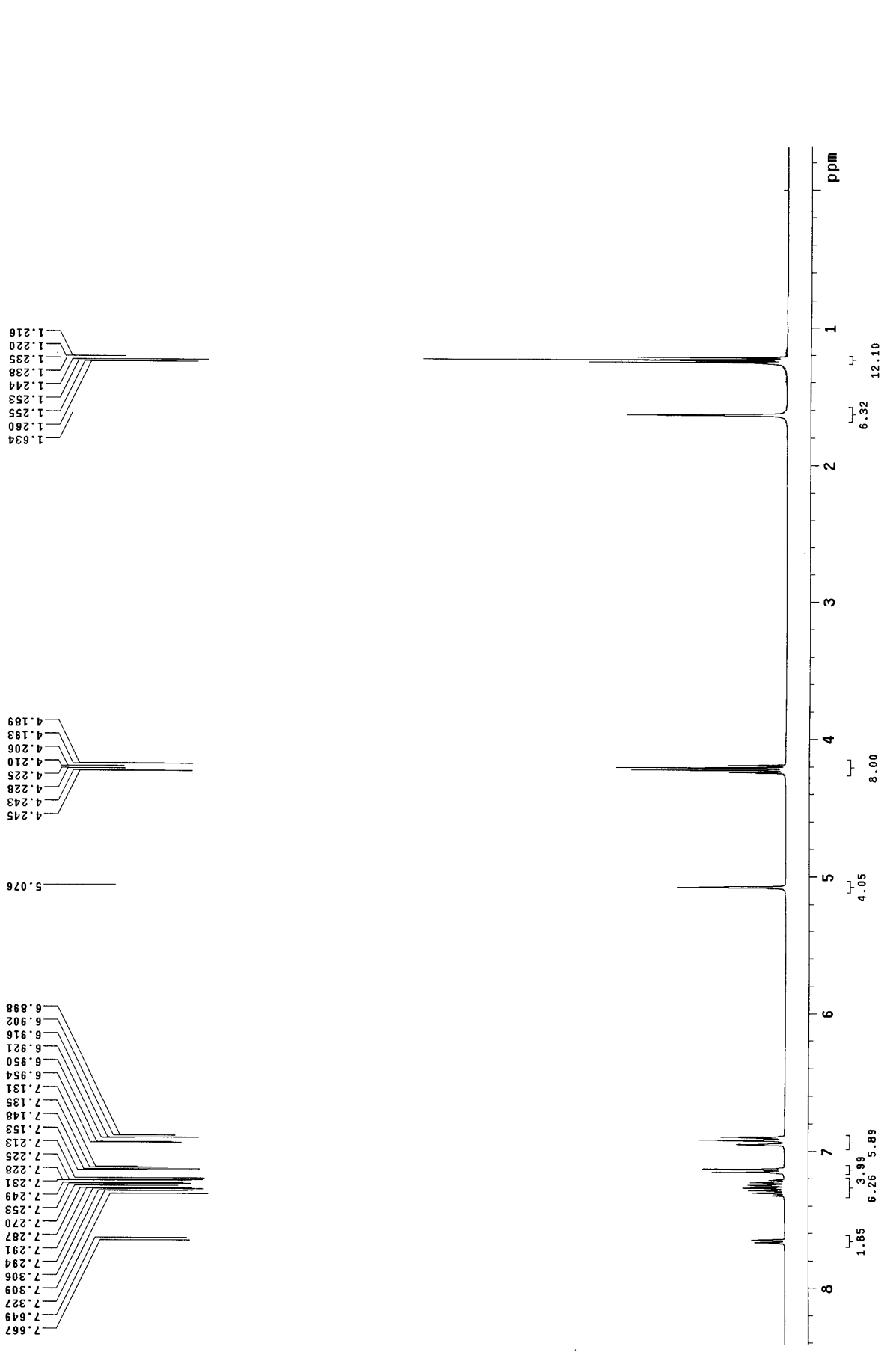


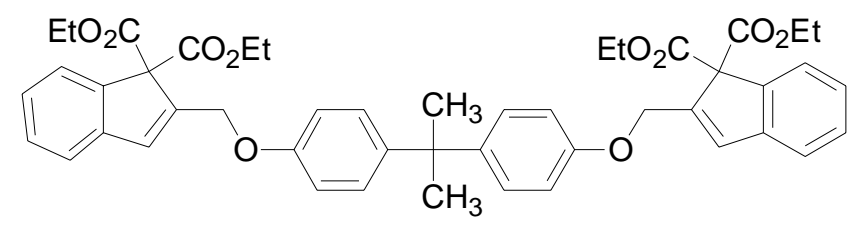

30

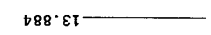

$266 \cdot 0 \varepsilon$

$D \varepsilon 9^{*}$ โ

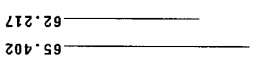

เ89.9L

$000^{\circ}<2$

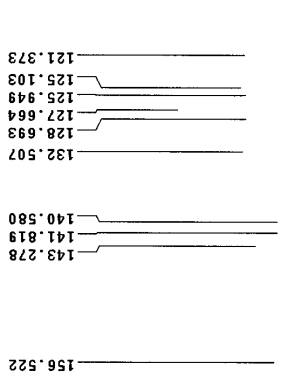

$665 \cdot \angle 9 T-$

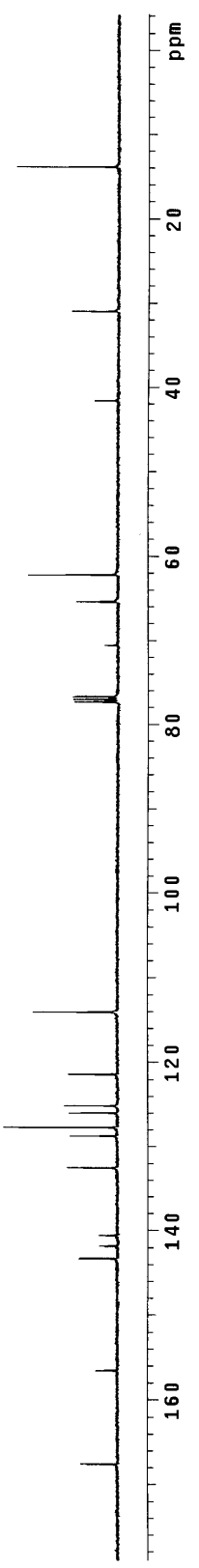



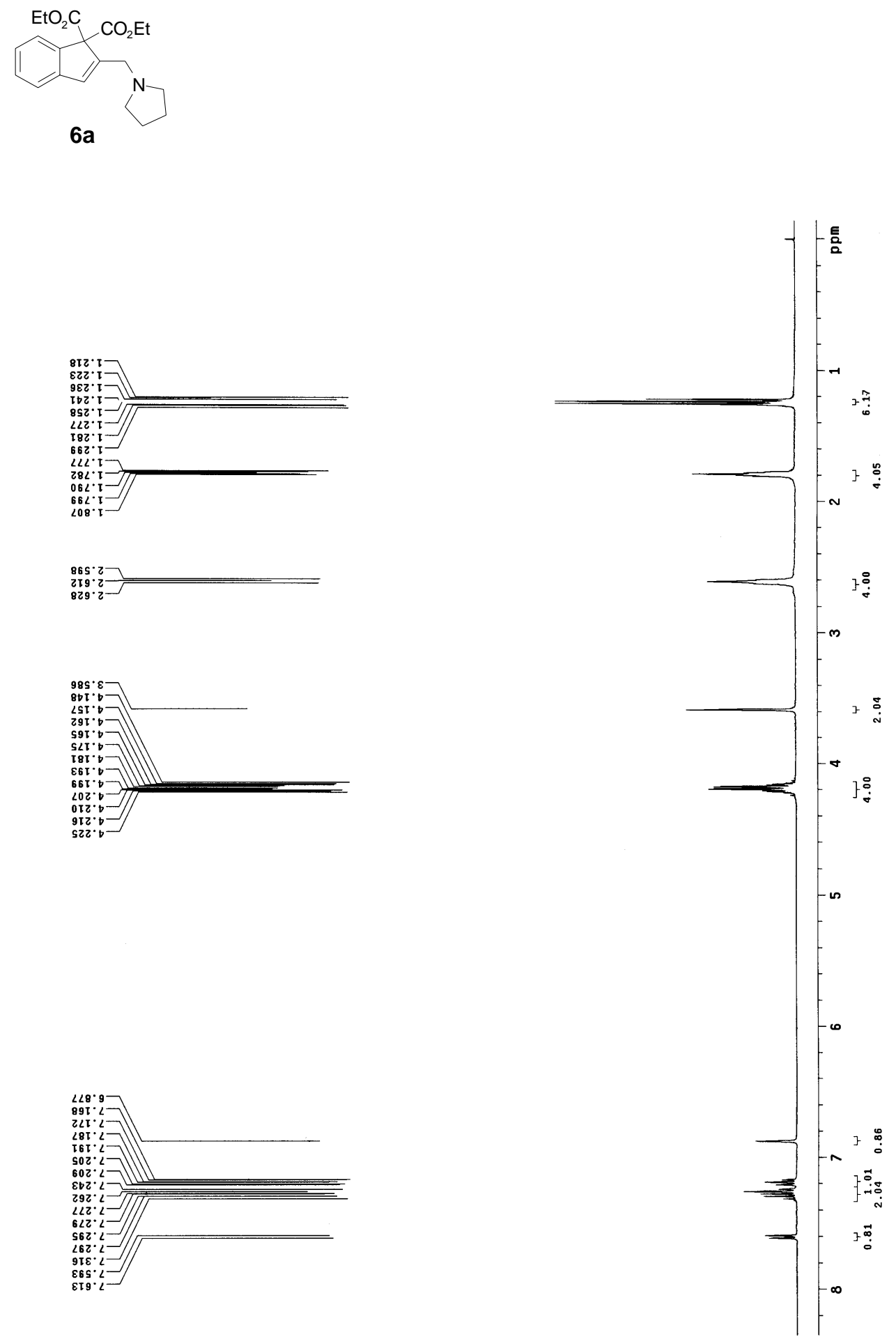


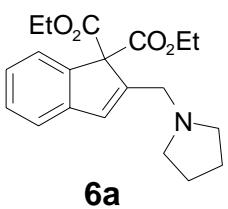

$\varepsilon 06 \cdot \varepsilon \tau$

$889 \cdot \varepsilon z$

$6<\varepsilon \cdot b S-$
$\varepsilon 6 D \cdot b S-$

$\angle 08 \cdot 19$

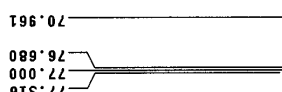

9T $8 . \angle L$

$\angle 98.025$

$6 \angle 8 \cdot 621=$

$59 \mathrm{D} \cdot \mathrm{SZT}$

กนร' $22 \mathrm{I}$

$\angle 68.00 \tau$

$\begin{array}{ll}800.061 \\ 6<1 & 501\end{array}$

$060 \cdot 89 \tau$

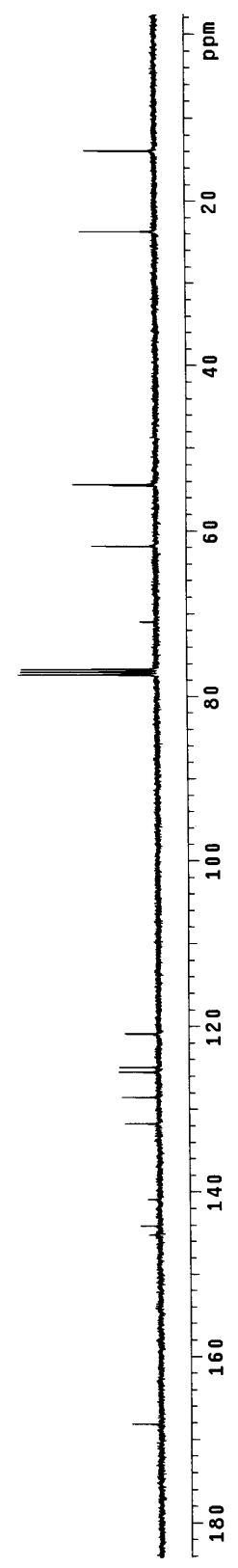



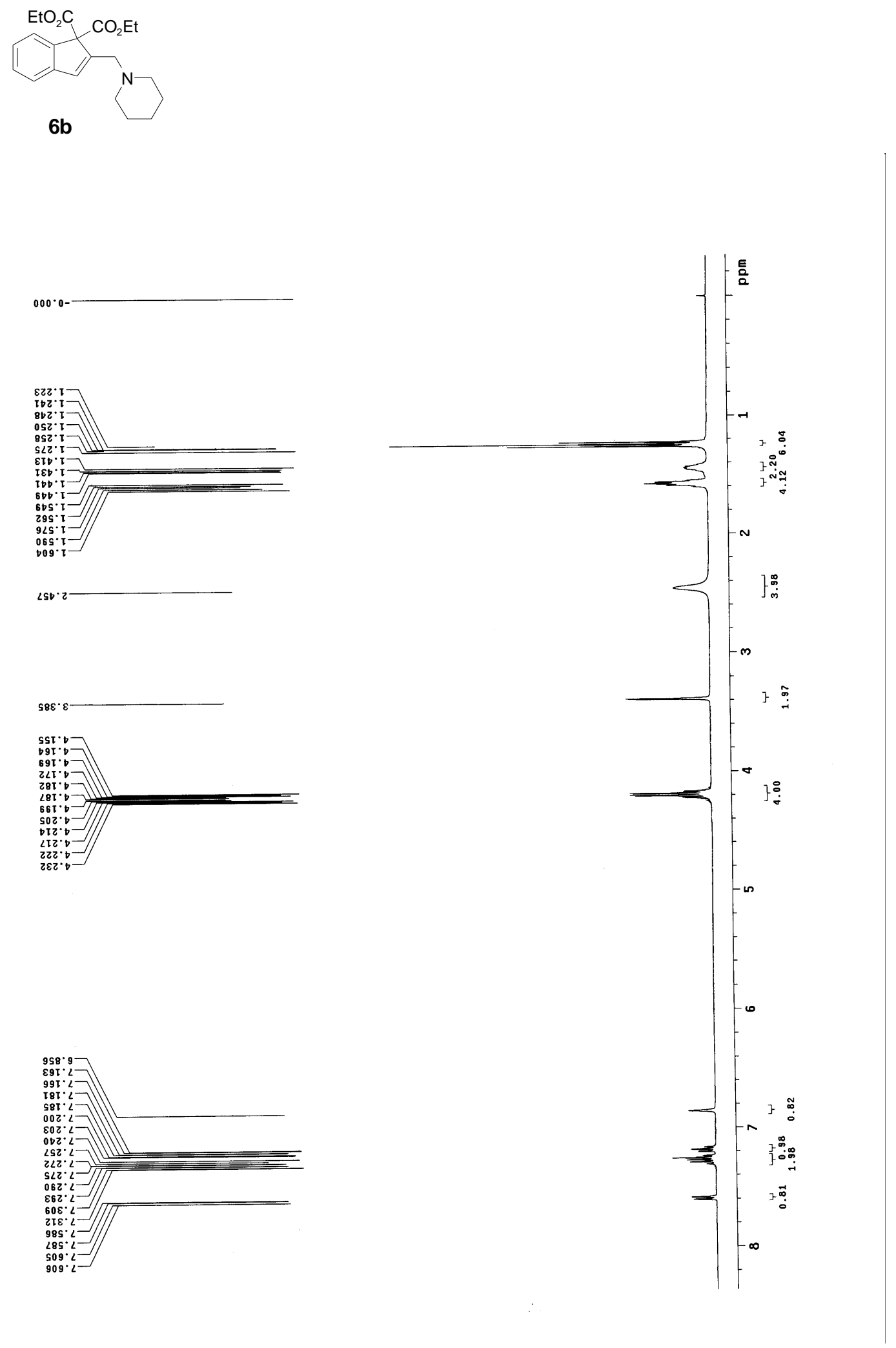


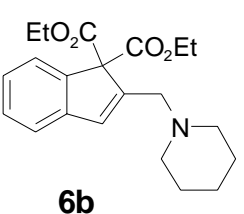

Sโ6. $\varepsilon \tau$

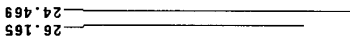

600.25

s\&8. 29

$968 \cdot 02$

689.92

${ }_{020}^{600} \cdot 2 L$

SS8.0टा

$\angle 98 . b 2 t=$

$8 \not b \cdot .5 z T$

$9 \angle 0^{\circ} 281$

9st' tot

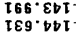

$060.89 \tau$

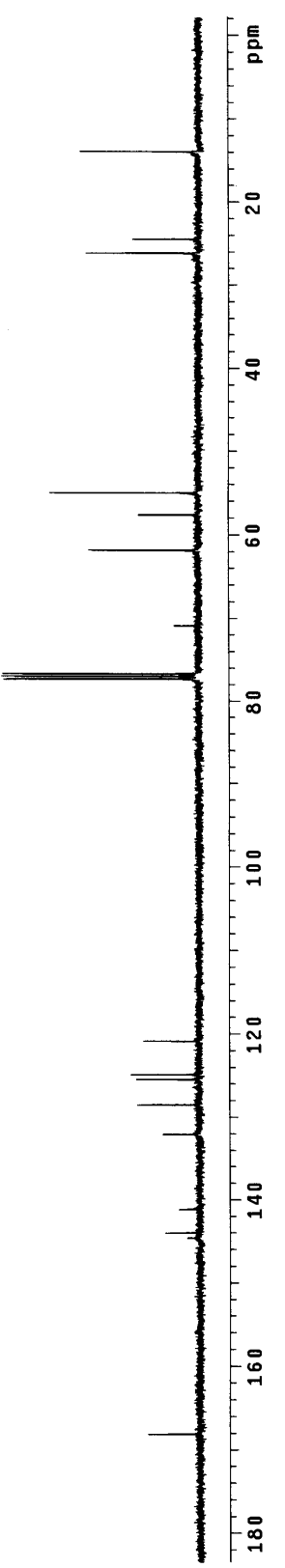



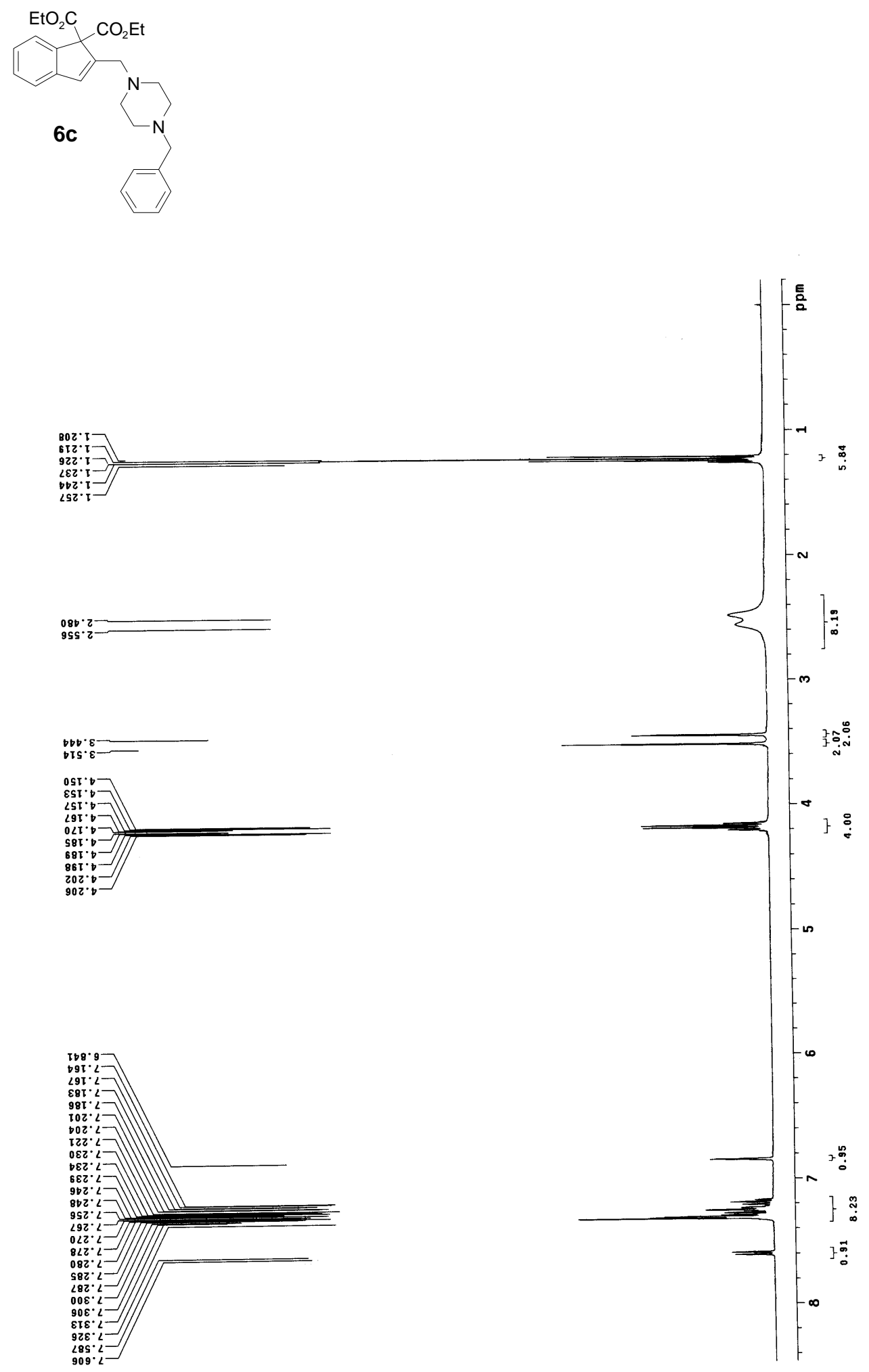


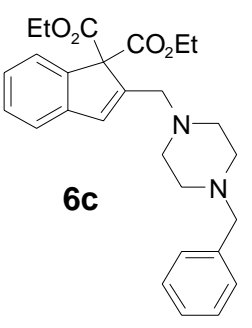

$268 \cdot \varepsilon 1$
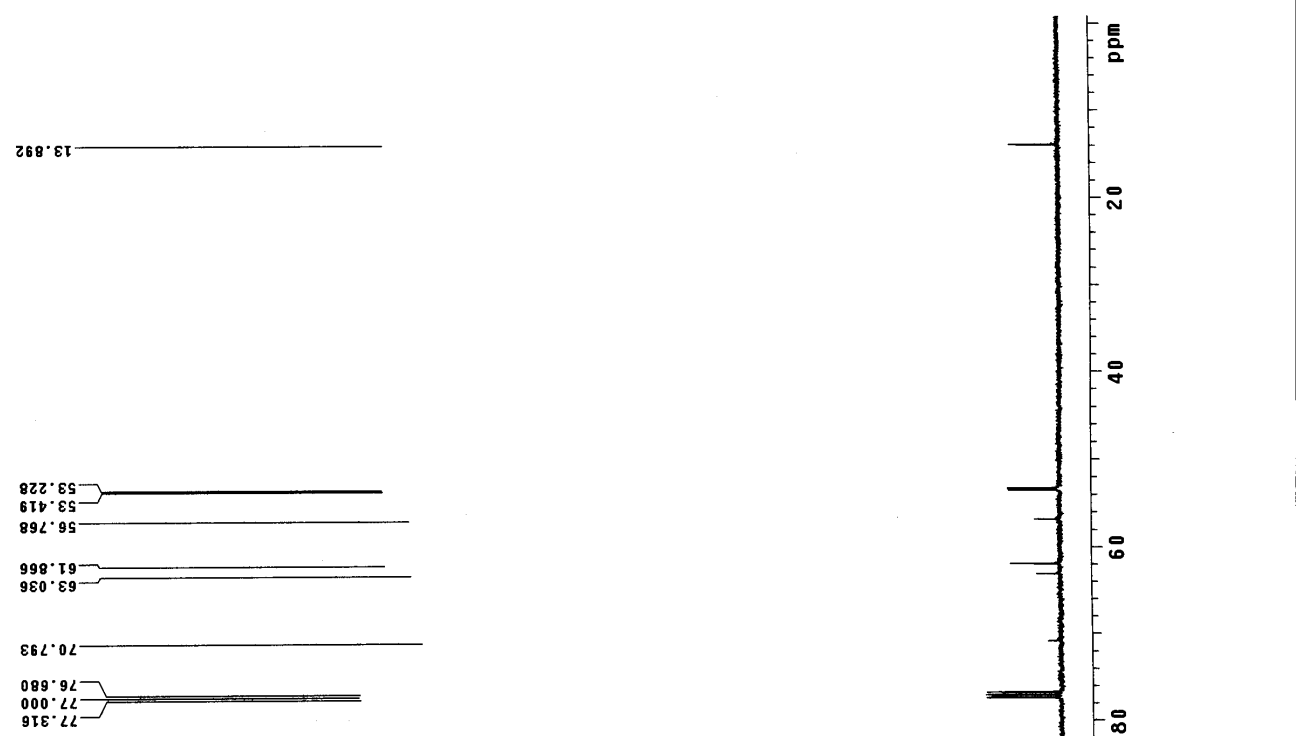

$0<8 \cdot 0217$

SL8.bZI-

626.921

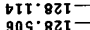

909.825

$8 \mathrm{SI} \cdot 62 \mathrm{I}$
$\angle D E \cdot 2 \varepsilon \mathrm{I}$

$0 \varepsilon \tau \cdot 8 \varepsilon \tau-$

ดII. IOI

$\angle 90^{\circ} \circ 6 \mathrm{DT}$

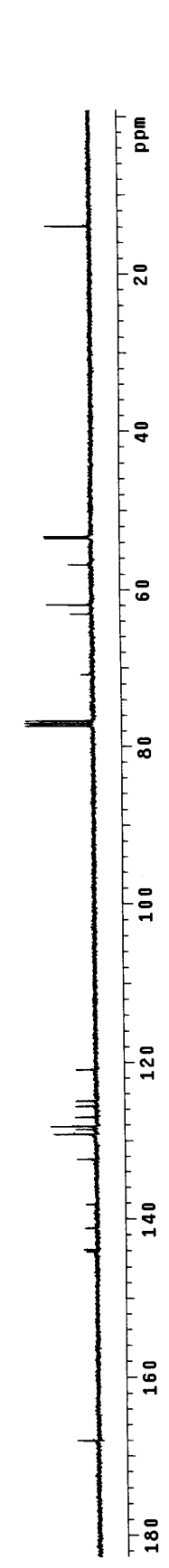




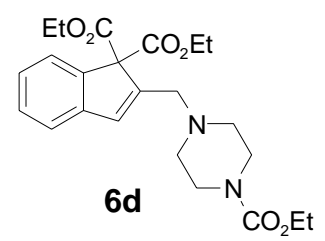

$000.0-$

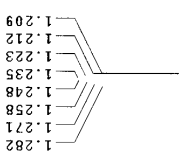

$266 \cdot 2-$

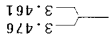

Tot.

$50 T \cdot b$
$52 T \cdot b$

$80 T \cdot \circ-$
$291 \cdot 0-$

$\varepsilon<1 \cdot b=$

S8. 6 -

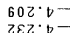
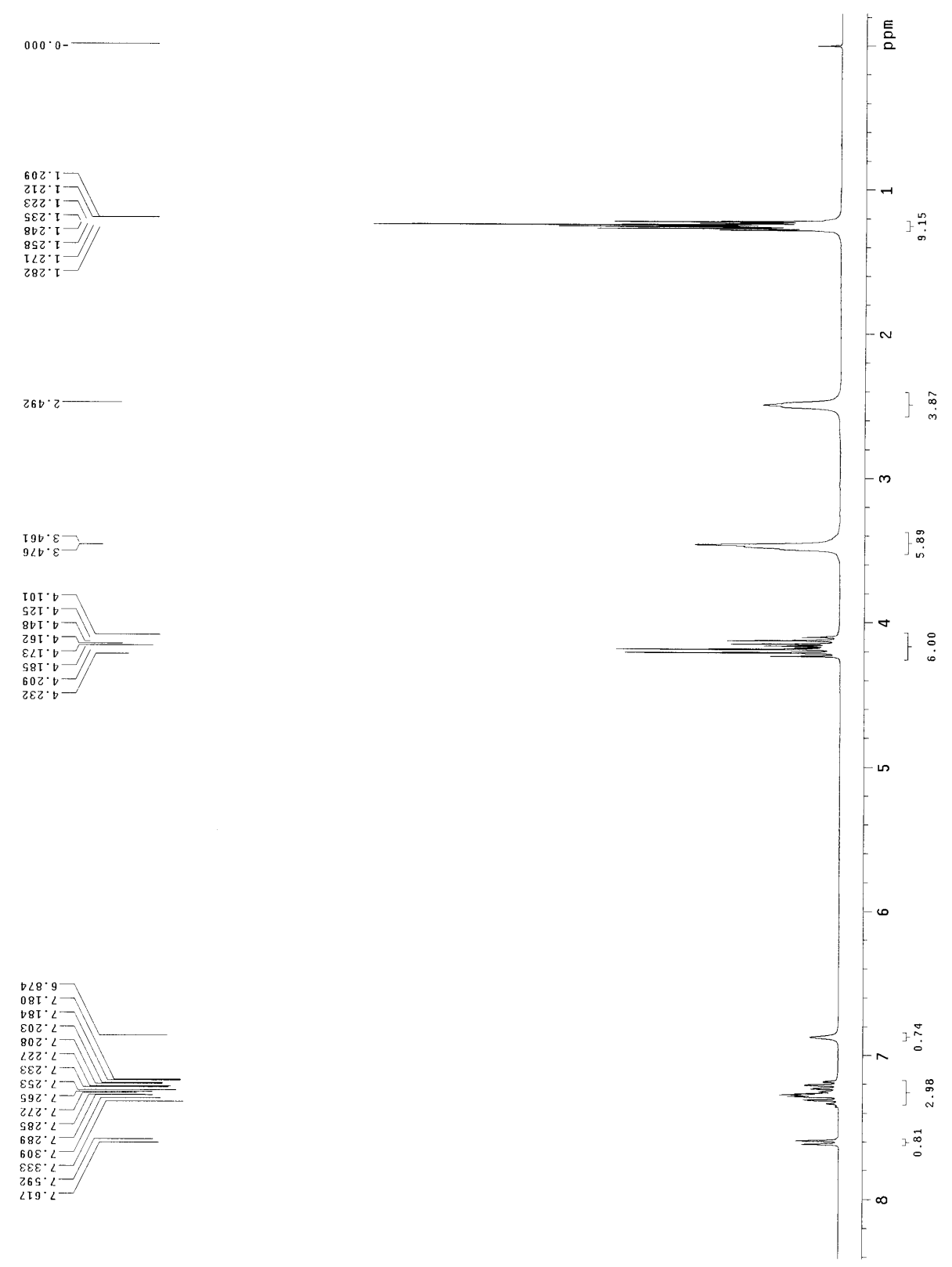

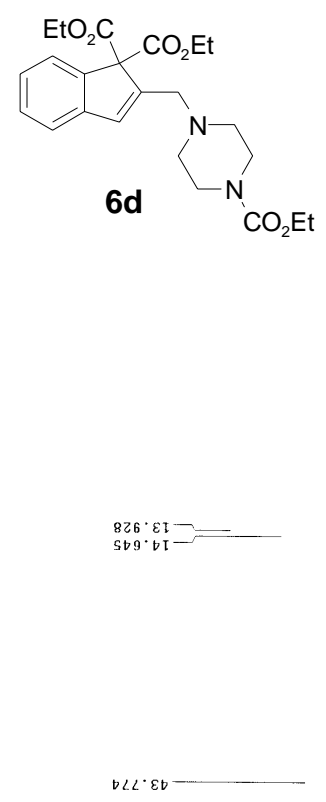

$880 \cdot \varepsilon \mathrm{s}$

โ 88.95

$\angle 52 \cdot 19=$
$896.19=$

$2 \pi \angle 0<-$

$9.5 \cdot 92=$
$000 \cdot 2 L-$

$t 2 t \cdot 2 L$

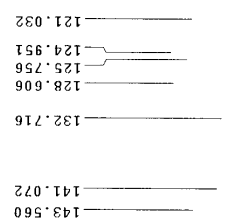

$\angle E S \cdot S S I$

6 I6. $\angle 91-$

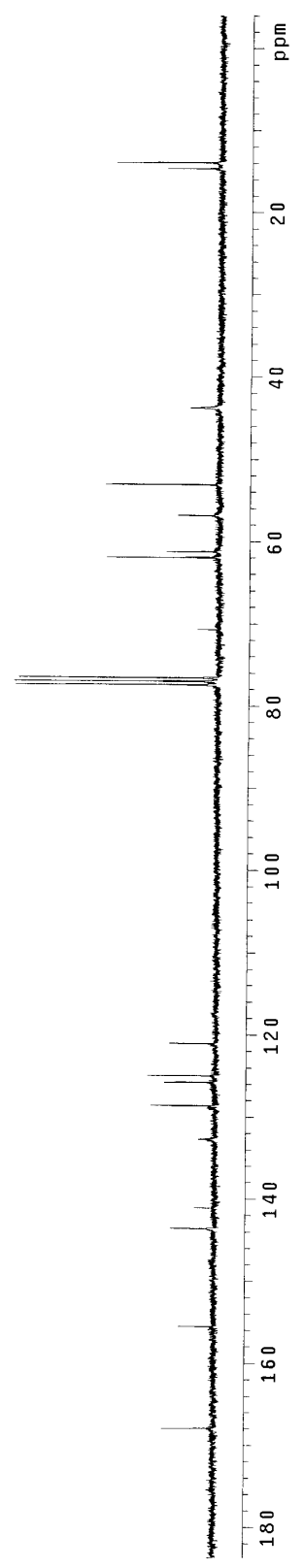



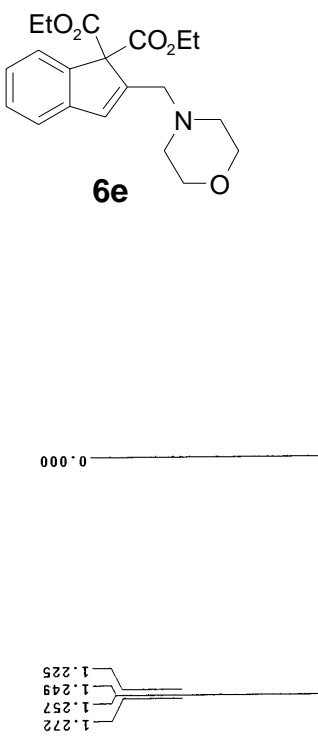

SIS:
82S:
-
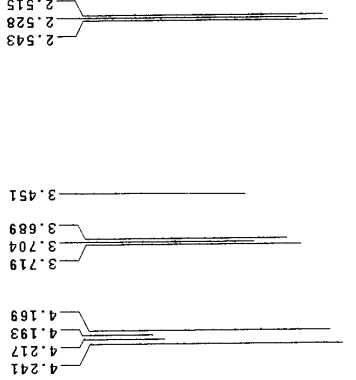

$\varepsilon 6 \tau \cdot b$
$2 \tau z \cdot b$
$\tau b z$

98.9-

$\varepsilon<\mathrm{t} \cdot<7$

$821 \cdot 2$
$865 \cdot 2-2$

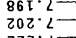

222 2 . 27

(282.2

$\varepsilon 92 \cdot 2=$

$0 .<-$
082

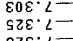

$828 \cdot 2$

565.:

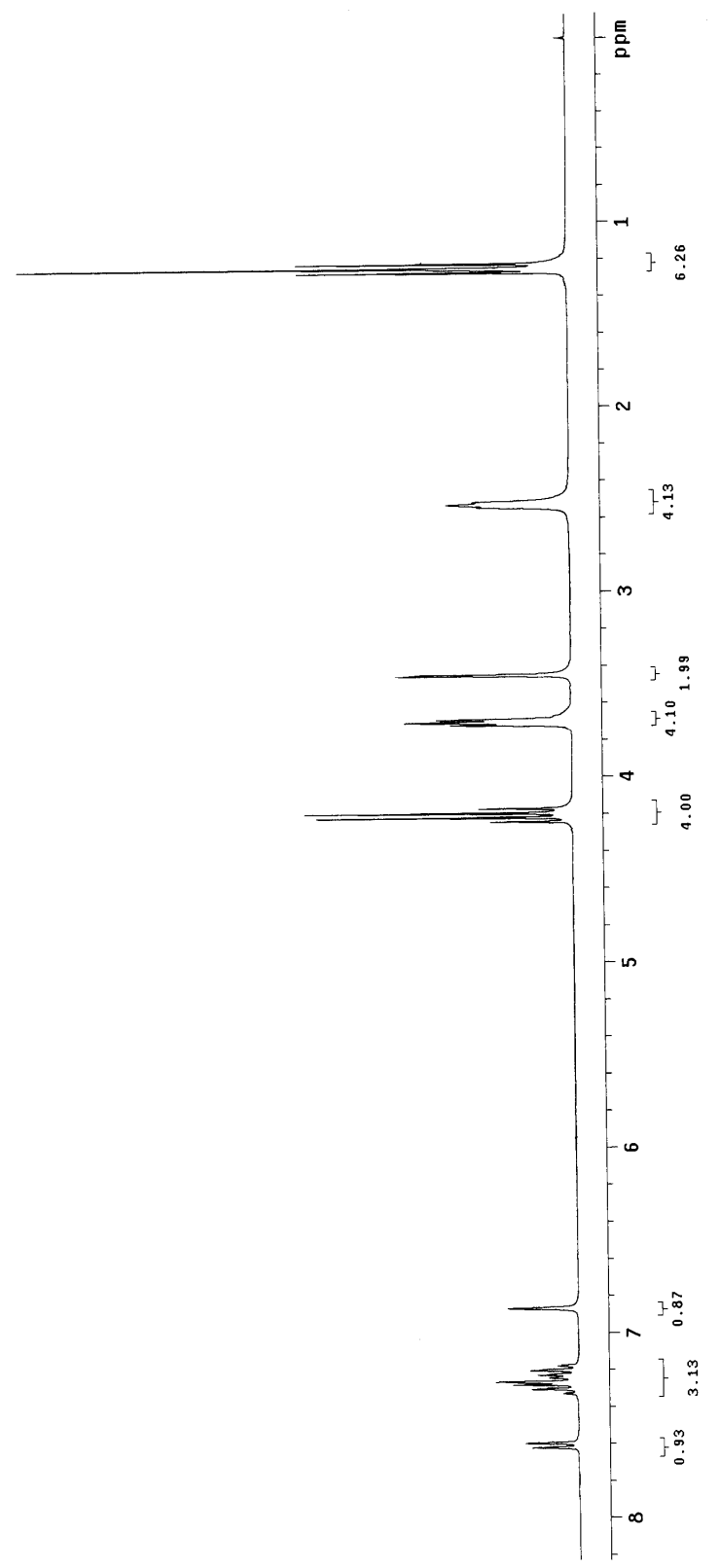




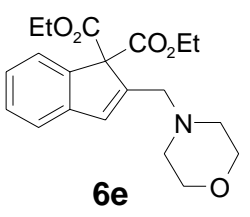

$\tau 06 \cdot \varepsilon \mathfrak{-}$
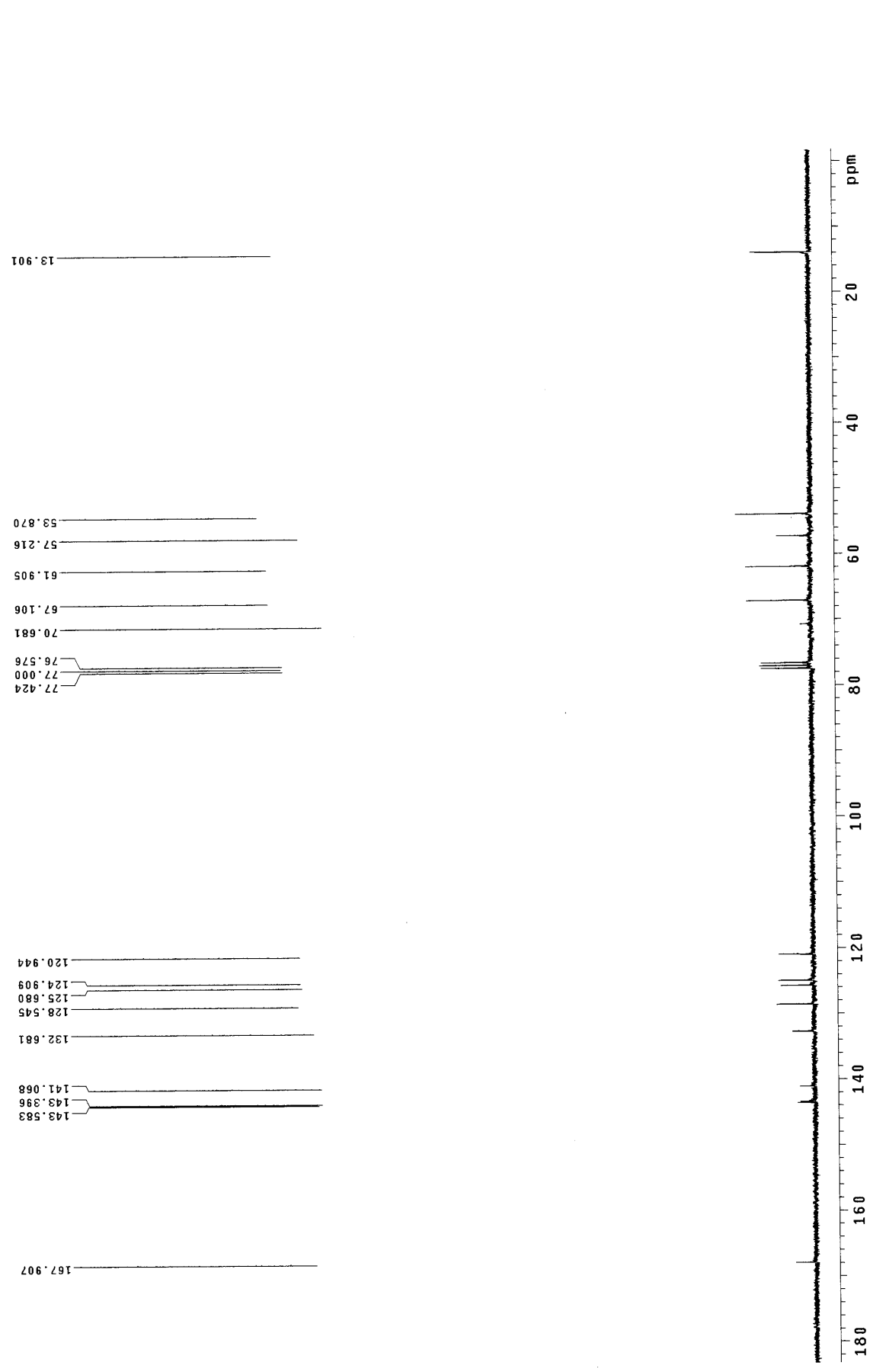

$\varepsilon 8 \mathrm{~s} \cdot \mathrm{\varepsilon bt}$

$\angle 06 . \angle 9 \tau+\ldots$ 


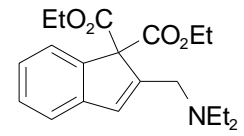

$6 f$
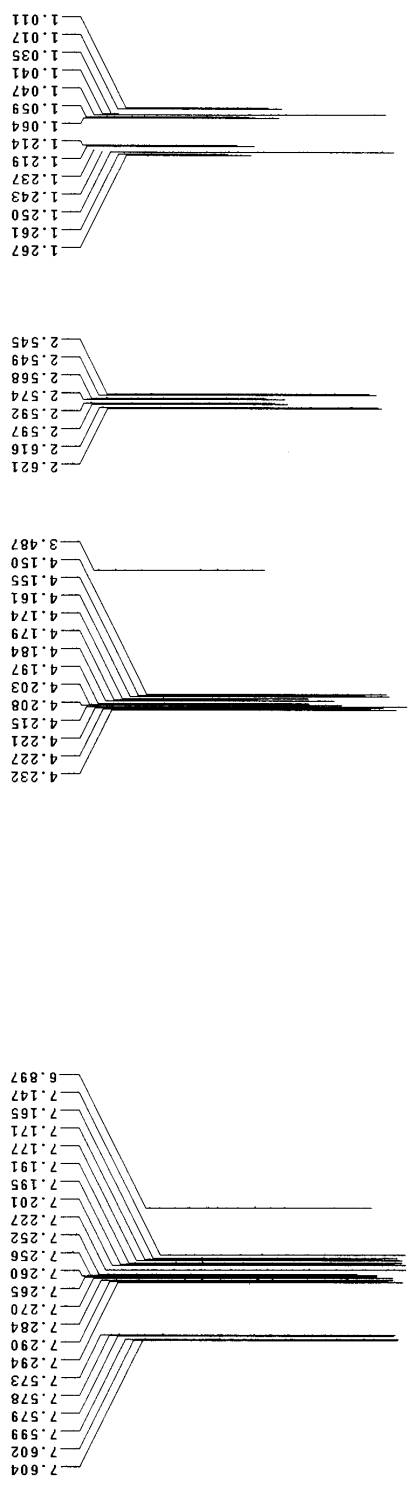

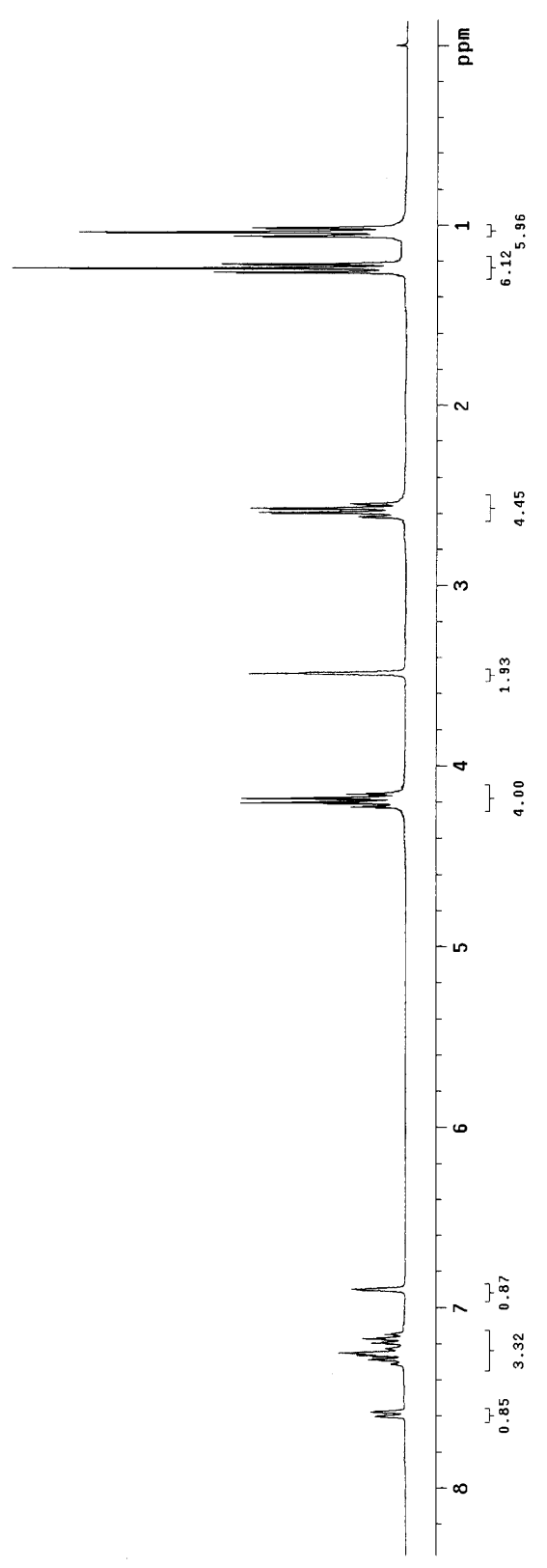




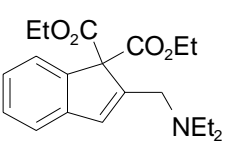

$6 f$
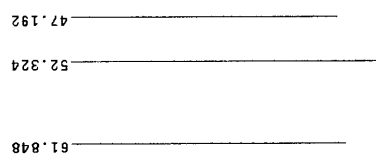

$90<\cdot 0<-$

$\varepsilon \angle S \cdot 9 L>$
$000 \cdot 2 L$
$0 Z D \cdot L L$

$962.02 I$

$\rightarrow 62 \cdot 52 \mathrm{~T}$

$660^{\circ} 8 Z I$
$509^{\circ} \tau \varepsilon I$

$\tau 60^{\circ} \tau t \tau$
$\angle E 0^{\circ} \circ t \tau$

$\angle E 0^{\circ} D D I$
$96 I \cdot 9 D I-$

$S S T \cdot 89 \tau$

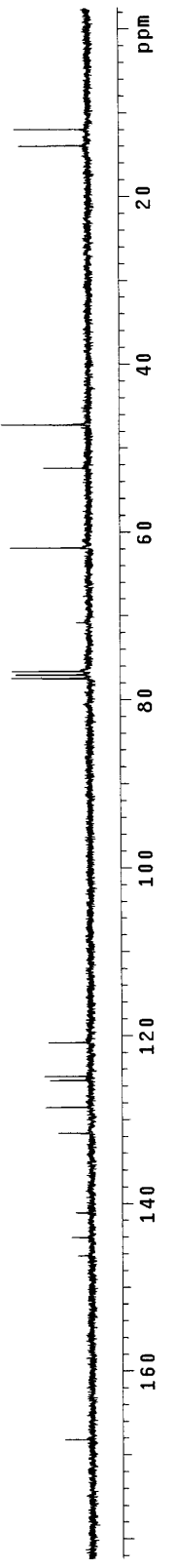



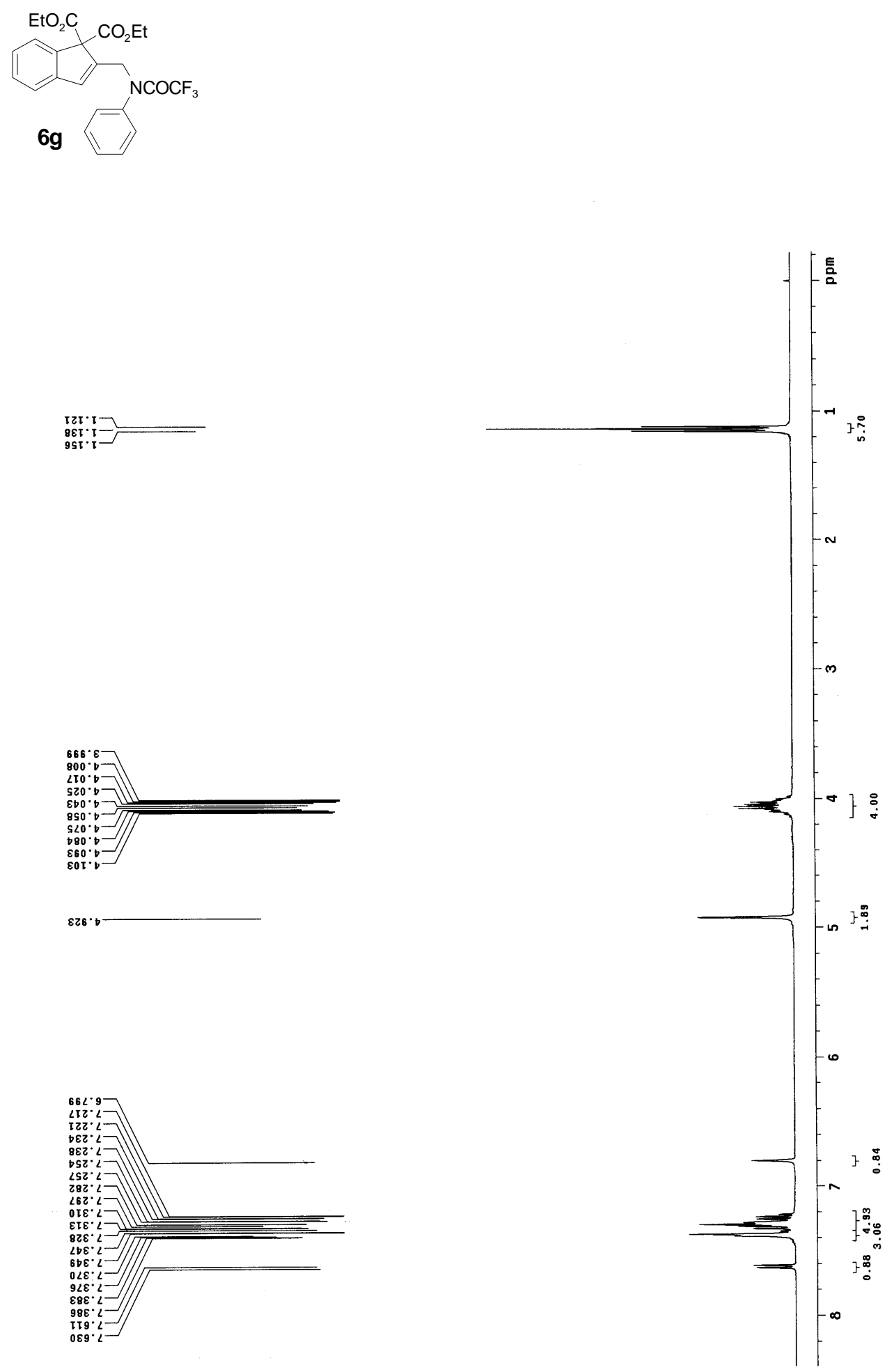


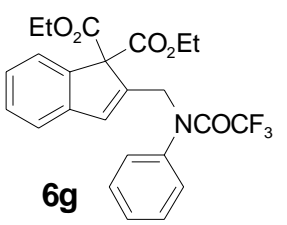

$9 \varepsilon\llcorner\cdot \varepsilon \tau$

t08.0s

$\angle b z \cdot z 9$

$\angle 2 \varepsilon^{\prime} \cdot \iota$

689.927

${ }_{9 \tau}^{000}<\angle L$
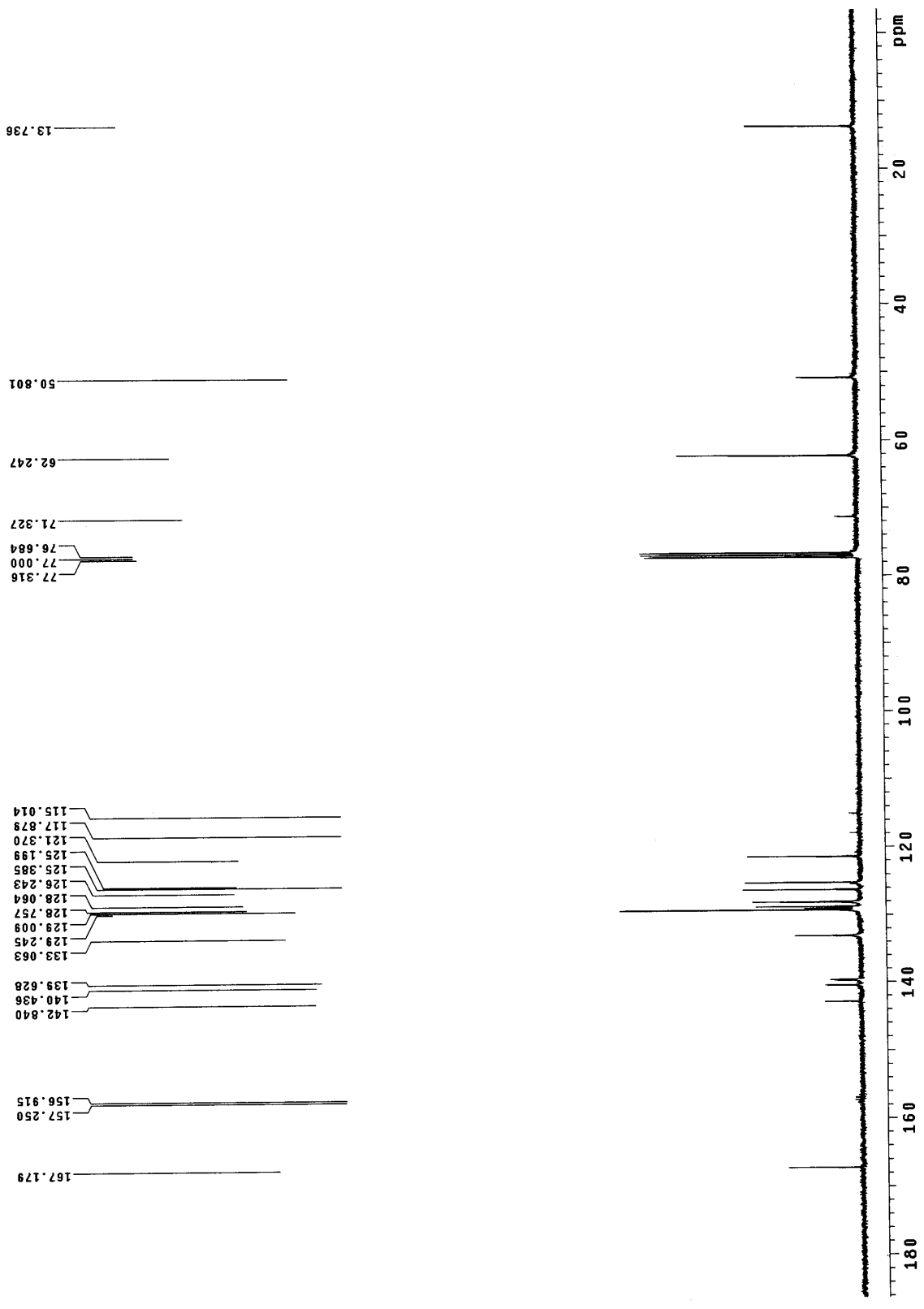